Portland State University

PDXScholar

$6-2008$

\title{
Sustainability From the Perspectives of Indigenous Leaders in the Bioregion Defined by the Pacific Salmon Runs of North America
}

David Edward Hall

Portland State University

Follow this and additional works at: https://pdxscholar.library.pdx.edu/open_access_etds

Part of the Indigenous Studies Commons, and the Social Psychology Commons Let us know how access to this document benefits you.

Recommended Citation

Hall, David Edward, "Sustainability From the Perspectives of Indigenous Leaders in the Bioregion Defined by the Pacific Salmon Runs of North America" (2008). Dissertations and Theses. Paper 2569.

https://doi.org/10.15760/etd.2566

This Dissertation is brought to you for free and open access. It has been accepted for inclusion in Dissertations and Theses by an authorized administrator of PDXScholar. Please contact us if we can make this document more accessible: pdxscholar@pdx.edu. 


\title{
SUSTAINABILITY FROM THE PERSPECTIVES OF \\ INDIGENOUS LEADERS IN THE BIOREGION DEFINED BY \\ THE PACIFIC SALMON RUNS OF NORTH AMERICA
}

\author{
by \\ DAVID EDWARD HALL
}

A dissertation submitted in partial fulfillment of the requirements for the degree of

\author{
DOCTOR OF PHILOSOPHY \\ in \\ SYSTEMS SCIENCE: PSYCHOLOGY
}

Portland State University

2008 


\begin{abstract}
An abstract of the dissertation of David Edward Hall for the Doctor of Philosophy in Systems Science: Psychology presented June 10, 2008.

Title: Sustainability from the Perspectives of Indigenous Leaders in the Bioregion Defined by the Pacific Salmon Runs of North America

Extensive research suggests that the collective behavior of humanity is on an unsustainable path. As the evidence mounts and more people awaken to this reality, increased attention is being dedicated to the pursuit of answers for a just and sustainable future. This dissertation grew from the premise that effectively moving towards sustainability requires change at all levels of the dominant Western culture, including deeply held worldviews. The worldviews of many indigenous cultures offer alternative values and beliefs that can contribute to addressing the root causes of problems related to sustainability. In the bioregion defined by the Pacific Salmon runs of North America there is a rich heritage and modern day presence of diverse indigenous cultures. In-depth semi-structured interviews were conducted with 13 indigenous leaders from within this bioregion to explore their mental models of sustainability. These interviews followed a general structure that covered: (a) the personal background and community affiliation of each interviewee; (b) the meaning of the concept of sustainability from their perspective; (c) visions of a sustainable future for their communities; and, (d) how to achieve such a future.

A content analysis of the interviews was conducted and summarized into a narrative organized to correspond with the general interview structure. A process of testimonial validity established that most participants found the narrative to be an accurate representation of their perspectives. Participant feedback led to several phrasing changes and other identified issues are discussed, including one participant's critique of the narrative's use of a first-person plural voice. Major themes from the interviews include the role of the human being as caretaker actively participating in the web of life, the importance of simultaneously restoring culture and ecology due to their interdependence, the need to educate and build awareness, and the importance of cooperation. Understanding who we are as a living species, including our profound connection with nature, along with a holistic and intergenerational perspective are suggested as prerequisite for balancing and aligning human modes of being with the larger patterns of life. The closing discussion addresses the importance of social action and going beyond a conceptual understanding to an embodiment of sustainability.
\end{abstract}


Dedicated to past, present, and future citizens of Salmon Nation 


\section{ACKNOWLEDGEMENTS}

This project would not have been possible without the support and inspiration of Mother Earth, and many people there within. Much gratitude is felt for each of the below individuals for their respective contributions that helped make this project what it is.

My heartfelt appreciation to each of the project participants: Antone Minthorn (Cayuse), Carol Craig (Yakama), Dennis Martinez (O'odham / Anglo / Chicano), Guujaaw (Haida), Jeannette Armstrong (Syilx), Joeneal Hicks (Ahtna), Kathryn Martin (Ahtna), Larry Merculieff (Aleut), Nicholas Jackson (Ahtna), Nichole Maher (Tlingit), Roberta Conner (Cayuse), Shawn Yanity (Stillaguamish), Wilson Justin (Althsetnay). The knowledge and perspective as shared by each of these individuals is the true source of the value delivered by this project. Further thanks to their assistants and co-workers who helped with arrangements and logistics, including April Davis, Donita Sue Fry, Feather Huesties, Gloria Stickwan, Helen Morrison, and Suezan Pierce.

Thanks to my committee, Dr. Jan Haaken, The Honorable Elizabeth Furse, Dr. Eric Mankowski, Dr. Wayne Wakeland, and Dr. Virginia Butler, for humbly fulfilling their duties as guardians of the degree, for their direction and guidance, for challenging me to confront my assumptions, and for encouraging me to produce high quality work.

Much appreciation to all of the undergraduate research assistants who contributing their time, effort, skills and intellect to the project, including Michelle Emery, Brianna Finney, Derek Valldejuli-Gardner, Price McCloud Johnson, Kelly Henry, Linda Rand, and Jenessa Roberti.

Many thanks to Craig Jacobson and Elizabeth Woody of Ecotrust, who helped with project development and support at various stages. Thanks to Dr. Keith James who also aided in the early conception and development of the project idea.

Thanks to David Karabelnikoff, and PC Peri for their efforts in filming each of the interviews, as well as Caleb Heymann for editing two of the interviews into video pieces. Thanks to Dan Handleman of the Flying Focus Video Collective for providing the vehicle to air the two videos on public access television. Appreciation also goes out to Kay Reid of the Institute for Tribal Governance (ITG) and Charles Hudson of the Columbia River Intertribal Fish Commission (CRITFC) for advice on filming interviews and enabling use of the ITG's camera equipment.

Inexpressible gratitude goes out to my incredible family. Especially my wife, Lisa Lammert, who offered unwavering love, patience, support, and tolerance of the perpetual excuse, “sorry, I have to work on my dissertation.” Lisa, I'm so grateful for all the joy you bring into my life. And to my parents, Ronald and Sherry Hall, thank you for instilling in me a set of values that embrace a broader sense of self, and for the stable foundation that has enabled exploration of who I truly am. Appreciation goes to my brother, Loren Hall, who offers an infectiously provocative view and approach to life. As he always says, I'm glad we're on the same team.

Thanks to all the Shambala Warriors of the global community who recognize the profound injustices humanity is perpetuating on the planet and ourselves. I've been enriched by many perspectives, and inspired by visions of hope. I look forward to continuing this journey in your company.

Finally, to All That Is, which enables the marvelous gift of Life. May we be grateful and wise enough to treat this gift with care and respect. 


\section{TABLE OF CONTENTS}

Page

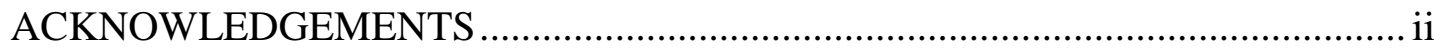

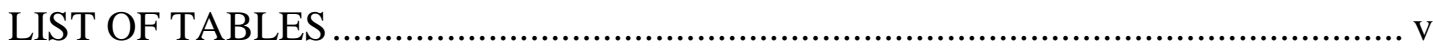

LIST OF FIGURES .......................................................................... vi

PREFACE Author's Perspective ............................................................... vii

\section{CHAPTER}

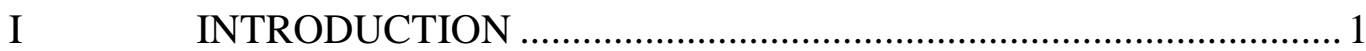

Overview of aims ........................................................................ 3

Rationale for Method .......................................................................... 5

Project Approach .......................................................................... 6

Document Structure ............................................................... 6

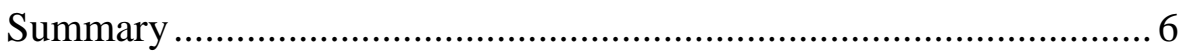

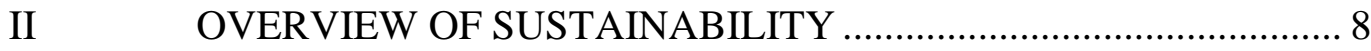

An Unsustainable Trajectory ...................................................... 8

Aligning the Spheres of Ecology, Society and Economy ................. 12

III CULTURE AND WORLDVIEWS .......................................... 17

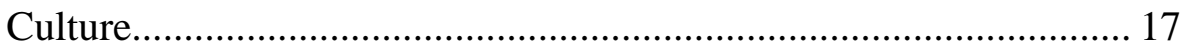

Worldviews ............................................................................ 19

Reconnecting with the problem and purpose of this study .............. 25

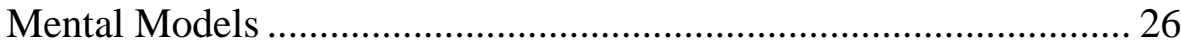

Unique Meaning of Sustainability .............................................. 27

IV ESTABLISHING CONTEXT ................................................. 30

Bioregional focus ..................................................................... 30

Brief History of Native Peoples within Salmon Nation.................... 35

Modern Historical Context .......................................................... 37

Today and towards the future .................................................... 40

V METHODS ...................................................................... 42

Overview of Research Approach ................................................. 42

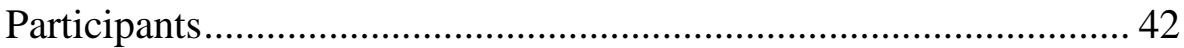

Data Collection: Semi-structured interviews ................................. 44

Data Management \& Content Analysis ........................................ 45

Determination of Quality .......................................................... 50

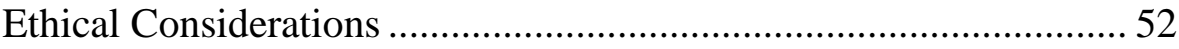




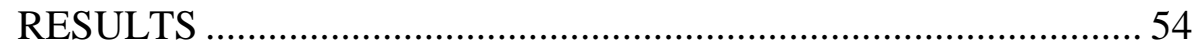

Testimonial Validity Survey Results ............................................ 55

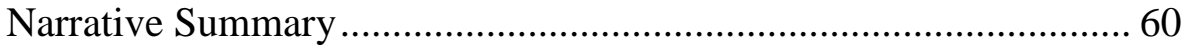

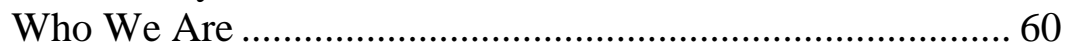

Our Communities.................................................................. 61

"Sustainability:" The word and concept .............................. 62

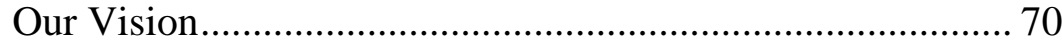

How We Get There .......................................................... 73

Narrative Endnotes....................................................... 78

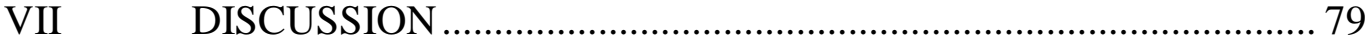

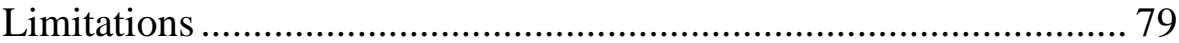

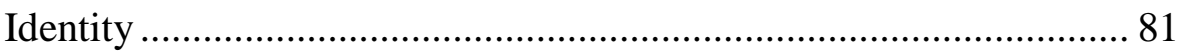

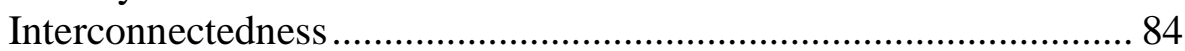

Active stewardship................................................................. 86

Restoring our relationship with the land ...................................... 88

Restoring Community ................................................................. 91

Cooperation: Focus on Bridging TEK and Science ......................... 96

Culture: Enduring Identity ....................................................... 98

Conclusion .................................................................................... 100

Summary of Contribution and Looking Forward ......................... 101

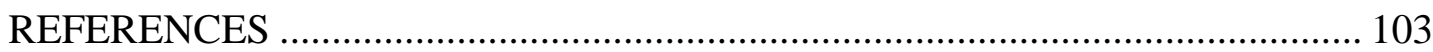

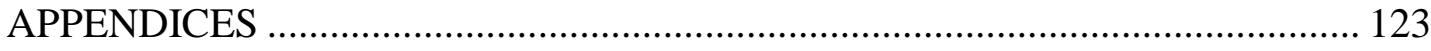

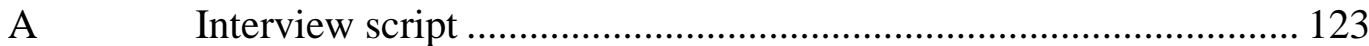

B Disclosure of researcher's relationship with Ecotrust .................... 126

C Initial project summary document ........................................... 128

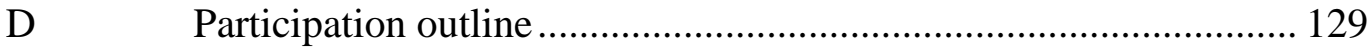

E Letter of informed consent......................................................... 130

F Videotaping letter of informed consent ....................................... 132

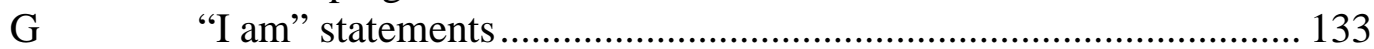

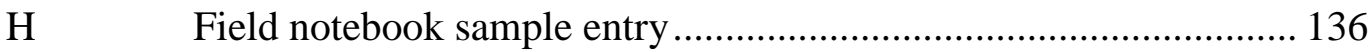

I Testimonial validity survey data .............................................. 137

J Project and narrative critique from Larry Merculieff ..................... 143

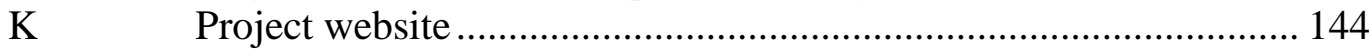

L “Native Perspectives on Sustainability” video pieces .................... 145

M The naw'qinwixw process of the Syilx people ............................... 146 


\section{LIST OF TABLES}

TABLE

Page

1 Comparative terms for Environment, Society and Economy ............ 14

2 Traditional Versus Modern Views................................................. 21

3: $\quad$ Participant names, primary heritages, residences at time of interview, and genders. 44

$4 \quad$ Participant responses to survey question assessing their comfort with their name being associated with the narrative as originally composed.

Response distributions to selected items from the testimonial validity survey.

6

Personal heritages cited by participants .......................................... 60

7

8

Participant use of the term "sustainability"

Excerpted responses to question(s) regarding use of term sustainability and/or "how would you define 'sustainability"”? 


\section{LIST OF FIGURES}

FIGURE

Page

1 Common depiction of the three dimensions of sustainability and their intersection..................................................................... 14

2 Embeddedness of Economy within Society within the Environment............................................................................. 15

3 Embeddedness of Economy within Society within the Environment with Matter, Energy

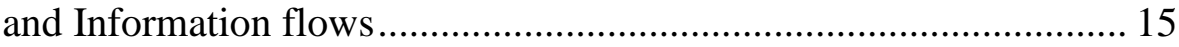

4. The bioregion of Salmon Nation.................................................... 33

$5 \quad$ General movements of goods to and from the Columbia River trade fairs hosted at Celilo Falls................................................ 34

$6 \quad$ Grand Ronde and Siletz Reservations, 1800-1977 .......................... 36

$7 \quad$ Concept Map Symbol Key ............................................................. 50

8 Mapping of topics closely related to the concept of sustainability, and some of their interconnections ............................ 65

9 A view of health as resulting from interaction between healthy environments, healthy foods, healthy people, and healthy communities and cultures ............................................. 75

10 Representation of identity, or sense of self, based upon participant responses with example "I am" statements .................. 82

L1 Screen shot from video "Native Perspectives on Sustainability: Nichole Maher (Tlingit)” ......................................... 145

L2. Screen shot from video, "Native Perspectives on Sustainability: Roberta Conner (Cayuse)”. 145

M1 The balancing of polarities through the naw'qinwixw process of the Syilx people. 


\section{PREFACE}

\section{Author's Perspective}

Despite the best efforts to achieve objectivity in observations, all science, particularly social science, is inherently subjective in practice (e.g., Faulconer \& Williams, 1985; Fine \& Vanderslice, 1992; McGrath \& Johnson, 2003). From the moment we choose a topic based upon personal interests, through the design and observation phases, to the conviction with which we affirm or discount our findings, any given study is, in part, a reflection of the subjectivity of the researcher. Lendaris (1986) refers to each person's "unique set of perceptual filters” (p. 604) as bearing significant influence on both predisposing people to perceive only certain aspects of given data while neglecting other aspects, and how that data is processed and interpreted for meaning. Further, the way we perceive and react to the world then influences the way the world responds to us, thus influencing the type of information we are likely to encounter. Constructivist theorists recognize this phenomenon and the impact it plays on the research process, epistemologically arguing that it is "impossible to separate the inquirer from the inquired into. It is precisely the interaction that creates the data that will emerge from the inquiry" (Guba \& Lincoln, 1989, p. 88). With awareness of the inherent subjectivity that is brought into any research engagement, one strategy is to make aspects of that subjectivity as transparent as possible (Fine \& Vanderslice, 1992). Therefore, I offer some personal background to shed light on the perceptual filters and motivations I

bring to this research. I invite the reader to remain critical in thinking how this project ${ }^{1}$ is a reflection of the researcher as well as that which is being researched.

My ancestry is primarily German and English on my mother's side and German, Irish, Scottish and English on my father's side. I was born in Portland, Oregon in 1978 to parents who moved to the area early in their childhoods. As such, I came to know myself as a native Oregonian. However, through culturally engaged parents and the Portland Public School system, I came to understand that the region I call home has been home to indigenous peoples for many, many generations prior to my family's arrival. These same sources of understanding helped me to develop an appreciation and respect for Native cultures. As I grew older and more educated, my awareness grew of environmental and social injustices that permeate our world. In learning about the displacement of indigenous peoples and the transformation of their lands, I came to see this history as representative of the injustice perpetrated by the expansion of the Western world on other human beings and all aspects of the natural environment.

Given this new perspective on the world, I was left to negotiate my identity. I still identify myself as a native Oregonian and Pacific Northwesterner, but native only in a Euro-centric sense. My ancestry is not Native to this place, and my presence here has come at the expense of others. This realization has generated some internal dissonance as I am unable to escape the thought that my presence is analogous to that of an invasive species. I recognize that my love of this place I call home is shared by many other nonNative people, and that it is unreasonable to expect that we are going anywhere anytime soon. However, our treatment of our recently established home is abusive and

\footnotetext{
${ }^{1}$ Throughout the manuscript I reference this dissertation as a "project" in favor of referencing as "research."
} 
unbecoming. From the thoughtlessness of littering to the audacity of allowing the direct discharge of toxins into local waterways our behavior does not reflect that of an enlightened society. Collectively, I understand such local level acts as contributing to a global pattern of behavior that is on an unsustainable trajectory. This perspective, well supported by research (see Chapter III), is my foundational motivation for engaging in this project.

I am concerned about our present course, but I dream of a day when we have the wisdom to treat our home, wherever that may be, with care and respect to ensure that its vitality is not compromised. I believe much can be learned from the people whose ancestors have inhabited a place for thousands of years. It has been their necessity to live in alignment with the opportunities and constraints that define each bio-geographic place. Today much has changed, and much knowledge and cultural heritage has been tragically lost, including the severance of many relationships with and within the landscape. Many more of those relationships are at risk of being severed. Alternatively, we can proactively work to preserve and restore those relationships. The alternative becomes more probable if we find ways to collaboratively work with and learn from our indigenous friends and neighbors.

In addition to these views, my formal education in the field of organizational psychology included training in the discipline of systems thinking. First, while the dissertation is not a study of organizational life, the reader will find examples from organizational life and citations from organizational scholars whose perspectives afford relevant insight. Systems thinking is a framework for seeing wholes rather than parts, interrelationships rather than separate objects, patterns of change rather than static 'snapshots' (Senge, 1990, p. 68). A systems perspective is independent of and applicable to any field of study. The subject matter of this project demands a multi-disciplinary perspective; this demand is one that I welcome as an opportunity to test my training in systems thinking.

In short, this project reflects my humble commitment to a life-long pursuit of contributing towards the reconciliation of past injustices and to help achieve a promising future for the born and unborn of all species. Towards this end, I maintain some optimism that there remains time to transform the way we think and live towards greater accord with the natural order of this amazing planet we are fortunate to call home. 


\section{CHAPTER I}

\section{INTRODUCTION}

This project was approached with a concern about the trajectory of the human relationship with planet Earth. The Millennium Ecosystem Assessment (MEA) (2005), conducted and agreed upon by 1365 leading scientists from 95 countries, represents a comprehensive account of the state of global ecosystems and the services those ecosystems provide to humanity. The assessment paints a disturbing picture of the present state of and trends in the health of global ecosystems in the face of humaninduced change. The critical message is that if humanity's behavior maintains its present course, the capacity of the planet to provide for human needs will collapse under the weight of human demands (MEA, 2005). Caught in the midst of this predicament are indigenous peoples ${ }^{1}$ across the globe.

Efforts to understand the threats to global ecosystems have revealed the intimate relationships between human socioeconomic systems and the natural environment (MEA, 2005; United Nations Development Program, 1994, 1998). Recognizing the intimacy of the relationship between human systems with the natural environment has drawn attention to the need to create alignment and mutually supportive relationships among ecology, ${ }^{2}$ society, and the economy. The concept of sustainability has been proposed to represent such alignment. While the idea of sustainability is not entirely new, the United Nation's (UN) World Commission for the Environment and Development (WCED) is credited with energizing the concept by defining sustainable development as "development that meets the needs of the present without compromising the ability of future generations to meet their own needs” (WCED, 1987, p. 13). In 1992, the U.N. Conference on Environment and Development (UNCED) in Rio de Janeiro, (i.e. the Earth Summit) called for sustainable development "to ensure socially responsible economic development while protecting the resource base and the environment for the benefit of future generations" (UNCED, 1992, p. 13). As noted by the Union of Concerned Scientists (UCS) in their Warning to Humanity (1992), humanity's ability to address global ecological threats and achieve sustainability will require a major transformation in patterns of behavior at all levels and domains of human activity.

The UCS Warning and call for dramatic behavioral change can be interpreted as a call for new forms of culture. The concept of culture is invoked to capture the holistic nature of human systems, from the more visible and tangible aspects of observable behavior to the invisible and abstract, such as values (Schein, 1992; Triandis, 1972) and

\footnotetext{
1 “Indigenous peoples" is used throughout this paper to refer to those human groups who have the earliest historical connection with a particular geographic region. The terms “indigenous," "Native,” and "Indian” are used synonymously. "American Indian” is used in reference to indigenous peoples of the lower 48 states. The term "First Nation" is used to refer to the indigenous peoples within Canada. "Alaskan Native" is used to refer to the indigenous peoples within the state of Alaska.

2 "Ecology" and "environment" are used throughout this document to refer to the broader context of the natural environment and all relationships and dynamics there within, both human and non-human. This is noted to distinguish use of the terms here with use of the terms "ecology" and "environment" as they frequently appear within the field of developmental psychology in reference to the larger human or social context individual development occurs within (e.g., Bronfrebrenner, 1979). Bronfenbrenner's ideas are directly visited in Chapter VII.
} 
basic assumptions (Schein, 1992), which underlie behavior. Drawing upon the models of culture as articulated by Triandis (1972), Schein, (1992), and Erez and Gati (2004), I present culture as a system of shared meaning that is (a) historically adaptive for the group, (b) perpetuated through learning processes among members, (c) characterized by observable artifacts and behavior, espoused values, and basic assumptions, (d) a multilevel construct from individual to global, and (e) subject to change through top-down and bottom-up dynamics.

Focusing on cultural worldviews, the more abstract and less visible dimensions of culture, differences are noted between the dominant Western culture from some of the generalities that can be made of indigenous cultures (Booth, 2003; James, 2000; Trimble, 1976; Winter, 1996). Fundamental aspects of the Western worldview have been implicated in giving direction to the patterns of behavior that account for the unsustainable trajectory of human society (Cajete, 2000; Capra, 1996; Korten, 2006; Meadows, 1999; Skolimowski,1981; Winter, 1996). Prior to contact with the European world, indigenous peoples throughout North America had formed cultures that were highly adaptive to place. Their needs were met without compromising the capacity of the respective place to continue providing for those needs indefinitely (Cajete, 2000). Their ways of life were formed by, and informed, a way of thinking and value system that stands in contrast with the dominant mentality of modern society, particularly in terms of a stronger environmental ethic (Booth, 2003; Cajete, 2000; Trimble, 1976; Winter, 1996).

Krech (1999) and others (e.g., Ellingson, 2001) challenge, however, the idea that Indian ways of thought and behavior are characterized by a strong environmental ethic. These arguments serve to help differentiate Indians from the contemporary definitions of environmentalists as "nature lovers" and the romanticized visions of living without impact on the landscape. Yet, evidence from Booth (2003) and others (e.g., Cajete, 2000) speaks to the fact that beliefs and practices widely present among Native cultures served to moderate deleterious effects on the landscape (see Chapter 4 for further discussion). Undisputed is that with European contact came a great deal of change: those that survived experienced dwindling options for maintaining traditional ways of life and were subject to assimilation (e.g., Neihardt, 1932). Today, considering the realities documented by the Millennium Ecosystem Assessment (2005), indigenous people also face the risks and uncertainties posed by threats to global and local ecosystems that they are embedded within and rely upon (e.g., Houser et al., 2000).

Recognizing that the Western worldview and behavioral strategies, which have historically served much of Western society as functional and adaptive, have created a set of circumstances that endangers us all, this project sought to contribute to an effort of developing new cultural forms that act in harmony with the larger environmental context. Perhaps the directionality of cultural change can be reversed, and aspects of Native worldviews can supplant the particularly hazardous aspects of the dominant Western worldview.

With the above global concerns in mind, and the broad aim of facilitating cultural change, this project focused on a bioregion in North America defined by the historic Pacific Salmon runs. Within this region people have thrived for over 10,000 years, developing diverse cultural practices united by a common link to salmon (Wolf \& Zukerman, 2003). Prior to European contact the region was home to a large and diverse population, including what is estimated to have been some of the most densely populated 
areas in North America (Kroeber, 1934). This provides testament to the natural abundance of the region. Since contact, the Native peoples of the region have been subjected to patterns of displacement and acculturation, and witnessed deterioration of the region's abundance. However, relative to many other stories of contact between expansionists and indigenous peoples in other parts of the world, many tribes have managed to maintain or reclaim inherent rights of sovereignty, and aspects of their culture have been preserved in the face of assimilation (Wilkinson, 2005). Preserving their rights of sovereignty and culture has been a constant struggle that continues in the present and into the foreseeable future. Survival to date can be largely attributed to the efforts of many great tribal leaders and activists throughout the region (Wilkinson, 2005). It is with such contemporary leaders that this project engaged and explored questions about the meaning of sustainability, their visions of a sustainable future, and how to achieve such a future.

Participants were selected based upon specific criteria; they are individuals who (a) satisfy the definition of indigenous as defined above, (b) have assumed leadership roles at the local community, tribal, regional, national and/or international level, and (c) are highly respected among their peers for the contributions they have made through their work. In fulfilling these criteria, this study primarily included leaders identified through association with the Buffett Award, an annual award ceremony hosted by Ecotrust, a nonprofit conservation organization based in Portland, OR, to honor the contributions of indigenous leaders throughout the bioregion.

The conversations with these participants followed the qualitative methodology of semi-structured interviewing (e.g., Smith, 1995). The interviews sought to tap the mental models, defined as internal representations of reality (Craik, 1943, Johnson-Laird, 2000), of indigenous leaders within the salmon bioregion on topics including: (a) meanings of sustainability, allowing opportunity for alternative terminology to be substituted; (b) concepts of what a sustainable future might look like for a given community; and, (c) actions that must be taken to achieve sustainability, as well as other themes that emerged from the interviews as centrally relevant (see Appendix A for complete interview script). The information obtained through the interviews provides the basis for generating conceptual models of what sustainability means, what it looks like, and how to achieve it from indigenous perspectives.

\section{Overview of aims}

The content from the interviews were organized into four segments coinciding with the interview script: identification of the participants and their community affiliations, the concept of sustainability, visions of a sustainable future, and actions to achieve sustainability. Across the later three segments, a central aim of the project was to identify the patterns of convergence among the interviewees' respective mental models of sustainability, while noting incompatibilities among the perspectives. Towards this end, the software program ATLAS.ti was utilized to facilitate the organization and content analysis of each segment. The details of the methods employed in the collection, analysis and reporting are provided in the Methods chapter. Corresponding to the three segments of the interviews, the products of this project include: 1) a summary of the associated meaning of the term sustainability and any suggested alternative terms, with discussion of divergence among participants; 2) a narrative summary of a vision of a sustainable 
future that was subjected to testimonial validation (Stiles, 1993) by the participants; and 3) a summary of the actions identified for actualizing a sustainable future. Additionally, the researcher worked to develop a few concept maps (Novak 1991; Novak \& Gowin, 1984) to illustrate some primary aspects of the leaders' mental models. As a whole, the project is an attempt to identify and articulate dimensions of a shared mental model among indigenous leaders in the greater Northwest bioregion on the meaning of sustainability, what it looks like, and how to achieve it.

The aims of the project can be seen as serving the functions of contextual and generative research, as defined by Ritchie (2003). The project entails contextual research (also referred to as exploratory or descriptive research, e.g., Marshall \& Rossman, 1999) in that a primary aim was to identify what exists in the minds of indigenous leaders, and to describe and relate their perspectives in a manner that captures the inherent nature of those perspectives (Ritchie, 2003). In each of the segments outlined above, the primary objective was simply to document and represent the information offered by the interviewees in a descriptively accurate manner. Ritchie (2003) cites qualitative methods as particularly well suited for capturing and displaying, with richness and detail, the perspectives of participants in their own terms. A contextual contribution from this study is provided in several forms, including the generation of information useful for (a) mapping the range of elements, dimensions, or positions on the concept of sustainability; (b) describing the meaning that indigenous leaders associate with the concept of sustainability; and (c) identifying and defining typologies of conceptual meaning of sustainability held by indigenous leaders.

Beyond the contextual or descriptive contribution, the project also aimed for a generative or heuristic contribution to social knowledge by aiding in the development of theories and strategies or actions on a contemporary issue (Ritchie, 2003). Within the narrative, and within each individual interview, readers will find generative contributions that include: (a) distinctive conceptualizations and understanding about the fundamental challenge of sustainability and related issues for both Native and non-Native populations; (b) identification of creative solutions and strategies to the problems that pose a threat to sustainability; and (c) identification of actions to improve the effectiveness of existing programs, policies, services, or partnerships.

The project provides a valuable contribution to the regional and international dialogue focused on questions about sustainability. Efforts to understand and work towards objectives of sustainability have increased dramatically in recent years. With effective resource management as a central concern, indigenous peoples have been identified as valuable collaborators for improved management of those resources due to intimate knowledge developed through long-standing relationships with particular places (WCED, 1987). In the greater Northwest bioregion, many Native peoples have maintained or are rebuilding connections with their heritage of living successfully and sustainably within this bioregion. The voices of a number of the individuals at the forefront of this effort are included here. Such inclusion in the sustainability dialogue increases the likelihood of justice for Native communities as well as the development of more enlightened and inspiring policy for the health and sustainability of all society.

Additionally, a particular value of the dissertation lies in its articulation of a positive vision of the future. The value and importance of a vision has been recognized and applied by many, including at the levels of the organization (e.g., Baum, Locke, \& 
Kirkpatrick, 1998), community (e.g., Michaels \& Lopez, 2005) and nation-state (e.g., Howard \& Coombe, 2006). For example, in discussing a process for working with tribes towards self-sufficiency and self-determination, Smith and Anderson (2001) state, "creating the vision of what the future should hold is perhaps the most important step." A well-articulated and inspiring vision bears the potential to give direction to the behavior of individuals and collectives (Ackerman, 1984; Levin, 2000; Senge, 1990). Through the orientation of a vision, human actions take on a new level of purpose and meaning (Ackerman, 1984; Senge, 1990). An important qualifier for a vision to inspire the committed action of many people is that it must be shared; it cannot be a single person's vision imposed upon others (Senge, 1990). This point is of great importance to this project. For even a successful articulation of an optimistic vision for the future, if only held by a handful of prominent leaders, fails if it is not a shared vision. It must still be identified with and embraced by the people who offer their commitment because it reflects not just the desires of the leaders but also the desires of the people. As such, full realization of the potential contribution of this project will require further work in communicating the content among Native and non-Native audiences across the bioregion and exploring receptiveness to it as a shared vision for a sustainable future.

\section{Rationale for Method}

This study used qualitative methods as the primary mode of inquiry and analysis. Qualitative methods were well suited for this project as it sought exploration and discovery of a complex subject-matter rich with dynamic processes and contextually dependent meaning (Morgan, 1997). In addition to the ability of qualitative research to make contextual and generative contributions, such as those identified above, Ritchie (2003) outlines a number of features of phenomena under study that "necessitate" qualitative research (p. 32). This study satisfied several of those features, including: (a) the need for greater understanding of an issue where measurement of extent is not of particular interest; (b) the subject of interest is deeply rooted in the participant's personal knowledge, requiring delicate and responsive questioning to draw the knowledge out; (c) complexity of a subject that is intricate and/or conceptually difficult to relate; and, related to complexity; (d) information was collected from specialists or identified "experts," requiring exploratory and responsive questioning to cover the breadth, depth and idiosyncratic nature of their understanding of the subject. Further, the positivistic assumptions underlying quantitative approaches were deemed incompatible with the project aims and epistemological orientation (see Richardson, 2002; Snape \& Spencer, 2003; and, "Methods"). Along these same lines, a qualitative approach was chosen for this project because, in general, qualitative methods: (1) explicitly acknowledge the subjectivity of the research process; (2) minimize abstraction of information from participants; (3) honor the relativity of truth; and, (4) do not impose authority of the final product (see Lincoln \& Guba, 1985; McGrath \& Johnson, 2003; Merrick, 1999).

Semi-structured interviews. The semi-structured approach was chosen as it enables a researcher to gain a detailed account of participants' perceptions, beliefs or accounts of a subject of interest (Smith, 1995). The method assumes that what respondents offer in their answers "does have some significance and 'reality' for them beyond the bounds of the particular occasion," and "represents a manifestation of their psychological world, and it is this psychological reality that one is interested in” (Smith, 
1995, p. 10). Overall, the advantages of the semi-structured approach include: the facilitation of greater rapport and empathy between interviewer and interviewee; allowance of flexibility to cover new emerging areas of the interview; and, production of richer data (Smith, 1995). These advantages outweigh the disadvantages, which include less control of the researcher over the course of the dialogue, more time in completing the interview, and greater difficulty in analysis compared to information obtained through structured interviews or survey responses (Smith, 1995). The presumed disadvantage of sacrificing control of the interview can alternatively be viewed as a strength. By creating the conditions where the respondent was regarded as an expert on the subject, the conversation was open to moving in directions the interviewer may not have previously considered (Smith, 1995). This said, responsibility remained with the researcher to give thought and consideration to the structure and aims of the interviews.

\section{Project Approach}

This project was approached in the traditions of qualitative activist research and social action research, where research is designed not only to explain, but also to actively promote social change (Fine \& Vanderslice, 1992; Lewin, 1948; Montero, 2001). From this perspective, research holds the potential through the process of inquiry and interaction with participants, and through the research products, to serve as a tool for the facilitation of social change. The project idea was developed in part to align with Ecotrust's mission to create Salmon Nation, a place where people and salmon thrive (see Appendix B for disclosure about my relationship with Ecotrust). It is my hope that all people central and peripheral to this project, as co-enablers or simply as readers, find that some aspect of the project informs them, inspires them, or bolsters their sense of hope and optimism for the future.

\section{Document Structure}

The systems science orientation of the dissertation called for a multidisciplinary approach and an emphasis on context and the interrelationship of phenomena. Subsequently, the opening chapters of this document are primarily designed to provide a broad contextual setting for which the interviews took place. In setting such a stage, topics including environmental science, biogeography, and the legal and socio-political history of Native peoples are covered in greater extent than is customary of psychological studies. What may be lost in terms of depth of theory is compensated by breadth of perspective, hopefully encouraging an enhanced appreciation for the fact that all phenomena are interrelated despite apparent separation in time and space (e.g., Swimme \& Berry, 1992).

\section{Summary}

In summary, this project is spurred by recognition of the problem of the unsustainable trajectory of human behavior and the need for transforming our collective patterns of behavior and associated ways of thinking. The project engaged contemporary indigenous leaders in conversations that explored the meaning of sustainability, painted a picture of a sustainable future, and addressed how to bring such a future into reality. Qualitative methods were employed to collect, analyze and report the information obtained towards contextual and generative contributions. A primary aim was to identify 
elements of shared thinking among the participants, and to represent that thinking through accurate and accessible representations. Intentionally pursued in the tradition of social action research and with a multidisciplinary approach, this project sought to inform and inspire towards the end of positive social change for a sustainable and just future. 


\section{CHAPTER II}

\section{OVERVIEW OF SUSTAINABILITY: PROBLEM RECOGNITION AND TOWARDS REMEDIATION}

In fourteenth-century England, the general village structure consisted of homes arranged closely together, in proximity to a communal pasture used to graze livestock. The communal pasture represents the commons, a resource available to every member of the community to provide for individual need. In these villages, one's livelihood was tied to the ability to rear livestock. Clever individuals realized they could enhance their wealth by putting out more livestock to graze at a limited personal cost. As more individuals adopted this strategy, the ability of the commons to provide the necessary vegetation to support the livestock became overwhelmed. Without the ability to raise livestock to provide for human sustenance, village after village collapsed. What appeared to be a logical practice from the individual perspective proved to conflict with the interests of the community as a whole, and thus eventually conflicted with the interests of the individual. The story provides an exemplary case of the tragedy of the commons (Hardin, 1968), and illustrates the inter-relationships that exist between the three domains of ecology, society, and economy. Ultimately, when talking about human systems, these three domains should be considered as inseparably interconnected.

In this chapter I review the current state of the planet's ecosystems and note some of the critical relationships between those ecosystems and human activity. The idea of sustainability is introduced as the development of patterns of living in balance with the planet such that human needs are satisfied today without jeopardizing the capacity of the planet to meet the needs of future generations (Hawken, 1993; World Commission for the Environment and Development, 1987). Achievement of sustainability will require dramatic transformation in the mode of human behavior (Union of Concerned Scientists, 1992). Several conceptualizations that offer guidance towards achieving sustainability

are touched upon, including the Natural Step framework (Robert, 1997), the triple-bottom line (Elkington, 1998), the representation of overlapping spheres, and an open-systems perspective (Rands, Ribbens, Casagrande \& McIlvaine-Newsad, 2007). The chapter then transitions to a discussion of the concepts of culture and worldviews and their role in giving direction to behavior.

\section{An Unsustainable Trajectory}

At this moment in history there may be a tragedy of the commons occurring on a global scale. Earth's ecosystems that provide services vital to sustain life (e.g., clean air, fresh water, and productive topsoil) are in jeopardy due to dramatic changes instigated by a growing human population (Goudie, 1990), particularly over the last 50 years (Scholes, Hassan, Ash, \& Condition and Trends Working Group, 2005). Human activity threatens fresh water supplies through over-consumption and the introduction of sewage, infectious agents, synthetic chemicals, organic chemicals, mineral substances, sediments, radioactive substances, and heat into waterways (Malmqvist \& Rundle, 2002; Strandberg, 1971; Vorosmarty et al., 2005). Significant atmospheric changes have been observed, attributable to greenhouse gas emissions (e.g., $\mathrm{CO}_{2}$, methane, chlorofluorocarbons (CFCs), nitrous oxide), aerosol generation, deforestation, over-grazing, and other human 
activities (Goudie, 1990). One of the major environmental concerns is the observed trend of global warming or climate change, resulting primarily from the consumption of fossil fuels and subsequent release of greenhouse gases into the atmosphere (e.g., House et al., 2005 Intergovernmental Panel on Climate Change [IPCC], 2001, 2007). The potential implications of global climate change include a range of effects from extreme and unusual weather patterns (IPCC, 2001, 2007) to an increased risk of disease migration through insect populations that thrive in conditions of warmer temperatures (Epstein et al., 1998, Epstein, 1999). Additionally, increases in global temperatures appear to threaten the survival of coral reefs, which act as important carbon sinks, the loss of which could hasten the climate change problem (Hughes et. al., 2003). A steady stream of new studies adds to the evidence of possible links between current phenomena and climate change (e.g., extreme weather events), and outlines new forecasts of possible consequences of climate change. The problem is considered by many to be the greatest ecological threat of modern time (e.g., Isham \& Waage, 2007).

Human activity is also responsible for a dramatic loss of biodiversity (Mace et al., 2005). For example, extinction rates are the highest on record since the Pleistocene Age, a phenomenon that correlates with human expansion and dwindling areas of natural habitat for many species (Goudie, 1990). According to Myers (1979), the rate of species extinction due to human activity was one every four years from 1600-1900, one per year after 1900, and one per day at the time of his research in the 1970s. The rate of decline appears to follow an exponential growth curve, as it is estimated that the planet now may lose as many as 30,000 species per year (Eldredge, 1998). While these estimates may be conservative or overstated, it is widely agreed that biodiversity is declining at alarmingly high rates (World Wildlife Fund, 2004). Further, Travis (2003) draws attention to the coupling of climate change with the reduction of available habitat as having potentially devastating effects on biodiversity as species attempt to adapt to climate changes with reduced availability of habitable area suited for their biological needs.

The concern raised by these documented pressures of human activity and subsequent loss of biodiversity is that they threaten the health of entire ecosystems (tropical and temperate rainforests, wet lands, grasslands, and oceanic ecosystems) that provide essential life support processes (Goudie, 1990, Millennium Ecosystem Assessment, 2005). Beattie and Ehrlich (2001) illustrate human dependence on the processes of nature (e.g., water purification, oxygen production, generation of nutrients) and the complex web of biodiversity required for these processes to function. They call for the preservation of biodiversity in the name of preserving the services of the processes as well as preserving the opportunity to tap newly discovered and yet unknown services nature may be able to provide. Recognizing human dependence on natural systems, Beattie and Ehrlich (2001) state, "Conservation is not just for environmentalists, it is everyone's business." They go on to pose the question: "Who can afford to ignore the natural processes that keep us alive?” (p. 225)

To summarize the current predicament, the Earth's resources are in decline, and human demand upon those resources is increasing. Already our demand exceeds the Earth's carrying capacity, defined as “the maximum (load) of a given species that can be supported indefinitely in a defined habitat without permanently impairing the productivity of that habitat” (Rees, 1996, p. 226). Fundamentally, the trend is unsustainable and will be reversed either through conscious human action to reduce 
demands and restore the natural resource capacity, or through the collapse of the human systems that are dependent on the Earth's commons.

Understanding of the environmental problems we face today and the threats they pose for the future requires an appreciation of the inter-relationships between natural systems and human systems. As noted by WCED, "There are not separate crises: an environment crisis, a development crisis, an energy crisis. They are all one” (1987, p. 20). For example, one of the primary messages of the Millennium Ecosystem Assessment (MEA, 2005) is that ecological changes such as pollution, climate change and variability, invasive species, and changes in land use have meaningful direct effects on the well-being of humans and social systems. The reverse also holds true: problems as experienced by humans in fulfilling basic needs can exacerbate environmental problems (MEA, 2005), and greater human consumption by the materially wealthy further strains environmental integrity. Below I expand on the argument of disproportionate impacts by the materially wealthy and the impoverished, but first, I suggest that such behavior should be viewed in the context of the macroeconomic system.

Considering the global economy, and speaking in terms of context, the natural environment and human society provide the context within which the economic system is embedded (see more below). However, traditional modes of economic thinking and practice fail to account for the costs imposed on the social and environmental dimensions upon which the economy ultimately depends (Hart, 1995). Rather, the objective of growing financial capital compels efforts to reduce labor costs, to exploit natural resources, and to externalize the cost of environmental and social impacts (Hawken, 1993; Korten, 2001; Schnaiberg, 1980). It should be acknowledged that a functioning society depends on a healthy economy to provide the stable conditions necessary to meet human needs, including governmental capacity to provide social welfare and security. Subsequently, individuals and government become servants to the ceaseless expansion demanded by the dominant economic model of the times (Schnaiberg \& Gould, 1994). While short-term societal benefits are attained, there is inherent conflict between the contemporary agenda of economic expansion and the preservation of environmental integrity. Caught within this dynamic are people from all walks of life who, through pursuit of a livelihood and in consuming goods and services, reinforce unsustainable economic practices.

The financial elite disproportionately contribute to environmental degradation through direct financial investment driving economic expansion and extensive personal consumption. One measure of human impact on the environment is the ecological footprint, a tool that measures the amount of biologically productive land required to support the resource demands and absorb the waste products of an individual, city, region, country, or entire human population (Rees \& Wackernagel, 1994; Venetoulis, Chazan \& Gaudet, 2004). The total human ecological footprint is primarily a factor of consumption and population. In the United States, where material wealth is high, the world's largest ecological footprint is observed at 9.57 global hectares (23.6 acres) per person (Venetoulis et al., 2004). If the entire world's population (roughly 6.6 billion) lived at U.S. standards of consumption, it would require 59.4 billion hectares of productive land to support such patterns of consumption. With only 8.8 billion hectares of ecologically productive land, nearly six more planet Earths would be necessary to provide the carrying capacity for the entire global population to live with the 
consumption rates of the average U.S. household. In general, developed countries with greater material wealth have larger footprints than less developed countries (Venetoulis et al., 2004).

While per capita footprints are generally lower in less developed countries, poverty in these regions drive greater growth in population (Meadows, 1986), the other critical variable in the total human ecological footprint. Additionally, as recognized by the United Nations Development Program (UNDP, 1994; UNDP, 1998), and consistent with the MEA account, people in impoverished conditions will seek to satisfy their basic needs via whatever means are available to them. In other words, people in situations of poverty have increased dependence on ecosystem services and materials, which can compromise the health and capacity of those ecosystems, further straining the well-being of the people and creating conditions conducive for interpersonal conflict (MEA, 2005). This translates to impoverished people contributing to rapid deforestation (as witnessed in regions of the world's tropical rainforests), over-fishing, poaching of endangered species, engaging in unsustainable farming practices, manufacturing goods and products without regard for environmental impact, and general disregard for local and international environmental regulations. This pattern provides justification for both the free-market calls for broader expansion and liberalization of economic activity (e.g., Gwartney \& Lawson, 2002) and calls to share wealth more equitably and ensure economic viability for impoverished people (e.g., Doppelt, 2003; MEA, 2005). The latter argument is generally more qualified, stating that such efforts should not necessarily be pursued at the expense of expanding the reach of economic activity into ecologically sensitive or protected areas. Additional political strategies for handling the issues of social and environmental injustices have been proposed, such as proposals from the World Social Forum (see Leite, 2005), but have generally received less political support within developed countries to date.

This phenomenon of poverty placing strain on the environment is not limited to developing countries; the same trends can be seen in the United States. For example, many small towns throughout the Northwest are struggling to survive due to declines in the logging industry, which has served as the cornerstone of these local economies. Availability of high-yield forests has waned after decades of heavy harvesting and as support has galvanized for the preservation of public lands and the protection of endangered species. However, pressures on these forests are increasing as recent economic downturns have fostered an atmosphere of increased political support for tapping into natural resources as a means of economic stimulation (Oregon Natural Resource Council, 2002; Robinson, 2004).

The works and examples cited above represent the development of an understanding that in order to gain a more comprehensive appreciation of the forces that threaten the environment, consideration must be given to socioeconomic factors. While the most glaring impacts occur at the extreme ends of the wealth spectrum, both populations (the rich and the poor) and everyone in-between are merely participants in a larger economic system that fails to directly value social and environmental impacts. Recognizing the intimacy of the relationship between human systems with the natural environment has drawn attention to the need to create alignment between the economic, social, and ecological dimensions. The concept of sustainability has been proposed to represent such alignment and now becomes the focus of discussion. 
Aligning the Spheres of Ecology, Society and Economy

Relatively recently, a movement has arisen to create a symbiotic, mutually supportive relationship among ecology, society and the economy, to provide the conditions necessary for a "sustainable" human society. While the idea of sustainability is not new, the United Nation's (UN) World Commission for the Environment and Development (WCED) is credited with energizing the concept by defining sustainable development as "development that meets the needs of the present without compromising the ability of future generations to meet their own needs” (WCED, 1987, p. 13). In 1992, the U.N. Conference on Environment and Development (UNCED) in Rio de Janeiro (i.e., the Earth Summit) called for sustainable development "to ensure socially responsible economic development while protecting the resource base and the environment for the benefit of future generations" (UNCED, 1992, p. 13). Following the same line of thought, Hawken (1993) characterizes sustainability as an economic state where the demands placed upon the environment by people and commerce can be met without reducing the capacity of the environment to provide for future generations. On an ethical level, "sustainability means leaving the world better than you found it, taking no more than you need, trying not to harm life or the environment, making amends if you do" (Hawken, 1993, p 139). As noted by the Union of Concerned Scientists (UCS) in their Warning to Humanity (1992), the ability for humanity to address these issues will require a major transformation in patterns of behavior at all levels and domains of human activity. The magnitude of the challenge faced by the sustainability movement is well illustrated by the reflections of William D. Ruckelshaus (1989), former director of the Environmental Protection Agency (EPA):

Can we move nations and people in the direction of sustainability? Such a move would be a modification of society comparable in a scale to only two other changes: the Agricultural Revolution of the late Neolithic and the Industrial Revolution of the past two centuries. These revolutions were gradual, spontaneous, and largely unconscious. This one will have to be a fully conscious operation, guided by the best foresight that science can provide. If we actually do it, the undertaking will be absolutely unique in humanity's stay on earth. (p. 167)

The UCS Warning and the words of Ruckelshaus speak to the need for both a new level of understanding and consciousness about our interaction with the world, accompanied by significant changes in behavior. As public and private interests strive to come to an understanding and a more complete conceptualization of sustainability, a variety of different models have been developed to aid the effort to understand and guide behavioral change to ultimately achieve sustainability. A few of these conceptions include the Natural Step Framework (Basile \& Rosenblum, 2000; Robert, 1997; Rosenblum, 2000) the triple-bottom-line (Elkington, 1998), and graphical representations such as the spheres of convergence (see more below). These and other conceptualizations each have their merits and weaknesses (see Upham (2000) for review of the Natural Step Framework, and Brown, Dillard, \& Marshall (2002) for a critique of the triple-bottom line). Reviewing various approaches to understanding sustainability one finds variance in consideration of the difficult questions of sustain "what?", "for 
whom?”, “at what cost?”, and "how?” One perspective takes an anthropocentric stance, which is motivated by an interest in human survival, and the survival of other living systems in so far as human systems are dependent on them (e.g., as reflected in the concept of ecosystem services emphasized by the MEA, 2005). Another perspective on sustainability calls for an ecocentric view which values all life on par with human life (e.g., Capra, 1996). The ecocentric stance neither elevates humans above other forms of life, nor subsumes human interests to some external environment. Rather, it celebrates the unity and interdependences of the community of life. To be sure, degrees of variation exist between the anthropocentric and ecocentric stances.

Of the various conceptions, I will discuss only one at any length-the graphic depiction of three spheres of convergence. It is one of the most widely used tools for communicating the concept of sustainability and, as I will argue, provides a limited and distorted representation of the basic frame of reference needed for understanding and meeting the challenge of sustainability. Further, I argue that the level of change called for by the UCS (1992) and understood by Ruckelshaus (1989) entails fundamental change in culture that includes the worldviews that give direction to human behavior. Thus, this will be followed by coverage of the concept of culture, further followed by identification of differences in world views between Native and Western cultures. Through this exposition, a Native perspective will be identified as potentially valuable in the ongoing effort to meet the challenge of sustainability.

Converging Spheres. As noted earlier, sustainability has been characterized as a movement to create a symbiotic, mutually supportive relationship among the three domains of environment, society, and economy. Here "environment" constitutes ecosystems, natural resources and generally all things non-human. "Society" constitutes human systems and networks of relations among communities of people. "Economy" represents the human system of production, distribution, and consumptions of goods and services. Attendance at most workshops or introductory seminars on the subject of sustainability will likely expose participants to some version of Figure 1, used to illustrate the objective of a mutually supportive relationship among the three domains. As an indicator of the popularity of its usage, a search of "sustainability" through Google's Images option results in images of unmistakable resemblance to Figure 1 at a volume greater than any other particular image. This figure is presented to help orient people's thinking towards the potential for alignment of the three spheres as named above, or other terms used more or less synonymously (see Table 1). As the logic goes, for sustainability to be achieved, there must be equal consideration of and alignment among each of the three domains. Exclusive consideration of one sphere, or coupling of two spheres, is not sufficient. Sustainability requires decision-making and action at the nexus of the three spheres. The figure can be particularly useful as a decision-making tool for testing whether a particular decision is more heavily weighted towards one domain or another. 


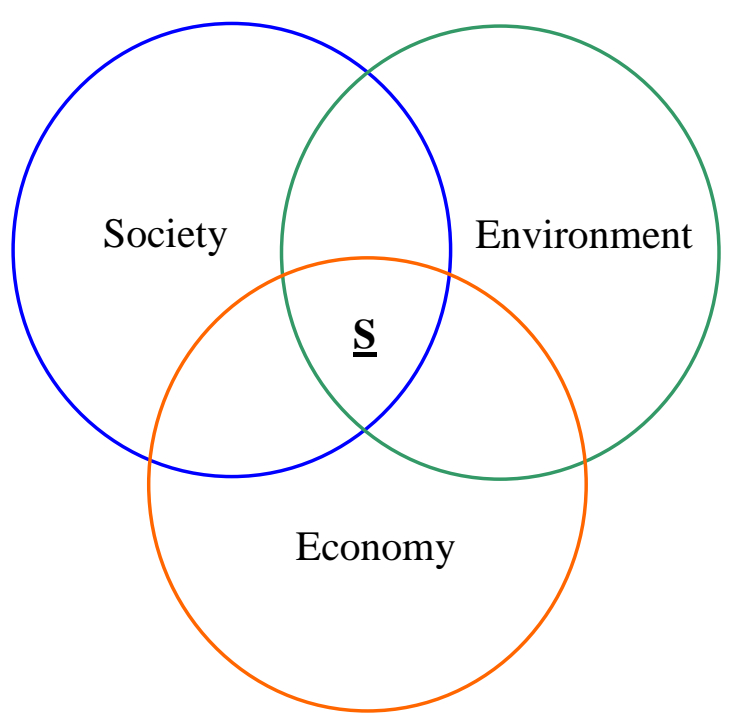

Figure 1. Common depiction of the three dimensions of sustainability and their intersection. Operating within the nexus of the three dimensions is the stated goal of the sustainability (ㅇ) movement.

Table 1

Comparative terms for Environment, Society and Economy

\begin{tabular}{llll}
\hline Primary terms & & \multicolumn{2}{l}{ Comparative terms } \\
\hline \hline Environment & Ecology & Place & Land \\
\hline Society & Equity & People & Labor \\
\hline Economy & Economics & Profit & Capital \\
\hline
\end{tabular}

The three spheres depiction is of value insofar as it serves to bring the social and environmental dimensions into awareness and promote the potential for synergy among the three spheres. However, Figure 1 is misleading because it implies that the spheres exist with a greater degree of independence than is true of reality. Zwick (unpublished) asserts that "a system is 'incomplete' in so far as it has an environment, separate from itself, that is not only relevant to itself but actually obligatory for its existence” (p. 49). Zwick uses the term "incomplete" to capture the notion that the system is dependent on its environment for its very existence. As such, the economy is inherently constrained by the parameters of society and the natural environment. In other words, the economy is fully dependent on the context provided by society and the natural environment; removal of one or the other effectively eliminates the existence of the economy. No system can persist, let alone optimally, without considering the constraints imposed by the environmental context.

Embracing these ideas, Figure 2 illustrates economy's embeddedness within society, and the society within the natural environment. Figure 2 illustrates that the whole of the economy is subsumed by the whole of the society, both of which are contained within the whole of the environment. In other words, there are no aspects of the economy that exist outside or independently of society or the environment. Building upon Figure 2, Figure 3 begins to illustrate a perspective that is fundamental to appreciating the questions raised by the sustainability movement; the open systems view, 
which emphasizes the interconnections between the system and its environment. The open systems view, largely indebted to Bertalanffy (1968) and Miller (1978), asserts that there are matter, energy and information flows between the system and the environment. It implies that the system's ability to preserve itself as an entity, embedded but distinct, depends on preserving the flow of matter, energy and information between the system and environment. From the economic system's perspective, rather than seeing the social and environmental dimensions as some added, confounding pair of constraints, the economy should acknowledge them as constraints that have been present all along and indeed are essential for its own existence. Their presence has simply been marginalized in the awareness of economic decision-makers at all levels (i.e., individually to institutionally). Strengthening the economy without accounting for the inherent constraints of its social and environmental context is a temporary practice at best. This recognition of the misalignment between the economic system and its larger environment is a central tenet the modern sustainability movement.

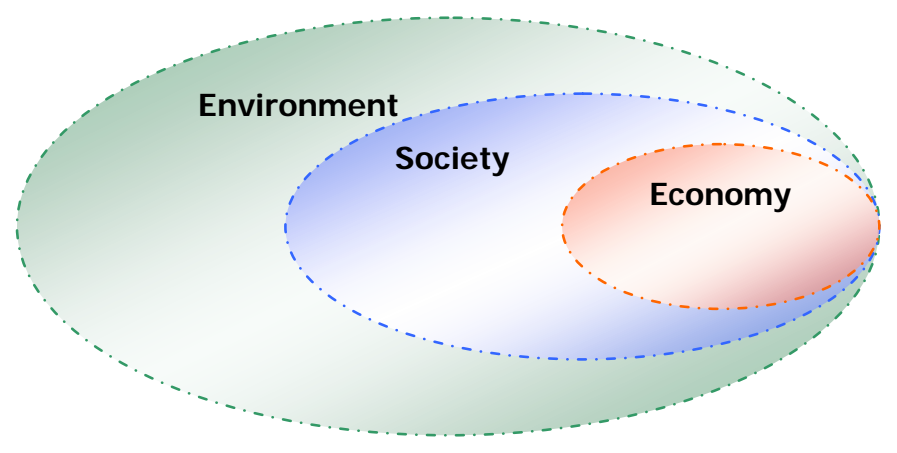

Figure 2. Embeddedness of Economy within Society within the Environment.

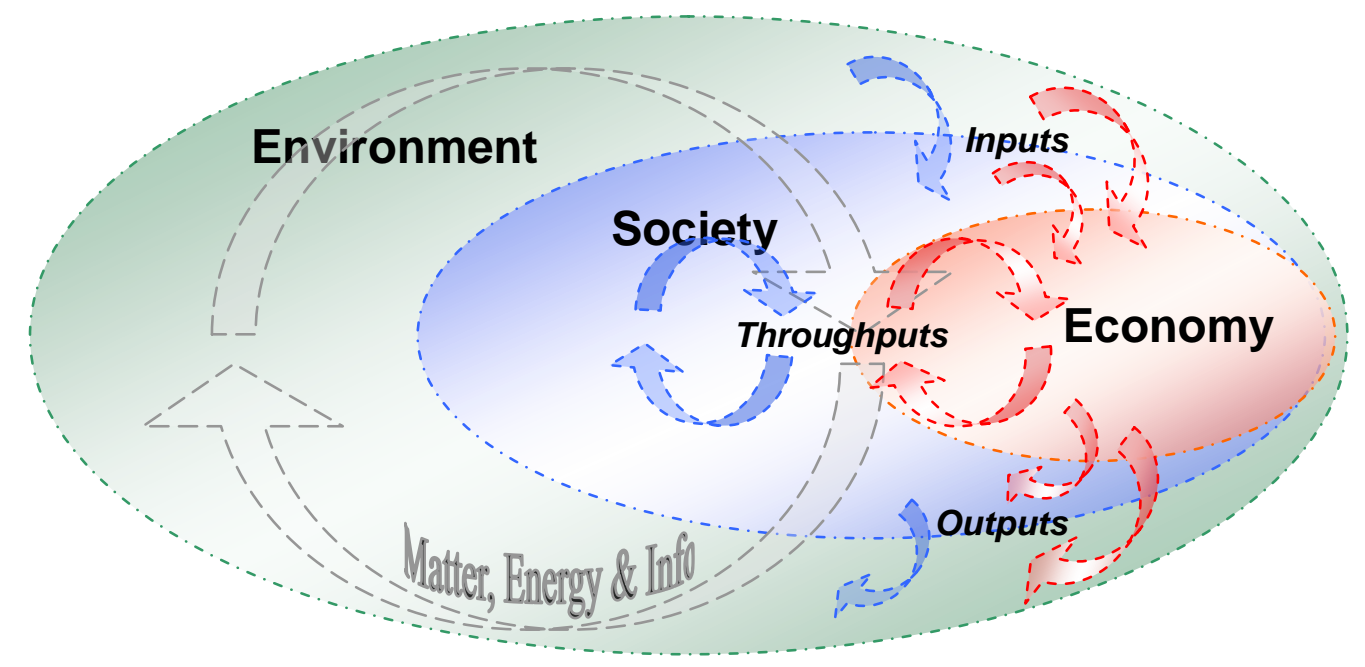

Figure 3. Embeddedness of Economy within Society within the Environment with Matter, Energy and Information flows. Economy as a key mechanism for delivering Matter \& Energy \& Info flows to meet the needs of society. 
The open systems emphasis on environmental dependence makes the theory well suited for utilization with conversations around the concept of sustainability. Indeed, the modern sustainability movement is largely indebted to the ideas promulgated by the open systems movement. For example, the logic underlying the Natural Step Framework (Robert, 1997) is largely based upon an open systems view. The influence and value of the open-systems framework is also evident in one of the more recent conceptualizations for sustainability as offered by Rands, Ribbens, Casagrande and McIlvaine-Newsad (2007). Approaching the question of how to achieve an ecologically sustainable organization, these researchers draw on the open systems framework by identifying key levels and the inputs, throughputs, outputs, feedback, values (as an integrator), and strategies (as a coordinator), that apply to each level. The imprint of the open-systems theory on this study can be observed in the emphasis on relationships in the interview script (see Appendix A), and in the general theme of embedded systems interacting with environmental context.

The problem of an unsustainable trajectory has been outlined here, as well as the need for dramatic change in human patterns of behavior. The economic system has been identified as operating in discord with its larger environmental context, yet little has been said about the psychological roots of the problem. As articulated by scholars and theorists such as Skolimowski (1981), Winter (1996), Capra (1996, 2004), Meadows (1999), Cajeta (2000) and Korten (2002), the threat of ecological collapse has its roots in a cultural worldview. The chapter that follows develops this argument further, as well as explicates the concepts of culture and worldviews, and offers some comparison between Native and Western worldviews. Aspects from Native worldviews are also discussed as potentially valuable in contributing to the psychological foundation of sustainable patterns of thought and behavior. 


\section{CHAPTER III}

\section{CULUTRE AND WORLDVIEWS: THE ROOTS OF THE UNSUSTAINABLE TRAJECTORY}

In reflecting upon the vast social and ecological problems facing modern humanity, an increasing number of scholars and theorists have identified that the roots of these problems lie in the prevailing worldview of the dominant culture (e.g., Cajete, 2000; Capra, 1996, 2004; Korten, 2006; Meadows, 1999; Skolimowski, 1981; Winter, 1996). While the precise choice of terminology varies, the general logic is consistent: the dominant cultural worldview is characterized by a set of basic assumptions and beliefs about the nature of reality that gives direction to individual and group behavior which is destructive to Earth's ecosystems. Therefore, successfully transforming the behavioral patterns and reversing the course of the unsustainable trajectory requires cultural change at the level of worldview. Such statements demand clarity on the constructs being invoked and articulation of the relationships among those constructs. Towards this end, in this chapter I provide a conceptualization of culture, and then take a closer look at worldviews as the core dimension of culture, respectively referred to as: basic assumptions (Schein, 1992), orienting values (Kluckhohn \& Strodtbeck, 1961), values (Triandis, 1972), mindset or paradigm (Meadows, 1999), or cosmology (Skolimowski, 1981). In this discussion comparisons will be drawn between traditional worldviews as observed among many Native communities with those characterizing the dominant Western worldview.

\section{Culture}

The term culture has a history of wide and varied use in the social sciences (e.g., see Schweder \& Levine, 1984). In reviewing a range of definitions, each of which they credit with having validity, Triandis, Kurowski and Gelfand (1994) endorse working with a definition of culture that suits a particular study's purpose. In this project, the concept of culture is invoked to capture the holistic nature of human systems, from the more objective, observable artifacts and behavior to the subjective, invisible and more abstract, such as values (Schein, 1992; Triandis, 1972) and basic assumptions (Schein, 1992). Drawing primarily on the models of culture as articulated by Triandis (1972), Schein, (1992), and Erez and Gati (2004) I present culture as a system of shared meaning that is (a) historically adaptive for the group, (b) perpetuated through learning processes among members, (c) characterized by observable artifacts and behaviors, espoused values, and basic assumptions, (d) a multi-level construct from individual to global, and (e) subject to change through top-down and bottom-up dynamics.

One of the preeminent organizational culture scholars, Edgar Schein, offers a general definition of culture that will serve as the baseline definition for this project. Schein (1992) defines culture as:

A pattern of shared basic assumptions that the group learned as it solved its problems of external adaptation and internal integration, that has worked well enough to be considered valid and, therefore, to be taught to new members as the correct way to perceive, think, and feel in relation to those problems. (p. 12) 
Schein's model identifies three basic levels of culture from the more visible to less visible. First, the external level of culture entails the artifacts or all things one can directly observe of culture. This includes aspects such as the built environment, language, technology, clothing, told stories as well as behavioral patterns. One layer deeper and less visible lie the espoused values held by the members of the culture. This level entails that which is consciously recognized and explicitly stated by the members of the group as what is right or wrong, and what works and what does not. At the deepest and least accessible level are the basic assumptions the members hold about the nature of reality. These basic assumptions constitute the "ultimate source" of the values and actions of other levels (Schein, 1992, p. 17). Schein notes that a basic assumption usually starts at the level of being a value, and it is through the experience of successfully applying that value towards problems that the value moves into the unstated and unchallenged realm of assumptions. With these three levels in mind, culture implies patterning or integration of a system's "elements into a larger paradigm or gestalt that ties together the various elements that lie at a deeper level” (Schein, 1992, p. 10). Schein notes that culture requires time to evolve, and requires some consistency over that time for it to develop a shared quality. He suggests that culture implies some structural stability to the group: "When we say that something is 'cultural,' we imply that it is not only shared but deep and stable” (Schein, 1992, p. 10).

In emphasizing the stable characteristic of culture, the Schein definition fails to shed light on culture as dynamic and changing. Triandis (1972) offers a model of subjective culture that responds to contextual changes through distal and proximal antecedents, namely physical environment and history as distal and social institutions and practices as proximal. The Erez and Gati (2004) model represents levels of culture from individual to global, and recognizes cultural change can occur through top-down or bottom-up dynamics, as well as cross-level forces such as acculturation (cultural change due to interaction with another culture). By articulating the potential for bottom-up change, their model goes beyond the ecological models, such as that of Triandis, which suggest culture changes only in response to contextual forces-or top-down processes. The implication here is that while higher-order levels of culture (e.g., global, societal) certainly influence lower-level cultures (e.g., group, individual), any level of culture is subject to change via the culture of its sub-systems, including individuals.

The dynamic, multi-level perspective of Erez and Gati (2004) is complimented by an understanding of the psychological significance of culture as discussed by Triandis. For Triandis, culture is the human-made part of the human environment and entails objective and subjective elements. Subjective culture speaks to “a cultural group’s characteristic way of perceiving the man-made part of its environment” (Triandis, 1972, p. 4), and includes perceptions of the group's rules, norms, roles, attitudes, beliefs, and values. These elements of subjective culture then predict behavior within that social context (e.g., Triandis, 1980). Essentially, distal antecedents such as the natural environment and historical events give shape and form to social systems and the proximal antecedents of social life, including occupations, religion, language, place of residence, and other social stimuli. Individuals engage in psychological processes to interpret those proximal antecedents which give shape and form to subjective culture, and the actions individuals engage (Triandis, 1972). Similarly, Kitayama (2002) states that individuals 
engage in active psychological processes to coordinate their thoughts and behavior with the cultural systems in which they are embedded. Consequently, that cultural model becomes an integral part of their psychological make-up. The emphasis here is in the effect of perceivable culture on mind. However, perhaps of greater significance to this project is the effect of mind on perceivable culture.

The meaning systems that constitute culture are not just in the heads of individuals, but also embodied in the artifacts, social institutions and collective patterns of behavior (Kitayama, 2002). The point is that these externalized elements are reflective of the internal meanings of the culture (D’Andrade, 2001; Kroeber \& Kluckholn, 1963; Schein, 1992). Thus, closer examination should be given to these deeper, internal dimensions of culture and the idea that our patterns of behavior and various artifacts are reflective of these deeper dimensions, which I will refer to as worldviews.

\section{Worldviews}

In the above discussion of culture, basic assumptions were identified as a deep, core dimension of culture. For different authors, the choice of terminology varies, including basic assumptions (Schein, 1992), orienting values (Kluckhohn \& Stodtbeck, 1961), values (Triandis, 1972), mindset or paradigm (Meadows, 1999), or cosmology (Skolimowski, 1981). This project employs the term worldview and uses it as synonomous with Kuhn's (1970) famous definition of paradigm as, ”an entire constellation of beliefs, values and techniques, and so on, shared by the members of a given community" (p. 175). Similarly, "the shared idea in the minds of society, the great big unstated assumptions - unstated because unnecessary to state; everyone already knows them - constitute that society's paradigm or deepest set of beliefs about how the world works [i.e., worldview]” (Meadows, 1999, p. 17).

With variations in language, Korten, (2006), Capra (1996, 2004), Meadows (1999), Cajete (2000), Winter (1996), Schein (1992), Skolimowski (1981), and Kluckhohn and Stodtbeck (1961) each argue that worldviews form the core of societal structures. All aspects of culture that constitute a given society are outward expressions of those internally held worldviews. Following this logic, the current state of the planet as outlined earlier can be attributed to the effects of our individual and collective patterns of behavior as guided by our fundamental worldviews. The change that is necessary to turn the tide against the trends of degradation will require a dramatic change in culture at the level of worldview. Speaking of systems behavior, Meadows recognizes that one of the highest points of leverage for change, albeit not necessarily the easiest, comes at this level of changing the worldview or paradigm that perpetuates that system. What is it about the worldview of modern society that can account for the unsustainable trajectory outlined above? Exploring the contents of some aspects of the dominant Western worldview offers insight on the question.

Meadows (1999) cites some of the widely held and seldom challenged assumptions of the dominant paradigm of today, including: (1) growth is good; (2) land can be "owned" by people; (3) nature exists as an expanse of resources to be applied for human purposes; and, (4) homo sapiens are at the apex of the evolutionary ladder. Winter (1996) outlines similar assumptions as held by the Western paradigm on the subject of nature. These assumptions include: (1) nature is composed of inert, physical elements; (2) nature can and should be controlled; (3) individual human beings seek 
private economic gain; and (4) we must "progress." The important point to consider in reviewing these assumptions is that they serve as the cognitive baseline for consideration of what entails an appropriate or inappropriate course of action.

Kluckholm and Strodtbeck (1961) provide a similar argument in identifying orienting values ${ }^{3}$ as principles "which give order and direction to the ever-flowing stream of human acts and thoughts as these relate to the solution of 'common human' problems" (p. 4). Various cultures differ in their position and rank ordering of the five major value orientations, which are as follows:

1) Human nature: Is human nature innately evil, neutral, good, or a mixture? Is human nature changeable or unchangeable?

2) Human-nature: What is the relation between humans and nature? Is it characterized by subjugation to nature, harmony with nature, or mastery over nature?

3) Time: Is greater value placed on the past, present or future?

4) Activity: What is the valued modality of human activities; being, being-inbecoming, or doing?

5) Relational: What is the appropriate modality of human-human relationships; lineal, collateral, or individualistic (i.e., hierarachical, collectivistic, or individualistic)?

Kluckholm and Strodtbeck argue that a culture's stance on each value provides a clear directive for behavior. For example, dominant Western culture views the humannature orientation as mastery over nature. Subsequently, behavior that imposes human's will on nature becomes the valued and accepted course of action, rather than harmoniously working with the forces of nature. For example, the free flowing rivers get dammed for power and irrigation, and air conditioners are installed to change climate rather than reliance on the body's natural mechanisms for maintaining internal homeostasis in the face of external variance. Collectively, the Western value orientations, similar to the set of assumptions as identified by Meadows and Winter, establish justification for actions that allow for extraction, manipulation, consumption, and degradation of the natural environment. In an exercise comparing the dominant Western worldview with those of indigenous cultures, significant differences are salient.

Before proceeding, it is important to acknowledge the fact that individuals and specific sub-groups will often reveal differences in their worldviews from larger group categorizations such as Western or Native. Among North American indigenous peoples, such differences reflect diversity among the various cultures across the continent and varying degrees of cross-cultural contact (see Trimble and Thurman, 2002). Still it holds that the worldviews characterizing European cultures differ in meaningful ways from Native cultures, and that among Native cultures there are patterns of similarity (Booth, 2003; Cajete, 2000; James, 2000; Little Bear, 2000; Trimble, 1976; Winter, 1996). A comparison of these worldview differences is summarized in Table 2. As aptly stated by Little Bear, "Any individual within a culture is going to have his or her own personal interpretation of the collective cultural code; however, the individual's worldview has its roots in the culture” (2000, p.77). In contemporary times, the worldviews of Native

\footnotetext{
${ }^{3}$ The blurriness between values and assumptions is salient in Kluckholm and Strodtbeck's approach. Rather than distinguishing, they speak of variance in the degree to which each value orientation is conscious in the minds of individuals; from the completely implicit to the completely explicit
} 
individuals are likely rooted in both Native and Western cultural forms due to the history of colonization (discussed further below).

In the discussion of traditional worldviews that follows, emphasis is on the subject of the human relationship with nature. This generalized worldview regarding nature among traditional or non-industrialized cultures is acknowledged as an idealized version with likely exceptions to every rule.

Table 2

Traditional Versus Modern Views (adapted from Winter, 1996, p. 55)

\begin{tabular}{lll}
\hline \multicolumn{1}{c}{ View of: } & \multicolumn{1}{c}{ Traditional View } & \multicolumn{1}{c}{ Modern View } \\
\hline \hline Nature & Alive; imbued with spirit & Mechanical; made up of bits (atoms) \\
\hline Land & Common & Privately owned \\
\hline Humans & Group member & Individual \\
\hline Human nature & Cooperative & Selfish, competitive \\
\hline Time & Circular & Linear \\
\hline Purpose of life & Harmony; sustainability & Progress; growth; material wealth \\
\hline
\end{tabular}

Traditional worldviews. The work of numerous Native American scholars confirms a set of values and beliefs that stands in contrast to dominant Western values (e.g., Booth, 2003; Cajete, 2000; James, 2000; Trimble, 1976; Winter, 1996). Some of the distinct values of Native cultures identified by these authors include: valuing group cohesion and individual humility; equal valuations of humans and non-humans; circular view of time and events; valuing harmony with nature; belief in a spiritual reality linked with the physical world; valuing tradition above change; and a cautioned view of humans that sees their actions and creations as resulting in both good and harm. Two other central tenets of Native American worldviews include an understanding that all things are related and that the natural world is alive and imbued with spirit (Booth, 2003; Cajete, 2000; Winter, 1996).

Knowledge that all things are related established the greatest challenge for indigenous communities, which was to come to an understanding of the patterns of those relationships and the human place there within. The establishment of such an understanding was the primary objective of Traditional Ecological Knowledge (TEK), or Native Science (Cajete, 2000). TEK is formally defined as "a cumulative body of knowledge, practice and belief evolving by adaptive processes and handed down through generations by cultural transmission, about the relationship of living beings (including humans) with one another and with their environment” (Berkes, Colding \& Folke, 2000, p. 1252). TEK is specific to place and represents the accumulated information about the given place necessary for the survival of individuals and entire cultures. It is experientially formed, meaning that the information acquired has been obtained through direct interaction with and observation of that place. From a Western perspective of understanding, the development and application of TEK can largely be credited for the success of Native peoples in the formation of their systems of understanding and ways of life that by today's standards are widely regarded as sustainable. TEK has recently been discovered by modern scientists as a valuable tool in helping to understand and effectively manage complex ecoystems (e.g., Berkes, Colding \& Folke, 2000).

Part of the perceived reality in giving context to as well as the development and application of TEK is the view that all of nature and the earth as a whole are seen as alive 
(Winter, 1996, Booth, 2003). Allen (1979) goes so far as to say that the fundamental difference between Western and American Indian thinking is that an Indian is one who "assumes the earth is alive in the same sense that he is alive" (p. 233). Citing Merchant (1983), Winter discusses how attributing nature as living established a functional constraint on human behavior in that harm would not be enacted on nature as it would constitute unethical behavior the same way Western society views harming other human beings as unethical.

Beyond ethics, such acts of harming nature beyond one's needs and without proper prayers or rituals were believed to disrupt the balance and threaten the very sustainability of the community. For example:

A Navajo does not say a prayer to the inner form of a deer explaining his need for the deer and asking for the deer's indulgence simply because it is a kind and gracious thing to do; he does so also because it reminds him of the deer's right to life and the necessity for him not to be excessive or overindulgent in his use of the deer, for such behavior could throw the whole world out of harmony and balance and that would be dangerous to his own survival. (Witherspoon, 1977, p. 180)

The constellation of traditional values and beliefs related to ecology provides the basis for the idea and practice of ecological reciprocity (Booth, 2003; Cajete, 2000; LaDuke, 1993). Ecological reciprocity entails the ethic of complimenting any act of taking from the world with symbolic and material offerings in return. Imagining such an ethic in practice on a large scale, one can easily appreciate it as providing a strong foundation for ensuring balance in the functioning of human relationships with ecological systems. Cajete acknowledges that Native cultures applied technologies that transformed the landscape in their efforts to survive. What distinguishes the Native employment of technology from non-Native employment is that the former "aspired to live in accordance with an ideal of reciprocity with the landscape, guided by cultural values, ethics, and spiritual practice” (Cajete, 2000, p. 183).

The claims that American Indian cultures are characterized by a strong environmental ethic have not gone unchallenged. Krech (1999) wrote a provocative piece, The Ecological Indian, which deliberately challenged the contemporary view of Indian as living in harmony with nature. Krech, through review of anthropological evidence, sites American Indian behavioral practices that conflict with contemporary definitions of "ecological" and "conservation" as established in the $20^{\text {th }}$ century by the environmental movement. For example, Krech argues that Indians significantly contributed to the Pleistocene extinctions of about 11,000 years ago, while acknowledging the greater impact of climate change. Krech also discusses evidence of extensive use of fire for hunting, communication, and aggression, "without regard to ecological consequences" (p. 120). Krech further illustrates a series of practices that can contemporarily be viewed as wasteful, and exploitative, but make sense when viewed through a cultural relativistic lens. For example, the belief that beavers would continuously remain available if their bones were treated respectfully allowed the potential for unregulated trapping of beavers with possible threat of extermination.

Krech's book is controversial (see Harkin \& Lewis, 2007), has generated claims of being a product of motivated anti-Indian sentiment (Deloria, 2000), and arguably 
displaces attention from the cultural devastation of European invasion and the accompanying ecological degradation. However, Krech successfully makes a case that a view of North American Indians as passive inhabitants of an unmanipulated "wilderness" is a mythical idealization. The success of Krech's argument largely lies in the definition of the term "ecological," employing modern Western vernacular of a "truly ecological Indian... as one who did not change, damage, or irrevocably alter his ecosystem” (as stated by Booth, 2003). Such an argument is difficult to contest, as Native Americans clearly impacted the landscape through the process of making a life, as does any species. His portrayal of Indians as active agents, participating in an ongoing, dynamic relationship with the landscape is legitimate and valuable to our understanding. However, many believe that Krech's arguments go too far in claims that the Indian practices were on course for inevitable environmental deterioration, which the addition of a European presence merely helped hasten (e.g., Deloria, 2000; Pennybacker, 2000). A wide body of literature that illustrates settlers' accounts of a vast "Eden” with great abundance of forest cover and wildlife suggest that Indians were either light in their footprint or were sophisticated landscapers (Pennybacker, 2000), or some combination of the two.

This ongoing debate operates with a distinction between the concepts of sustainability and conservation. Sustainability entails long-term and enduring use of resources without collapse of those resources, while conservation demands intentionality in conservative use of resources, or sustainability by design (Smith \& Wishnie, 2000). Reviews by Smith and Wishnie (2000) and Hames (2007) conclude that examples of sustainability are widespread, but it appears uncommon that Native peoples intentionally practiced conservation. They argue that with some exceptions (e.g., Hunn et al., 2003), sustainability was likely a result of low population density, technological limitations, and minimal external consumer demand. As discussed by Campbell and Butler (in press), the criteria for demonstrating conservation are stringent and present significant measurement challenges. They argue that the conclusion that conservation was rare may be due to a reality that conservation was rare, or due to flaws in the measurement strategies. Additionally, researchers tend to be quick to conclude that resource sustainability does not imply intentional conservation, but collapse of a resource base indicts the absence of conservation, even though other forces beyond human decision-making impact resources (Campbell \& Butler, in press). Further, Hames’ conclusion is based predominantly upon evidence from the post-contact era, which could have been responsible for the breakdown of those systems that facilitated conservation, a possibility Hames cites himself. The conclusions drawn by Hames (2007) and Smith and Wishnie (2000) appear to place little weight in the emic data such as oral histories, which may speak to but lack physical demonstration of conservation by design. Whether indigenous peoples of the region lived sustainably due to circumstance or by design is a difficult question to answer empirically, leaving it open to continued debate.

Reflecting on the overall debate, it is at least equally inaccurate to claim that the vast majority of Native peoples lacked a worldview that valued and respected the living landscape as it is to romanticize the pre-European Indian as exemplars of modern conservationist thinking. Both perspectives are insensitive to the historical and cultural contexts of the Native way of life prior to European contact. Also, Booth (2003) identifies a problem with the debate itself, as "to talk about the 'ecological' or 'non- 
ecological’ Indian is to talk of stereotype” (p. 341). Two non-Native concepts are imposed - that of Indianness, and that of ecological or "good" interactions between humans and their environments. First, stereotyped expectations of an Indian are not real and deny Native peoples the position of peoples with real lives situated with real problems, opportunities, and choices to be made (Booth, 2003). Second, the Western definition of ecological goodness is based on the nature-lover's appreciation of the landscape, removed from daily interaction. Language such as "nature" and "wilderness" convey the idea of remote separation and distinction from human life. While we are never truly removed from the land, Western language and cultural assumptions do not serve to create a conscious experience of our inherent connection. Conversely, the indigenous ways of life did and do entail intimately living within and "making a living" off of the land. It is precisely in such active engagement entailing utilization and alterations of the environment that creates the conflict of applying the Western ideology of "environmentalism” to Native practices. Such practices of utilizing resources for subsistence and economic gain certainly runs counter to the modern environmentalist agenda of establishing conservation areas free from human influence (see Booth, 2003 for examples of conflict between the environmentalist agenda and Native interests).

I share Booth's sentiment that non-Natives still have much to learn about living with the landscape, and that valuable lessons can be learned through consideration of Native psychological worldviews and examples of behavioral practices. From my perspective, this is the question of real relevance to today: whether aspects of Native worldviews foster a sustainability agenda and inform effective responses to the ecological and social challenges of the modern age? This question renders questions about conservation and ecological practices of yesterday secondary, while retaining relevance of historical and modern worldviews among Native peoples that can potentially promote conservation practices today. Two examples include the idea of ecological reciprocity noted above, and the aspiration of aligning human ways with the natural order (e.g., Cajete, 2000). Similarly, Booth (2003) provides a series of examples from Cree efforts to conserve resources to stories of the Trickster that warned against the foolishness of overconsumptive practices. Woven into these examples, at the crux of Booth's entire thesis, and where I see significant value for the cultivation of a sustainable future, is an explicit understanding of human embeddedness in and dependence on the natural world. Such understanding reflects a sense of self held among many Native cultures.

Sense of Self. In the Western view, the self is an independent agent operating in a material world governed by mechanical processes. The self (and humans in general) is separate from nature. The resources of the land and spiritless plant and animal forms are available to be controlled and exploited for human purposes. In contrast, the Native view of self is fundamentally characterized by the inability to separate the individual from the larger environment in which that individual is embedded (Booth, 1996, 2003). As captured in the phrase "we are the land" (Allen, 1979, p. 191), the indigenous view of self is defined by the unique characteristics of place where the people have formed intimate patterns of relationships. The self is seen as not independent from, yet not the same as nature. Self is viewed in the context of an intimate understanding of the reality that survival depends upon the natural world, of which humans are an inseparable part (Booth, 1996, 2003). 
The Native view of self reflects an understanding that humans are participants in the biogeological synthesis (Croizat,1964), where reciprocal dynamics in space and over time between organisms and the environment give shape to the organisms, and also those organisms in concert with one another help shape the landscape (Welsh, 1994). As such, each place takes on a uniqueness that is the product of this dynamic interaction, and each organism embodies the uniqueness of that place. Cajete (2000) speaks to how this interaction results in both the physiological and psychological development of people who live in place for extended periods. As a result, indigenous peoples of various locales not only shaped those locales, but were also shaped by them and came to reflect the characteristics of that place in their physical form, cultural practices, and inner-psychic world. It is this depth of connection and identification with the land that can help us to understand why "the displacement of Native Americans from their lands, and the subsequent damage to that land, was, and is, so socially and psychically devastating” (Booth, 1996, p. 3).

\section{Reconnecting with the problem and purpose of this study}

The extended discussion of worldviews is intended to complement and supplement the preceding portion of the conversation on culture, and further shed light on the roots of the current ecological crises facing humanity. A great challenge of unprecedented cultural change is required to mitigate the ecological crises (e.g., Ruckelshaus, 1989). As shared, and deeply integrated in behavioral practices and artifacts, culture may only be susceptible to change in response to extended exposure to new environmental and/or internal conditions of new meaning to produce new sets of assumptions, values and behavioral norms (Schein, 1992). Consider that the dominant global culture is characterized by a pattern of basic assumptions that "has worked well enough to be considered valid [italics added]” (Schein, 1992, p. 2). Triandis (1994) notes that in the face of contextual changes dimensions of culture that were adaptive in the past may not be adaptive at present or in the future. At present, humanity is experiencing contextual changes, such as, global warming, increased toxicity in the food and water supply, decreased productivity of the ocean's fisheries, etc. Attention to the scientific monitoring of these changes provides informational feedback that our assumptions and behaviors are in fact invalid strategies that are not working well enough for long-term adaptation and survival.

However dysfunctional, culture and associated worldviews serve to provide cognitive stability in the context of a dynamic and uncertain world (Schein, 1992). The discrediting of an assumption is likely to result in anxiety and defensive responses. Successfully managing such responses is facilitated if new views and understandings are made immediately available to supplant those being discredited. The worldviews held by many indigenous cultures may offer alternative assumptions and ways of viewing self in relation to the world that better serves our long-term interests. Berkes (1999) captures the point well in stating:

The challenge is to cultivate a kind of ecology that rejects the materialist tradition and questions the Newtonian, machinelike view of ecosystems...The indigenous knowledge systems of diverse groups...provide an alternative view of ecosystems. This is a view of an ecosystem pulsating with life and spirit, 
incorporating people who belong to that land and who have a relationship of peaceful coexistence with other beings. (p. 182)

With this challenge in mind, the basic aim of this dissertation is to draw out and represent the mental models, internal representations of reality (Craik, 1943), of contemporary indigenous leaders regarding the subject of sustainability. The concept of mental models is introduced from cognitive psychology as closely related to the idea of worldview. The gestalt of one's mental models entails one's worldview. The hope is that indigenous community leaders who are actively engaged in cultural and ecological restoration may offer valuable insight and perspective on the current context of environmental concerns and the interplay, for better or worse, with cultural worldviews. By articulating the mental models of these contemporary indigenous leaders, this project seeks to stimulate dialogue that encourages reflection on mental models and worldviews in relation to issues of sustainability.

Before proceeding, it is important to acknowledge that contemporary Native peoples have had varying degrees of influence from both Western and traditional Native cultures. It is not assumed that the contemporary Native leaders who participated in this study simply provide worldviews that are exemplary of pre-European contact. Rather, it is understood that in the modern context the participants have been influenced by both to varying degrees. This fact actually gives greater merit to the potential value of their mental models to address modern issues as they have perspective on both the Western culture and their respective traditional cultures. Such dual awareness may enable greater insight into the particularly relevant aspects of our mental models that we should be most attendant to. As this chapter has sought to establish, avoidance of a widespread collapse of the global commons will require a shift in the dominant Western culture and the underlying worldviews. Paraphrasing the famous Einstein quote, problems cannot be solved at the same level of consciousness at which they were created.

\section{Mental Models}

In the need for a shift in the dominant culture and worldviews, the term "mental models' has been invoked and only briefly defined. The phrase now deserves closer examination and explication. "Mental model" is a phrase that is attributed to the Scottish psychologist Kenneth Craik (1943), who proposed it in reference to psychological representations of reality. Drawing from Craik and Piaget (1952, 1983) I define mental models as internal representations of reality that are formed through experience yet remain subject to change, are drawn upon to interpret novel situations and inform decision-making and action, and vary in the extent to which individuals are consciously aware of their form and influence on thought and behavior. These representations reflect an individual's assumptions, images, and stories held with regard to aspects of life including people, objects, events or anything else. These models, small-scale representations of reality, serve in helping to interpret and anticipate events.

Mental models of the world and self are formed through any and all personal experiences, including early life learning in the home, exposure to media and other dimensions of culture, interaction with the natural world, as well as through personal imagination (Johnson-Laird, 2000). Their formation subsequently acts as a filter moderating our perceptions and our subsequent responses with the world. "What is most 
important to grasp is that mental models are active - they shape how we act... Why are mental models so powerful in affecting what we do? In part, because they affect what we see" (Senge, 1990, p. 175). Expose two people with different mental models to the same set of sensory stimuli and they will selectively attend to different aspects of the stimuli and form different perceptual interpretations that inform behavioral actions. For example, if a developer and a nature enthusiast were to each look at an unspoiled natural landscape, the first would likely view it in terms of its potential for development, while the latter individual would likely see it in terms of its inherent value and worth as a natural landscape. Clearly, these two different perceptions would predict different courses of action if either of the individuals were given authority over the fate of the landscape.

This idea of mental models draws close comparisons to Jean Piaget's notion of schemas (Piaget, 1952, 1983). Schemas are the "pliable mental molds into which we pour our experience” (Myers, 2001). As we continuously seek to further understand the world, all new information is interpreted from the frame of reference and understanding established by our existing schemas. Piaget employed the term assimilation to capture the process by which we interpret new information through existing schemas, or our current understanding of the world, regardless of whether the schemas accurately reflected reality. However, if a new experience is inconsistent with a given schema we may modify the schema to better represent the aspects of that experience, a process Piaget termed accommodation. These concepts of assimilation and accommodation are invoked here for the purpose of making the point that people raised with orientation towards the Western worldview have the capacity to accommodate their mental models when new information and experiences are encountered, such as those documented by the Millenium Ecosystem Assessment (2005).

Generally, much of humanity's internal representations of reality are in need of serious accommodation, particularly in terms of our view of ourselves in relation to the natural world. In aspiring to give representation to the mental models of Native leaders on the subject, perhaps this project can contribute towards such needed accommodation. Before offering more details on the methodology to be employed, it is important to discuss a key aspect of my mental model on the subject, namely, that sustainability can be understood as a challenge towards self-preservation.

\section{Unique Meaning of Sustainability}

My studies of the topics of sustainability, open-systems theory, and cultural worldviews (particularly in terms of sense of self) have lead to the development of a mental model that understands sustainability as fundamentally about a challenge towards self-preservation. Subsequently, the meaning of sustainability takes on unique form for any given entity that considers the subject. In other words, the answer to the question, "what does sustainability mean for us?" is going to entail a unique answer reflecting the distinct characteristics of the entity posing the question and how it views it-self and its essential relationships with its environment. The view of self informs decisions that must be made about what to sustain, for whose benefit, at whose cost, and how.

Few would argue with the statement that people act in a self-interested manner. Where variability is observed is in terms of the scope of which self is viewed as isolated and separate, or as united through relations with the broader world. Consider: Many 
organizations engage sustainability initiatives because they have made the connection between their ability to maintain operations with the preservation of the external resources and services provided by the community and natural environment (e.g., Nattrass \& Altomare, 2002). At the far end of the spectrum are people who embrace the deep ecology movement and see their individual self within the self of the larger whole, defined through historic and ongoing relationships and commonalities (Booth, 1996). Cultivation of a broader sense of self through building awareness of those relationships and commonalities with all things naturally inclines actions towards preserving the integrity of the planet as a whole. Similarly, the focus of tribal efforts in the Northwest (NW) towards preserving the salmon runs can be at least partially understood in these terms; as an effort to preserve the tribal identity, which is intimately woven with the salmon (e.g., Novak, 1998). In each example, pursuing sustainability is a matter of enlightened self-interest, and the particularities of action vary in accordance with the view of self.

Discussing sustainability as self-preservation and entailing unique meanings is not to say that there are not principles of sustainability that can be identified and applied across a broad swath of humanity. Rather, it suggests that the degree of relevance of each of those principles, and the answers regarding how to apply them to each subject's unique identity and context will certainly vary. For example, consider two businesses in two different industries; one in manufacturing, the other in hospitality. The types of raw inputs, work processes, on-the-job risks to human safety and health, potential wastestreams, and other aspects of their business operations are significantly different. Thus, each organization's environmental and social impacts are going to be significantly different. Change efforts will need to be appropriately tailored to address each organization's unique relationship with their environment while preserving viability as a business operation. Plus, the organizational culture of each will be different and require strategies of communication and implementation that are sensitive to culture. In short, there is no one-size-fits-all or cookie-cutter answer to what sustainability means to any given entity.

Despite unique meaning to each entity, that meaning can be derived from shared sources, such as some of the influential conceptualizations of sustainability mentioned above (e.g., the triple bottom-line). Also, each entity might find commonalities with other entities in terms of their identities and the subsequent meaning of sustainability. Consider the organization in the hospitality industry invoked by the example above. This organization will share much in common with other organizations in the hospitality industry, and thus, there is likely to be a greater degree of convergence in the meaning of sustainability among these intra-industry organizations. They have arisen out of the same market-based system, operate according to the same economic rules, provide essentially the same services, rely on many of the same resource inputs, engage in many of the same internal processes, and generate many of the same waste-streams. If they were able to set competitiveness aside, a difficult proposition under the incentives of the current economic system, these organizations could learn tremendously from one another in striving cooperatively towards achieving sustainability.

Each indigenous community of the greater NW faces significant challenges and an uncertain future. The specific answers and solutions to these questions must be derived from their own internal identity. However, aspects of this identity may also be 
shared through commonalities in worldviews, biogeography and history. The point being, there is potential value for exploring the thinking about the meaning of sustainability across the region of the NW of North America. A shared perspective can lead to more integrated and coordinated agendas for tribes across the region, translating to greater leverage and influence in major policy issues, and making local decisions in accord with larger-scale interests in mind. These potentials may be on the level of specific policies or it might be on the level of general principles and common philosophies. Exploring what those potentials might be is an aspect of the aims of this project.

As an example of such potential, consider the Columbia River Intertribal Fish Commission (CRITFC) and the Northwest Indian Fisheries Commission (NWIFC), which are organizations serving tribes with treaty fishing rights in the Columbia River basin and Western Washington, respectively. Each organization was based on common links in identity among member tribes, including: Native heritage and preserved fishing rights, geographic proximity, existence in the same legal contexts, and perhaps most importantly, dependence on salmon. Their work seeks to preserve fishing rights, preserve salmon, and ultimately preserve tribal ways of life and the tribal identities those ways reflect. By uniting their efforts through sharing of resources and expertise, and speaking with one voice, both commissions have been relatively effective in their missions against huge odds. A larger region that spans across greater political boundaries and geographic terrain may not have the level of cohesion enjoyed by CRITFC and NWIFC, but as noted in the preceding paragraph, the need and value exists for finding commonalities and increasing the effectiveness of the Native voice in addressing issues concerning sustainability.

This project's conversations with contemporary indigenous leaders were engaged with an eye towards building shared understanding and identifying commonalities in meaning. In the chapter that follows, I explore some of the elements of shared history and biogeography across the region to set the stage for these conversations. 


\section{CHAPTER IV}

\section{ESTABLISHING CONTEXT: BIOBEOGRAPHY AND HISTORY}

To have an informed conversation about the future, we must pause and reflect upon the geographical and historical context in which this conversation occurs. "Without context, words and actions have no meaning at all” (Bateson, 1979, p. 15). This project engaged a focus on the bio-region defined by the range of where the Pacific Salmon run. It is within this geographic context that the indigenous leaders were sampled, and commonalities sought from their perspectives about sustainability. This coverage of geographic and historical context is relevant to this project as the identity of many indigenous people is intimately woven with the land and their heritage. The individuals participating in this project live in this region, and many have either witnessed first hand or directly been involved in the recent history covered below. To fully appreciate and understand the perspective they provide through the interviews, we must view it in relation to this context.

In a brief coverage of context of such a large geographic region and historical time-scale, generalities are inevitable and should not be taken to suggest homogeneity of place and peoples. With the truth of diversity in mind and acknowledged when essential, this section also seeks to establish a sense of commonality and interconnectedness of places and stories across the region and over time. First, I will discuss the idea of a bioregional classifications and the logic of using such a focus, then, I will discuss some of the characteristics of the bioregion relevant to this project. I will follow this with a brief overview of the history of Natives in this region and the present day circumstances, with an eye towards the future. Effort is made to distinguish some of the major differences between the lower 48 states, Alaska and British Columbia, particularly in terms of historical context. First, we consider the biogeography that unites this vast area.

\section{Bioregional focus}

The perspective of biogeography is well captured by the metaphor offered by Croizat (1964), "space, time, form: the biological synthesis." Welsh (1994) credits Croizat's metaphor and work for providing an integrated view of the unified dynamic among these three domains, where "organic life is a constantly changing process expressed simultaneously across geographic space and on the time line of history" (p. 99). The idea of employing a bioregional focus to understanding place and living organisms there-within has an academic history that dates back at least to the $19^{\text {th }}$ century (e.g., Schouw, 1823 \& Swainson, 1835; as cited by Welsh, 1994), and has maintained a rich legacy in the discipline of biogeography through contemporary times (see Welsh, 1994). The logic of this approach is based upon the idea that reciprocal dynamics in space and over time between organisms and the environment give shape to the organisms, and also those organisms in concert with one another help shape the landscape. As such, each place takes on a uniqueness that is the product of this dynamic interaction. "Dry and wet seasons, daily light and climatic cycles, and the complex interrelationships of living organisms all contribute to the uniqueness of a given place and determine what species of plants and animals exist there” (Welsh, 1994, p. 98). Understanding the 
uniqueness of place and the interrelationships within becomes important for engaging in effective management strategies of aspects of that place deemed valuable to human populations. "A bioregional focus is required to manage natural resources knowledgeable and wisely while preserving the integrity of natural systems” (Welsh, 1994, p. 99).

If employing of a bioregional focus is so critical to effective management, then one might ask why such a strategy is not more widely employed today. A few examples illustrate that the idea has received attention, and perhaps may be gaining footing in modern times. One example comes from the late $19^{\text {th }}$ century, and entails a proposal presented to the House Select Committee on Irrigation. John Wesley Powell (1891) recommended the creation of sub-governmental units to be formed in accordance with "hydrographic basins" and, "then let the people of each such irrigation district organize as a body and control the waters on the declared irrigable lands in any manner which they may devise” (p. 256).

Powell's recommendations were not implemented in the states, but the idea survived and moved from idea to reality in New Zealand where a bioregional governance strategy has been implemented using watersheds as the defining boundaries for their regional governments or councils. With some oversight from the central government, each of the 12 councils is empowered with making decisions on water allocation and land use decisions that effect water quality (Hobbs, 2004a). New Zealand recognized the centrality of effective water management to issues of social justice, economic development, and environmental protection (Hobbs, 2004b). Through the watershed organization of governance, decision-making determining the quality of that watershed was placed in the hands of unified bodies, eliminating much of the potential conflicts that can arise when disparate governmental agencies are vying for utilization of the same resource.

Another example of thinking in terms of biogeography is seen in the work of the World Wildlife Fund, which has developed a mapping of the Earth's terrestrial biodiversity into regions (Olson et al., 2001). Olson and company (2001) coin these biogeographic areas as ecoregions that encompass relatively large geographic areas each with unique compilations of species and communities of life as they roughly existed prior to human modification. This example is included to illustrate two points: first, how regions have been identified based upon empirical evidence of biological diversity across geography; and, second, to highlight how such regional classifications are subject to fluctuating boundaries, yet are relatively stable and provide useful management applications (Welsh 1994).

Sensitivity to the fluctuations and understanding of the management potential of the biogeographic approach requires attention to both temporal and spatial scales. At a very fine temporal scale (shorter periods of time), we can witness patterns of behavior, and annual cycles. When time is considered in terms of roughly decades (considered relatively fine scale), this ecological time scale enables spatial study of existing plant and animal species, their dynamics (e.g., population fluctuations), and specifics of contemporary features of the local terrain. At coarser scales, observations are made of evolutionary or geologic change, where study is oriented on aspects such as geological history and fossil records (Welsh, 1994). This project will operate at an ecological time scale by guiding participants in an exercise of envisioning sustainability taking form in 
the foreseeable future, where fluctuations in populations of various species are plausible, as well as fluctuations in behavioral practices and aspects of entire cultures.

Matters of scale also apply to the spatial dimension which range from very focused areas of biogeography (e.g., a specific marsh), to areas defined by similar vegetation patterns across an area (e.g., Redwood forests), to much larger regions defined by seasonal cycles (e.g., a temperate climate), purely geographic characteristics (e.g., the Rocky Mountains), or the range of given species (e.g., Pacific Salmon runs). The project presented here encompasses a relatively large biogeographic region defined by the Pacific Salmon runs of North America.

It is important to acknowledge that bioregional classifications are usually anthropogenic constructs that should be judged in terms of the utility they provide towards managing the region (Welsh, 1994). I would add that such utility enabled by the classification serves in accord with the value systems of stakeholders. This project's chosen bioregion does not escape this characteristic of being an anthropocentric construct. The bioregion selected for this project was adopted from the non-profit organization, Ecotrust (see Appendix B for disclosure of my relationship with Ecotrust). Initially organized to protect the temperate rainforests of North America, Ecotrust has expanded its area of focus to the bioregion defined by the historic range of the five species of Pacific Salmon, a place they call Salmon Nation (see Figure 4). The organization's mission is to catalyze the movement for alignment of human activity with the inherent opportunities and constraints of the landscape, such that the region becomes “a place where people and wild salmon thrive” (Wolf \& Zuckerman, 2003, p. 4). 


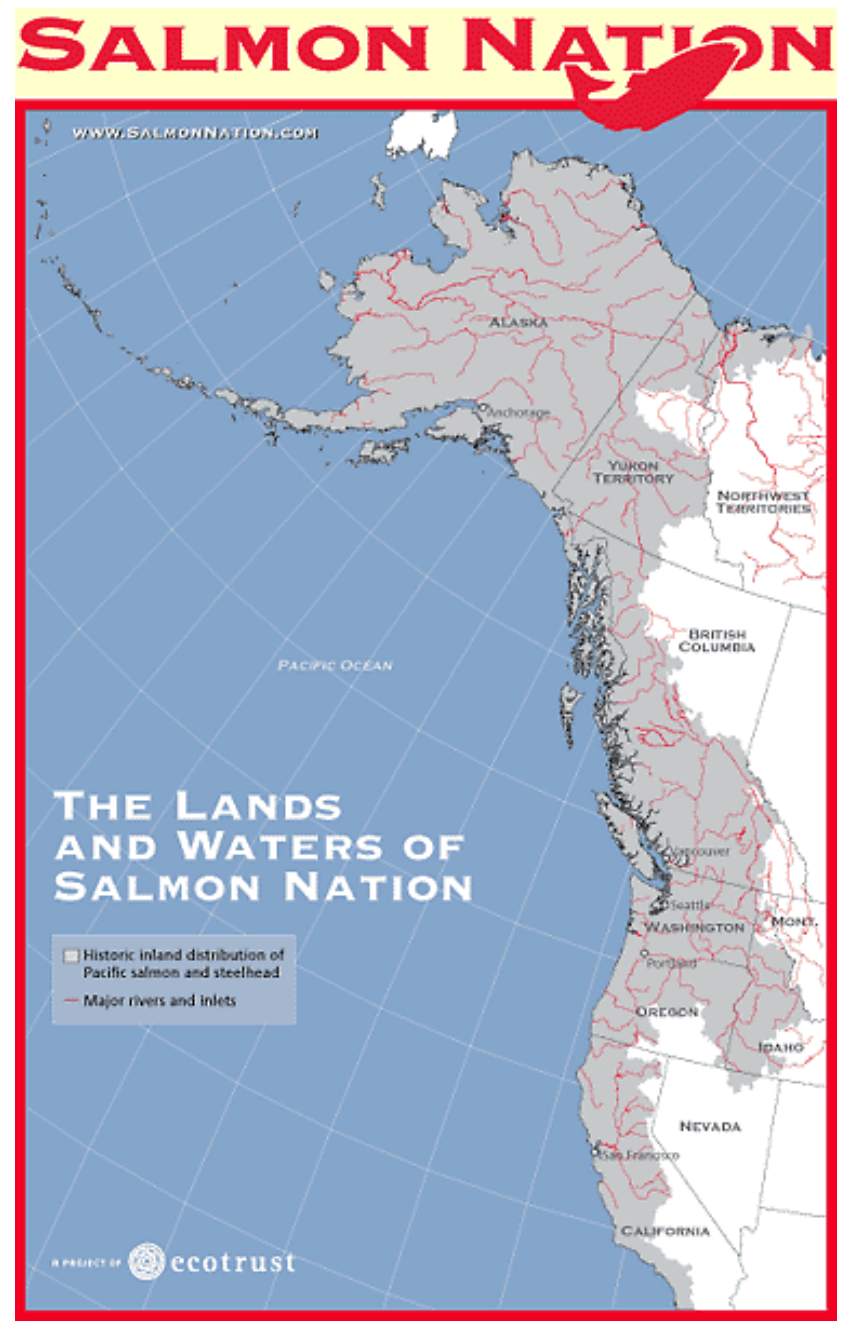

Figure 4. The bioregion of Salmon Nation (from www.sectionz.info/Issue_4/mapping.html)

While the bioregion is an anthropogenic construct, it is based upon some objective realities of geography \& biology; it is in essence a cumulative of watersheds, and it encompasses the historical range of the five species of Pacific Salmon. Defining the region in such a manner is useful and meaningful in three fundamental ways. First, thinking in terms of geographic watershed may enable more cohesive thinking and decision-making on resource issues compared to the fragmented decision-making that can result from arbitrarily defined political boundaries. This idea is supported by the employment and early success of the watershed based governance system in New Zealand.

Second, Pacific Salmon have been identified as both keystone and indicator species. Keystone species are species that are enriching to the functioning of whole ecosystems in a manner that is disproportionate to population numbers (Gende et al., 2002). Their effect is such that loss of the species' presence precedes ecosystem changes, usually including a broader loss of diversity. One hundred thirty-seven species have been identified as directly depending on Pacific Salmon (Cedarholm et al., 2000). The notion of salmon as an indicator species speaks to them as particularly sensitive to 
environmental conditions, thus providing early signs of possible concerns about ecosystem health, such as water pollution (Hilderbrand et al., 2004). Salmon as a keystone and indicator species suggests that they are both of indispensable value to the overall health of the region, and close monitoring of their populations can provide us with important feedback about the general state of ecosystem health.

Third, the value and importance of salmon to peoples throughout the region historically and at present cannot be overstated. Historically, salmon were a cornerstone of the regional economy, serving as a primary source of food and trade. Such importance is illustrated by Figure 5, which depicts the convergence of native peoples onto Celilo Falls, one of the most important fisheries of the $4^{\text {th }}$ largest waterway in North America (Woody, 1999), for annual trade fairs hosted by the Warm Springs and Wasco Indians (Buan C. M., \& Lewis R., 1991). Respecting the centrality of salmon in satisfying basic needs, the salmon were revered in the spiritual and ceremonial lives of many indigenous peoples. That history is alive today as salmon remain central to the physical and spiritual wellbeing of many indigenous people throughout the region.

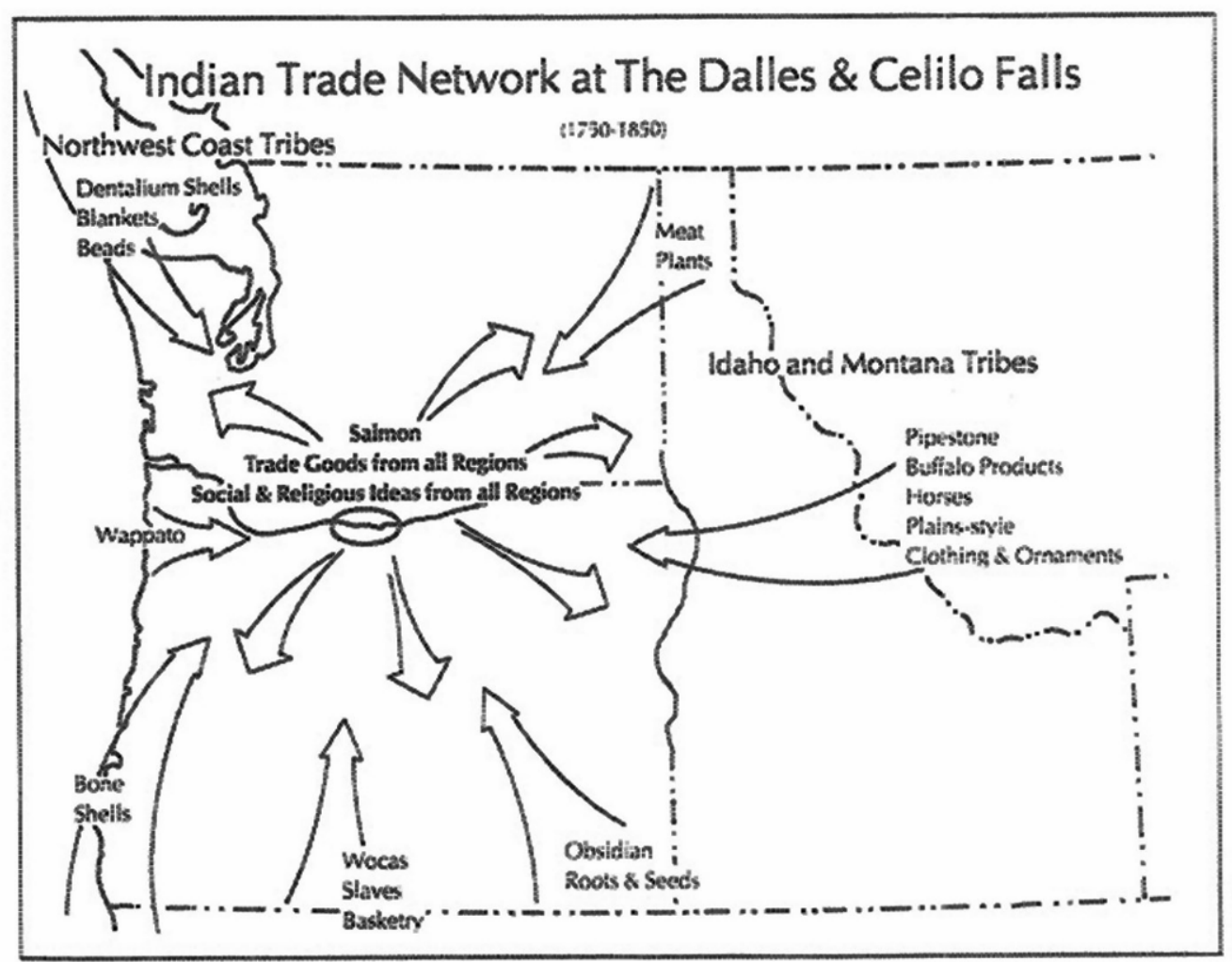

Figure 5. General movements of goods to and from the Columbia River trade fairs hosted at Celilo Falls (from Zucker, Hummel \& Hogfoss, 1983).

Beyond the salmon connection, it is arguable that the region shares other common and interrelated interests, such as energy, water, and commerce. A regional strategy to deal with many of the challenges facing humanity today might be a necessary approach. Such regional thinking is being employed in the area of the Pacific Islands (which encompasses an area greater than the continent Europe plus the Middle East) to address challenges including sustainable development, security and fisheries management (Peters, 
2006). Some questions still remain about the unifying capacity of the idea of Salmon Nation and the pragmatic utility of a bioregion defined by the range of the Pacific Salmon runs of North America. Indeed, one of the applied purposes of this project is to yield information about whether Salmon Nation is a useful construct to inform thinking and strategies within the region. If indigenous leaders throughout the region demonstrate similarity in thought about the future, then that will speak to shared interests across the region. Further efforts would be required to reach out to non-native portions of the regional population to explore whether the similarities extend beyond just the Native leaders. As this project focuses exclusively on perspectives of indigenous community leaders of the region, I now turn to discuss the history of the region as it pertains to Native peoples.

\section{Brief History of Native Peoples within Salmon Nation}

The pages that follow provide a very brief overview of the historical context of the indigenous peoples that have lived within this project's biogeographic region of focus. Emphasis is placed on the word "brief," and the qualifier is added that any finite discussion of history is certain to be an incomplete account. The story of each tribe has its own complexities and idiosyncrasies, but general themes can be observed of migration, adaptation, development of a patterned way of life with the formation of unique culture, contact with European expansionists, encroachment, varying degrees of conflict, marginalization, cultural and political oppression, assimilation, near extinction of tradition, and most recently a path of revitalization in the era of self-determination. In the following thumbnail sketch, the greatest emphasis will be on the modern history of the United States. In this coverage I will largely, but not exclusively, draw upon Charles Wilkinson's book, Blood Struggle: The rise of modern Indian nations (2005), as it provides an authoritative source for American Indian and Alaskan Native history leading into the $21^{\text {st }}$ century. The modern historical context is particularly relevant for this project because the leaders to be interviewed have largely lived this history through their life's work.

Prior to European contact, the region boasted one of the densest population of hunter-gatherer peoples documented anywhere in the world (Kroeber, 1934). Subsistence lifestyles were achieved through a wide variety of means; the choices of plant and animal life depending on the specifics of any given location. Like other parts of the country, with European contact came widespread disease as the indigenous populations had no naturally accumulated immunity to the foreign-hosted ailments such as small pox, measles, influenza and other diseases that previously had been exclusive to the landconnected continents of Europe, Africa and Asia (Boyd, 1999; Cook, 1998). The result was a dramatic decline in population among most tribal groups, estimated to be up to 90\% in the hardest hit areas (Cook, 1998), such as the Lower Columbia and Willamette Valley (Boyd, 1999). The decline in the regional population of Native peoples is primarily attributed to the spread of infectious disease (Boyd, 1999; Cook, 1998; Wilkinson, 2005).

Interpersonal violence also played a role in the decline of indigenous populations, but while battles and wars were waged in many parts of the country, the NW was able to resolve most disputes without large-scale conflict-with some exceptions such as the conflict over the Nisqually treaty and the Nez Pearce War. Rather, facing inevitable 
defeat to the military superiority of the U.S. government, tribes with sufficient internal organization were essentially forced into negotiations. Often tribes were not granted favorable terms of agreement, particularly on the subject of land-base. However, in many cases, especially in the NW, tribal leadership had the foresight to include language in their treaties to ensure "usual and accustomed" hunting and fishing grounds. Despite the establishment of treaties, many of which occurred in the 1850s, the years that followed demonstrated that the word of the treaty did not always govern policy on the ground, particularly if gold was discovered within that ground. In many cases treaties were "renegotiated" without consent from most tribal leaders. A dramatic illustration of how terms established through treaty often eroded over time can be seen in Figure 6, which illustrates the decline of the Grand Ronde and Siletz Reservations from their original range to the point of restoration in 1997. The illustration includes effects of the U.S. policy of allotment, which is explained below. It is worthy to note, that while the treaties of the 1850's were eroded, particularly by the measure of land-base, their legacy is enduring to this day. They provide the basis for legal recognition of tribal sovereignty, federal trust responsibilities, and the rights to accessing lands off-reservation for practices of hunting, fishing and gathering (e.g, US v Winans, 1908). The preservation of these elements has proved essential to the preservation of the tribes themselves.

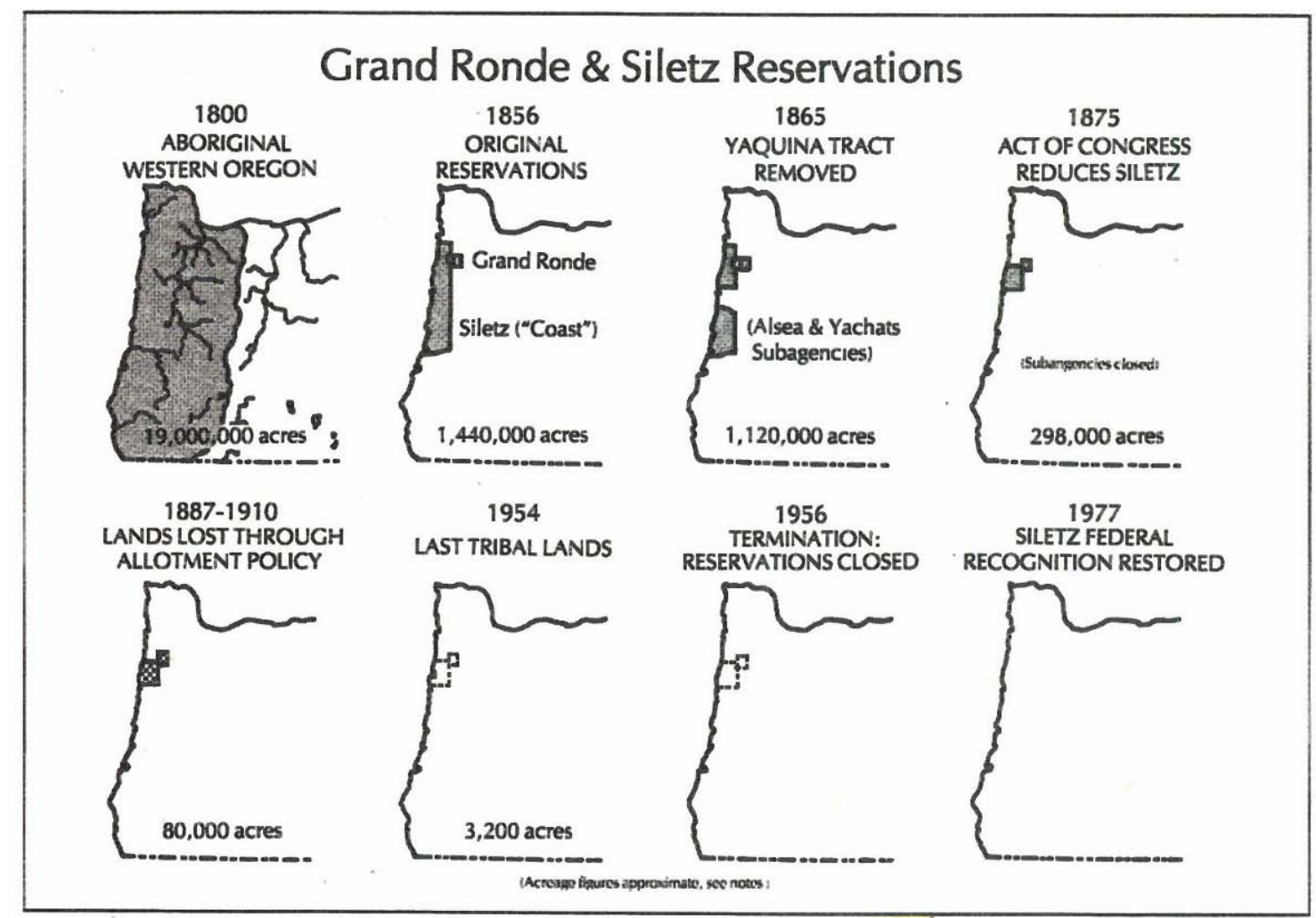

Figure 6. Grand Ronde and Siletz Reservations, 1800-1977 (from Zucker, Hummel \& Hogfoss, 1983). Figure does not illustrate the reallocation of lands as granted by the Restoration Acts of 1977 and 1983 for the Siltetz and Grand Ronde respectively.

In addition to the spread of epidemics and the disregard of treaty obligations, Wilkinson (2005) recounts the deleterious impacts of alcohol, missionaries' conversion 
efforts and suppression of traditional beliefs and practices, assimilation through education, forced transition to the cash economy, and the policy of allotment on tribal ways of life. The General Allotment Act of 1887 was either a well-meaning yet misguided attempt at facilitating tribal realization of the Jeffersonian ideal, or a deliberate attempt to fragment tribal ownership of the land to make way for the voracious appetite of further westward expansion. Regardless, the net effect was a nation-wide decrease in land-base from 140 million acres in 1887 to 52 million acres in 1934 when allotment policies were abandoned. As I discuss below, the policy of "termination" that was to follow proved to pose a fatal risk to even these remaining lands. Collectively, Wilkinson refers to this era from the point of European contact through to the mid 1900's as "the deadening years.”

\section{Modern Historical Context}

In the United States, the experience of Indian people approached a low point by 1953 when the U.S. government enacted a new policy of "termination," articulated in House Concurrent Resolution, 108 (Wilkinson, 2005). The resolution called for the termination of hundreds of treaty agreements with tribes across the country, effectively severing the obligations of providing federal services, and dividing reservation land among remaining members. The move was designed to relieve the federal budget, open valuable reservation land to the promise of development, and purportedly to allow Indian people the opportunity to live in the way of the white man-insisting "Indians to get up on their own two feet, to walk on their own" (Wilkinson, 2005, p. 86). The policy proved to be devastating for Indian peoples, but it also marked a turning point. Faced with the total loss of identity and their traditional ways of life, indigenous leaders across the country began to organize against the policy of termination, and began a movement towards re-establishing tribal sovereignty.

The movement grew out of the most unlikely of conditions, amidst some of the more impoverished communities in the country. Championed by many from across the country, the work took tireless effort and, as Wilkinson (2005) outlines, was guided by key philosophical teachings that held the movement together with values and inspiration. Among the inspiration included the work of Charles Eastman, Black Elk and D'Arcy McNickle. Eastman's The Soul of the Indian painted a portrait of Indian values and worldview, capturing much of the essence of what it meant to be Indian. The words of Black Elk, as presented by John Neihardt in Black Elk Speaks, provided a sense of roots and place in the universe. "To (young Indians) the book has become a North American bible for all tribes. They look to it for spiritual guidance, for sociological identity, for political insight, and for affirmation of the continuing substance of Indian tribal life” (Deloria, Jr., as quoted by Wilkinson, 2005, p. 97). D’Arcy McNickle, whose many contributions included, The Surrounded, They Came Here First, and Wind from an Enemy Sky, enthnographically documented tribal ways, was a vocal advocate for tribal sovereignty, and raised awareness of the communication gap that contributed to the difficulties between whites and Indians. These voices in particular, paid tribute to the Indian way, tribal sovereignty in their homeland, and helped keep alive these ideas during the most difficult of times (Wilkinson, 2005).

Many difficult times were encountered, particularly during the post WWII era characterized by both termination and rapid economic expansion that targeted tribal lands 
for large scale public and private enterprises. Tribal governments were ineffective during this period and learned many difficult lessons about protecting their rights and interests when dealing with the outside society. Previous establishment of the National Congress of American Indians in 1944 proved to be an important step in building the political voice of Indian peoples. The Indian cause was bolstered by the return of a large cohort of WWII veterans who had proudly served and were discontent to be relegated to secondclass citizenship status. Additionally, a new generation of young educated leaders, including Vine Deloria Jr., Clyde Warrior, Henry Adams and Mel Thom, came onto the scene giving Indians intellectual footing and articulation of a purpose for their efforts (e.g., the 1961 Declaration of Indian Purpose).

While the termination era caused significant losses across Indian country, some victories were won and more importantly many lessons were learned. Signs of a burgeoning Indian movement were on the scene, and continued to grow over the course of the 60s. The War on Poverty, accompanied by a key piece of legislation, the Economic Opportunity Act (1964), enabled tribes the right to apply for and utilize federal money without oversight from the BIA or any other entity. Not only symbolic in overcoming the paternalistic relationship with the federal government, this Act enabled the establishment of key infrastructure for tribal organizing capacity (e.g., staff, offices, phones, fax machine).

The 1960's also bore witness to the Civil Rights Movement (CRM). Leaders of the Indian cause were reluctant to join because in some fundamental ways they were not seeking the same objectives. Where CRM aimed for integration, a primary goal of the Indian movement was resistance to forced assimilation. However, the movements shared the desire for basic rights and social justice. The CRM cultivated a national climate of supportive sentiment for minority interests in general, forcing the dominant white society to recognize the humanity of all people. During this time Native leaders also learned through observation and direct participation general organizing skills and activism tactics. It also was an era that cultivated a sense of possibility for change. Perhaps nowhere is such a sense of possibility illustrated as well as the takeover of Alcatraz Island in November of 1969. As Wilma Mankiller stated, "the Alcatraz experience nurtured a sense among us that anything was possible-even, perhaps, justice for native people” (as quoted by Wilkinson, 2005, p. 137). Another sign of the rising Indian was the establishment of the American Indian Movement (AIM), whose short-lived but dramatic tenure, including a temporary takeover of the BIA's headquarters, left an enduring impact of the psyche of the entire nation as it helped raise the consciousness of the general public regarding Indian concerns. This period also saw the establishment of the non-profit law firm, Native American Rights Fund (NARF), providing legal services for the protection and assertion of Indian rights.

Extra attention is given to these early years of the modern movement as they were critical for setting the tone of tribal governments turning the tide on literally centuries of erosion of their cultural traditions and sovereignty towards the new era of selfdetermination, which officially became U.S. policy in the 1970's. The significance of these events bore equal weight in the NW as elsewhere, and events in the NW also shaped the political and legal climate of the rest of the country.

In the Northwest we see the contexts of history and biogeography converge with the story of the salmon. The NW has been home to people whose ways of life revolved 
around the salmon runs, especially throughout the watersheds of the Columbia River and the Puget Sound areas. Over-harvesting from growing numbers of sport and commercial fishermen, habitat deterioration, and the introduction of dams throughout the region resulted in a devastating decline of the once great salmon runs. State policies were unfavorable towards Indian fishers, to put it lightly. For tribes who held treaties that ensured the right to hunt and fish at usual and accustomed locations there was a legal strategy that held promise. As treaties are federal law and through the U.S. Constitution override state law, tribes stood on solid legal grounds. This was confirmed through the Sohappy case (United States v. Oregon) which established Indian rights to a "fair share" of the fish harvest from the Columbia River. The Sohappy case also represented the first time the U.S. Justice Department stepped up to protect treaty rights against state law. The Boldt decision in United States $v$. Washington was a sweeping decision that reaffirmed tribal treaty rights, their sovereignty in regulating their own harvest, and interpreted their "fair share" as constituting a 50 percent allocation. The Boldt decision and other rulings in favor of tribes remain as the cornerstone of preserving the future of Indian peoples in the region. These decisions have brought hope to the Salmonpeople of the NW because it has translated to meaningful change on the ground, allowing them to live a key aspect of their lives in a manner consistent with their cultural traditions.

The story of Alaska differs in a fundamental way from the story of the lower 48 and the specifics of the NW, as Wilkinson (2005) recounts. Alaska did not assume statehood until 1959 and lacked the history of legal relations with the U.S. government. Up to this point, Alaska hosted minimal development, and most Natives continued life in much the same as they had for thousands of years. Once Alaska became a state treaties were never signed. Natives filed land claims, yet questions surrounding tribal sovereignty and land-ownership were not given much attention by Congress in the early years of Alaska's statehood. Then in 1968 oil was discovered on the North Slope, and resolution of the uncertainty around land ownership became important to U.S. economic interests. The result was a unique policy called the Alaska Native Claims Settlement Act (ANCSA) of 1971, which awarded Alaska Natives 45 million acres of land, but not the right of sovereignty. Essentially, the act established Native corporations granting certain land-ownership rights to those corporations, and members of various tribes received shares in the corporations. As entities the corporations fell under state jurisdiction. Meanwhile, the new shareholders' identities as members of village-based tribes remained largely unchanged. The win of preserving a way of life tied to the land came at the cost of sovereignty. ANCSA contained some insidious elements that threatened Native hold on the 45 million acres awarded. However, over the years the tribes have successfully “smoothed some of the 1971's law's sharpest edges” (Wilkinson, 2005, p. 239), have been successful in expanding preferential rights of hunting and fishing, and have capitalized on the corporate model becoming a major player in the Alaskan economy. The strongest indicator of Alaskan Native success has been the preservation of all of the acreage originally established by ANCSA. The policy represented by ANCSA has not been repeated, and Alaska remains an anomaly in its legal framework.

The recent history for the First Nations of British Columbia differs in a fundamental way. Unique among the historical relationships between First Nations and Canadian provinces, B.C. refused to sign land deals with all but a few tribes. Since land claims were never resolved, the courts have ruled that the First Nations retain legitimate 
claims to their ancestral lands, many of which have been heavily developed and/or are under public and private non-native management. Treaty negotiations among federal, provincial and First Nation parties have been underway since 1993 in an attempt to resolve the disputes over land rights and First Nation sovereignty. A recent report released by the federal and B.C. auditors-general illustrated how the talks have been ineffectual, failing to result in a single treaty agreement at a cost of $\$ 1$ billion to date (Cernetig, 2006). Ongoing negotiations include only 57 of the provinces roughly 207 First Nations. At stake are access to resources and their management and the sovereignty of the First Nations, estimated to collectively amount to 20-40 billion dollar settlements.

\section{Today and towards the future}

Trends of the recent past offer some encouragement for the prospects of the future of Indian Nations and First Nations. This is particularly true in the states where sovereignty has been fairly well established legally and the 562 federally recognized tribes are maturing in the practice of exercising jurisdiction over their own affairs (another roughly 250 tribes exist without U.S. federal recognition, at least nine within the Pacific Northwest). Economic development has grown significantly, casinos only being the most glamorous and in a few cases the most lucrative example. One place where the upswing is witnessed is in the success of Alaska Native corporations. Surveys conducted in 2005 credited Native corporations as comprising seven of 10 of the largest revenue generators in Alaska, 35\% of the largest revenue generators among state-based business in the U.S, and 17 of the 100 largest employers in the state of Alaska are Native corporations (Bluemink, 2006).

While the trend across the region as a whole is an encouraging one towards revitalization many challenges remain. According to statistical measures, the indigenous population of the U.S. remains near the bottom on most every major indicator, persistent barriers to the vitality of the salmon runs remain in place (read as "dams"), anti-Indian sentiment persists in some circles, and a host of environmental threats continue to jeopardize the health and wellbeing of individual Native people and their culture.

The Native population in the U.S., including Alaska Natives, remains among the poorest in the country, according to 2005 U.S. Census data (DeNavas-Walt, Proctor \& Lee, 2006). Figures suggest that while income has risen and poverty largely stabilized, incomes and insurance coverage for Native Americans remained among the lowest in the nation, with poverty rates among the highest. Averaging data from the three years of 2003-2005, the median American Indian and Alaska Native incomes was \$33,627, compared to \$46,326 for the median U.S. household. Only African-American households had a lower median income at $\$ 31,140$. On health coverage, nearly 30 percent, or about 661,000 American Indians and Alaska Natives were uninsured. Only Hispanics had higher rates of being uninsured at 32.7 percent. The three-year average of data suggests that 25.3 percent of Native Americans are living in poverty, compared with 12.6 percent of the total U.S. population. The figure indicates a slight increase from the 24.3 percent reported in the previous Census report on income and poverty. In total, 25.3 percent translates to 537,000 American Indians and Alaska Natives living below the poverty line (DeNavas-Walt, Proctor \& Lee, 2006).

The 2006 report card issued on childhood poverty by Campaign 2000 painted a similar socio-economic picture for the First Nation children relative to the broader 
population. Based upon data compiled by Statistics Canada, the report indicated that one in four First Nation children lived in poverty compared to one in six of all children. Compared to other Canadian children, First Nation children are more likely to suffer from health problems, have a doubled rate of disability, and live in overcrowded homes double the normal rate. Over half of First Nation homes contained mold contaminants, half of children under 15 live in single parent homes, and 40\% of off-reserve children live in poverty (Campaign 2000, 2006). Across the country, viewing the population as a whole the province of B.C. had the highest poverty rates at 23.5\%. The report did not indicate whether poverty was also disproportionately high in the 198 First Nations within B.C.

Present day Native communities also appear to be disproportionately impacted by many of ecological threats such as pollution and global warming (e.g., Houser et al., 1999). This exposure stems from lifestyles that are relatively more subsistence based. The disproportionate impact of these environmental threats to Native peoples illustrates concerns about environmental justice-recognition of the right of all segments of society, in terms to ethnicity, race and socio-economic status, to a healthy environment (see Camacho, 1998; Hofrichter, 1993). Discussing their review of 16 studies, Mohai and Bryant (1992) conclude that the findings "indicate clear and unequivocal class and racial biases in the distribution of environmental hazards” (Mohai \& Bryant, 1992). One example has emerged amid recent advances in understanding about the harmful effects of mercury, and the presence of increased concentrations of mercury in aquatic life (Commission on Life Sciences, 2000). As a population highly dependent upon fish to meet basic nutritional needs - to say nothing of cultural and spiritual needs-indigenous people appear to have higher concentrations of mercury in their blood and may have heightened health risks (Neumann, Kauffman \& Gilroy, 1997; Ringquist, 2000). Wheatley (1997) argues that even if the physical health risks are minimal, the presence of pollutants like mercury in fish has indirect detrimental impact on health and culture. Like toxicity of fish, global warming is another environmental threat that may have earlier and more dramatic effects on tribal communities due to lifestyles more closely tied directly with the natural environment (see Houser et al., 2004)

With the above biogeographic, historic, legal, and demographic context in mind, this project turns an eye towards the future. Recall the metaphor of the biological synthesis of space, time, and form offered by Croizat (1964), and the idea that "organic life is a constantly changing process expressed simultaneously across geographic space and on the time line of history" (p. 99). As organic life, we are caught in the midst of this process. While there are no facts about the future, possibilities of the future can be conceived, and within limits the landscape of future can and will be shaped through human choice and action. The methodology discussed next will allow exploration of these possibilities, and provide insight on the thinking of contemporary indigenous leaders regarding the meaning of sustainability, what a sustainable future might look like, and how to get there. 


\section{CHAPTER V}

\section{METHODS}

\section{Overview of Research Approach}

This project explored the mental models of sustainability held by indigenous leaders from the bioregion defined by the Pacific Salmon runs. Towards a contextual and generative contribution (Ritchie, 2003), a semi-structured interviewing strategy was employed, drawing inspiration from Ethnographic Futures Research as developed by Textor (1980, 1990a). Participants were selected based upon several criteria, including an established and respected history as a leader in their community with active engagement on sustainability issues. The interviews were audio-taped and/or videotaped and subsequently transcribed. Content of the interviews was analyzed using an iterative thematic coding approach as outlined by Ritchie, Spencer and O’Connor (2003), with support from the software program ATLAS.ti. The thematic coding served the foundation towards organization into a synthesized narrative representing the participant's perspectives. A primary goal of the content analysis was to identify elements of shared thinking among the various leaders representing diverse communities that are united by a relationship with salmon and an indigenous history in the bioregion. Notably divergent thoughts among participant's perspectives were also documented. Based upon this content analysis a narrative was composed summarizing the major themes and ideas expressed through the interviews. As a compliment to the narrative, several concept maps (Novak \& Gowin, 1984, Novak 1991) were developed to illustrate some primary aspects of the interviewees' mental models. The narrative and complimentary concept maps were shared with the participants who were invited to offer their feedback via an on-line survey as a measure of testimonial validity. Further details about the methods employed in the project are presented in this chapter, including a discussion of ethical considerations relevant to the research.

\section{Participants}

The sample of Native community leaders in this study was drawn from the bioregion defined by the Pacific Salmon Runs, including locations in Oregon, Washington, Alaska, and British Columbia, Canada. Many of the interview candidates were identified through their past and/or ongoing relationships with Ecotrust and the Buffett Awards, an annual award ceremony honoring indigenous leaders throughout the bioregion (visit www.ecotrust.org/Buffettaward/ for more information). The criteria for inclusion of participants included:

- Actively engaged in their community

- Respected track record in leadership roles

- Demonstrated knowledge of present day social, economic and environmental challenges

- Willingness and ability to commit the time to participate

Most participants satisfied the first three criteria as indicated by the interviewees' nomination for reception of and involvement with the Buffett Awards. Using the Buffett Awards for identification of potential participants was particularly appropriate for the criteria of being respected, as nominations come from within tribal communities and are 
reviewed and voted upon by tribal leaders. One participant was a member of the voting panel. Four of the participants from Alaska, all of whom hail from within the Copper River region, were not affiliated with the Buffett Award. These individuals were identified and recruited with the assistance of employees involved with Ecotrust's Copper River program and Gloria Stickwan, Subsistence Coordinator for Ahtna, Incorporated.

The sampling method employed is considered a purposeful or purposive sample as the participants were intentionally selected for the purposes of the research needs (Morse, 1991; Patton, 1990, 2002). Such a method is appropriate when it is necessary that the participants are knowledgeable informants of the research topic. Purposive sampling is not considered appropriate for most quantitative studies, but is valid for use in a qualitative study to provide depth and attain either hypothesized or unanticipated meaning (Berg, 1998). A purposeful sampling strategy is distinct from that of a convenience sample, as the former selects participants based upon their unique characteristics that constitute expertise in the area of study, and unlike the latter, selects participants without primary dependence on the ease and convenience for the researcher.

Recruitment of each participant was pursued via a direct phone call to introduce myself as the researcher and the general project idea. If the prospective participant expressed interest in learning more about the project, a follow-up e-mail was sent with several documents attached, including a project summary (see Appendix C), an outline of what their participation would entail (see Appendix D), a letter of informed consent (see Appendix E) and a letter of informed consent for having the interview videotaped (see Appendix F). Many prospective participants responded within a day or two to express their interest in participating. Those who did not respond immediately were contacted via phone within a week or two of the e-mail. If the prospective participant expressed interest in participating, then possible dates and locations for conducting the interview were explored and scheduled.

The project attained its high-end goal with a sample of 13 participants, well within the common range of 6-20 participants for qualitative studies involving in-depth interviews by sole researchers (J. Haaken, personal communication, February 6, 2007). Effort was made to ensure variability in age range and representation of both male and female genders, and representation across the bioregion. Ages of participants ranged from roughly 30 to 70 years of age. Nine women were actively recruited with five ultimately participating, and 10 men were actively recruited with 8 participating for a total participation rate of $68.4 \%$. Non-participation in four of the six cases was a function of incompatible availabilities (e.g., individuals were on vacation or overbooked at the time I was available to conduct an interview). A fifth case of non-participation was due to major health complications for the participant, and in the sixth case I simply never received responses to my phone calls and e-mails. Those who did participate were from across the region: five from Alaska, two from British Columbia, two from Washington, three from Oregon, and one from Northern California. Participants served within their communities in various, and sometimes multiple, leadership roles, including as democratically elected leaders, community activists, organizational leaders, and scholars. Table 3 provides a complete list of participants, their primary heritage, approximate residences at time of interview, and genders. Each participant was interviewed during the time frame of June 18, 2007 through January 3, 2008. 
Table 3

Participant names, primary heritages, residences at time of interview, and genders.

\begin{tabular}{llll}
\hline Name & Primary Heritage & Residence & Gender \\
\hline \hline Antone Minthorn & Cayuse & Umatilla Reservation (OR) & $\mathrm{M}$ \\
\hline Carol Craig & Yakama & Yakama Reservation (WA) & $\mathrm{F}$ \\
\hline Dennis Martinez & $\begin{array}{l}\text { O'odham / Anglo / } \\
\text { Chicano }\end{array}$ & Klamath Mountains (CA) & $\mathrm{M}$ \\
\hline Guujaaw & Haida & Haida Gwaii (off B.C. coast) & $\mathrm{M}$ \\
\hline Jeannette Armstrong & Syilx (Okanagan) & Okanagan Nation (B.C.) & $\mathrm{F}$ \\
\hline Joeneal Hicks & Ahtna & Ahtna Region (AK) & $\mathrm{M}$ \\
\hline Kathryn Martin & Ahtna & Ahtna Region (AK) & $\mathrm{F}$ \\
\hline Larry Merculieff & Aleut & Anchorage, AK & $\mathrm{M}$ \\
\hline Nicholas Jackson & Ahtna & Ahtna Region (AK) & $\mathrm{M}$ \\
\hline Nichole Maher & Tlingit & Portland, OR & $\mathrm{F}$ \\
\hline Roberta Conner & Cayuse & Umatilla Reservation (OR) & $\mathrm{F}$ \\
\hline Shawn Yanity & Stillaguamish & Arlington, WA & $\mathrm{M}$ \\
\hline Wilson Justin & Althsetnay & Ahtna Region (AK) & $\mathrm{M}$ \\
\hline
\end{tabular}

\section{Data Collection: Semi-structured interviews}

The semi-structured approach was chosen to allow the researcher "to gain a detailed picture of a respondent's beliefs about, or perceptions or accounts of, a particular topic" (Smith, 1995, p.9). As such, all the interview participants were asked a set of the same questions, but flexibility was retained to probe responses and pursue additional questions within each interview. Due to time limitations, two of the 13 interviews were unable to cover the entire interview script. The questions included in the interview script (see Appendix A) were mostly developed by the researcher and in consultation with members of the dissertation committee and Ecotrust's Director of Native programs. Aspects of the format and some specific questions were influenced by a variety of sources. The opening questions establishing the personal introduction were included to ensure cultural sensitivity (C. Jacobson, personal communication, November, 2006). The method of Ethnographic Futures Research (EFR) (Textor, 1980, 1990a, 1990b) inspired the idea of projecting into an imagined future. Based upon the recommendation of Smith and Anderson (2001), a question about what is not part of that future was included in recognition of the fact that a desirable future will be at least partially characterized by the absence of undesirable elements. The general outline of questions on an imagined future was influenced by the models of culture that move from observable to less visible dimensions (Schein, 1992, Triandis, 1972). In line with the perspective of Open Systems view (e.g., Katz \& Kahn, 1978) and Traditional Ecological Knowledge (Cajete, 2000) an emphasis was placed on questions about relationships. A series of "I am" statements was sought based upon an exercise described by Triandis (1994) for assessing self concepts (see Appendix G). Drawing from the structure generated through the EFR project with tribes in the Central and Owens Valleys of California by Gillis, Textor and Mitchell (2006), questions about specific actions for actualization of a particular future were included towards the end of the interview. 
The interview script was structured to establish a personal introduction of the interviewee and to tap three primary domains of interest: 1) conceptual meaning of sustainability; 2) a vision of a sustainable future; and 3) actions that must be engaged to bring the desirable future into reality. The reader can gain a better sense of these domains through review of the entire interview script as found in Appendix A. Due to time constraints two of the 13 interviews were unable to cover the full interview script. These two interviews focused on questions covering the personal introduction and the conceptual meaning of sustainability, plus basic coverage of actions and strategies. A brief synopsis of the typical interview is offered here.

Interview synopsis. After a brief welcome and introduction, the letters of informed consent were reviewed and signed. To open the interview, several personal questions were asked about the participant's family heritage and cultural identity. Questions then turned to the idea of sustainability, seeking to verify if this is an active term in the participant's vocabulary, leaving opportunity for other terms to be employed. In most instances a definition of the term was sought. In this portion of the interview, participants were asked about whether an image or symbol came to mind that represents the concept of sustainability. If yes, a request to draw the image and explain its meaning followed (paper and colored pens were immediately on hand for creation of the image). After a short break, interviewees were then asked to envision a future that embodies their concept of sustainability and to imagine themselves in that time and place. Participants were asked to explain what that scenario looks like, coaxed by specific questions and probing through the semi-structured interview format. Some of these questions focused on the imagined worldviews of the people that live in this future time and place. After a second short break, the final portion of the interview considered the actions that need to be engaged to realize the sustainable future. Two final questions were asked in closing, allowing the interviewee to: (1) include any other information they deem to be pertinent for inclusion, and (2) emphasize the key points/ideas from the conversation. The entire transcript is available for review in Appendix A.

Prior to the first formal interview conducted for data collection purposes, a pilot interview was conducted with Elizabeth Woody, Ecotrust's Director of Indigenous Leadership Program. The pilot helped to identify wording changes in the interview script to clarify the intended meaning of questions, and provided the opportunity to practice orchestrating the logistics of the interview process. The pilot also better prepared me for both the types of responses I might anticipate in the formal interviews, and how to most effectively lead the interview sessions, including prioritizing certain questions over others under time restrictions.

\section{Data Management \& Content Analysis}

Spencer, Ritchie and O’Connor (2003) outline qualitative data analysis as being comprised of two basic stages; data management and making sense through descriptive or explanatory accounts. Effective data management upfront lays essential groundwork and begins the sense making process. Huberman and Miles (1994) warn of the vulnerability of the qualitative researcher to become overwhelmed with unwieldy amounts of data if data management and analysis strategy are not well thought out in advance. Heeding the warning, thought has been put into how the interview data will be handled from start to finish, and an outline of the process in chronological order follows. 
Following the overall outline, further detail will be provided on the processes of familiarization, coding, verification, sorting, synthesizing, and the development of concept maps.

Overview. Following the completion of each interview, the recording was transcribed by an undergraduate research assistant, and then reviewed by myself to refine the quality of the transcription. Within two weeks of the interview the transcription was then delivered to the interviewee in electronic format. Interviewees were then invited to make edits as they deemed appropriate; such as, additions, clarifications, rewordings, or deletions. Upon return of the edited transcripts, the changes were immediately incorporated into the official and final draft of the transcript. This final draft was then uploaded into the software program ATLAS.ti (ATLAS), which served as the primary tool for data storage, indexing, retrieval, and analysis. Through initial review of the data, a preliminary indexing system was developed, followed by the time intensive activity of iterative coding of the transcripts (detailed further below).

Throughout the coding process themes were refined and linkages among the themes were identified and catalogued. Throughout the iterative coding and thematic mapping, a process of verification (similar to inter-rater reliability) was conducted to ensure my interpretation as the primary researcher was well grounded in the data. Where alternative interpretations were identified, negotiations among the verification team or adjustments to the coding were employed. After verification of the data interpretation, an attempt was made at articulating a synthesized narrative about sustainability from the perspectives of the participating indigenous leaders. As a final step, these leaders were provided with a copy of the synthesized narrative along with an evaluation form to assess testimonial validity, the degree to which they accept the narrative as reflective of their perspective (Stiles, 1993). With this outline in mind, the following pages detail key aspects of this process to assure clarity and transparency of the data management and content analysis strategies.

Familiarization. Familiarization entails developing fluency for the content area under study and the data obtained, and it begins the process of analysis by working towards identifying initial themes and concepts (Spencer et al., 2003). Familiarization began with conception and development of the research project and continued through a review of the collected data. Prior to conducting any interviews, the topic of focus was extensively researched, as reflected in the opening chapters of this document. Also, effort was made to obtain knowledge of the research participants' personal backgrounds, including reviewing biographic histories whenever available through on-line sources. During the interview process, familiarity was further developed with careful attention to the participants' responses. After interviews were conducted, reflection of the interview process and responses, then followed by reliving those responses while refining the transcripts solidified familiarity.

Thematic Index. One aim of the familiarization process, particularly during and after interview sessions, is to seek and document main themes and ideas. Identified themes were organized into an indexing system that represents major themes or categories and their sub-topics. Labels in the form of key words that capture the essence of the themes were generated for coding of the raw data. The index essentially provides the coding guide for the subsequent iterative review and coding of the interview 
transcripts. The index system that will be developed will be referred to as the General Thematic Index (GTI).

Coding. Working in ATLAS and with the GTI, iterative review of the transcriptions entailed coding, labeling or "tagging” portions of the text in accord with the themes they represent. This iterative review process constituted the core of the content analysis. The process involved careful consideration of each sentence and phrase of the transcripts while posing the question, "What is this about?" (Ritchie, Spencer \& O’Connor, 2003, p. 228). Two iterations of each interview were engaged by the principle investigator, with verification and necessary resolution through a research council (see below for verification process).

The process was approached in three segments that correspond to the three segments of the interview schedule: the concept of sustainability, visions of a sustainable future, and actions to achieve sustainability. The first segment focused on the opening portions of the interviews regarding each interviewee's thoughts on the term "sustainability." With the GTI in hand the iterative coding approach as outlined by Ritchie, Spencer and O’Connor (2003) was followed. The second segment of the coding process focused on the portion of the interview entailing exploration of visions of a sustainable future. This content was assessed via two iterations with the GTI.

Within the vision portion of the interviews, a series of "I am" statements were generated by the participants (see Appendix G). Following the guidelines of Triandis (1994), these statements were coded in terms of, individual level attribute, social or group affiliation, and ecological affiliation - the later code going beyond Triandis's guidelines. Ecological affiliations can entail reference of self in terms of natural elements (e.g., water), plant life, animal life, or geographic locations (e.g., a particular river valley). Starting with this coding scheme, two coders, the principle investigator and an undergraduate research assistant trained in Triandis's methodology, independently coded each statement. After an initial round of coding, discussion occurred around the issues encountered. In practice, the three category approach outlined above proved to be too constraining as many of the "I am" statements did not fit clearly into one category. As such, additional cross-cutting codes were used, such as individual/social (e.g., "I am speaking my language”), or social/ecological (e.g., "I am Alaskan Native”). After an additional round of independent coding, the two coders systematically compared their respective codes for each statement. In the few cases where differing codes were applied, discussion was engaged until an agreeable resolution of how to code the item was reached. The guiding principle towards seeking agreement was to stay close to the explicit statements and minimize presumptive inferences. This discussion generated a clearer understanding of the codes themselves, and subsequently led to recoding other items. Appendix G provides definitions of each code category, and a complete listing of the generated "I am" statements organized by code.

The final segment of the coding process focused on the actions needed to bring the optimistic vision of the future into reality. This initial strategy sought to catalogue identified actions into a list and code in terms of (a) the level at which the action is suggested to be initiated (e.g., individual, tribal, national), and (b) the level, population, or system the action is targeted to affect (e.g., national, students, or legal system). In practice, this approach proved to result in arbitrary decisions and assumptions by the researcher as many actions had no clear initiator or target. For example, identification of 
the importance of working together and coalition building without specific indicators of the parties involved. Subsequently, all occurrences of "actions/strategies" were coded as such, and those occurrences were then reviewed to identify major themes or categories of action types. The process of review for theme identification within actions/strategies was conducted by a student research assistant and myself. After our independent reviews, we compared notes which very closely mirrored one another. Dialogue primarily focused on resolving semantic labeling. After agreeing upon coding labels, the actions and strategies were formally coded within ATLAS along with the rest of the content.

Verification Process. To ensure that my interpretation of the interviews was grounded in the data, I convened regularly with a research council (J. Haaken, personal communication, February 13, 2007) throughout the data analysis and representation phases. The concept of a research council refers to consulting with both a group and with Native experts. The group consisted of myself as principle investigator, Dr. Jan Haaken, and three undergraduate research assistants. Individuals with Native expertise that were consulted with included members of the dissertation committee, members of the Ecotrust staff, as well as several of the research participants themselves. Within the group setting and individual conversations, the role of the research council was to provide oversight of my analysis of the interview content and facilitate reflection on the process. Further these conferences generally helped me to come to a deeper understanding of the interview content. On several occasions, lessons learned from these conversations with members of the research council required revisiting aspects of the data analysis to rework in a manner consistent with the new insights gained. For example, one research council member observed that the theme of nature as the context of life and a determining force was paramount across many of the interviews. This observation drew my attention to the fact that my current coding scheme was not directly capturing this theme. The code "natural law" was created, and previously coded transcripts were revisited to identify occurrences of this theme.

Sorting. After thorough coding of the themes of the interviews, the themes were sorted to bring similar content together, enabling the researcher an opportunity for deeper consideration of the meaning and substance of that theme (Ritchie, Spencer \& O'Connor, 2003). It is worth noting that throughout the iterative review, the same sections of material may have been coded with multiple labels and subsequently sorted according to the multiple themes. ATLAS enabled this sorting without having to carve up multiplelabeled sections or losing connection of any of the material from its original context.

Establishing Shared and Divergent. The challenge of identifying elements of shared and divergent thinking was attended to throughout the research process. After each interview the content was given cursory review and held in mind during subsequent interviews, where targeted probes were occasionally used to verify if the present participant shared perspective with earlier participants (E. Mankowski, 2007, personal communication). For example, one question was posed, "Another thing that has come up, I'm wondering if you see it this way too, is the connection between sustainability and health. Is that a meaningful connection for you?” Further, after the coding and sorting processes were engaged, the various themes were viewed with greater scrutiny. Segments representative of a given theme were compared across interviews to determine whether the theme was addressed by other interviewees and whether those other interviewees discussed the theme in similar or contradictory terms. If multiple 
participants discussed a theme in similar terms, without dissenting perspectives, then that theme in those terms was considered for inclusion in the narrative as a shared line of thought. Differences among perspectives were noted and discussed in terms of the nature of the divergence. Perhaps due to the extensive breadth of the topic, most differences in perspectives were found to be complimentary rather than contradictory. For example, some participants placed greater emphasis on the importance of youth education, while other participants emphasized political change and/or community reorganization. While different, both have legitimacy and are not inherently in conflict with one another. Finally, the testimonial validity process discussed below provided participants with the opportunity to express agreement or disagreement with specific ideas and the overall results in general.

Synthesis. The next stage of the analysis process entailed a summarization of each of the themes and their key relationships. This effectively reduced the amount of material that needed to be handled and began to give clarity to the essence of the data (Ritchie et al., 2003). Through this process, I followed the advice of Ritchie, et al (2003) and preserved the interviewee's language as much as possible, including general terminology and usage of exemplar quotes. The thematic synthesizing sought to retain the coherence and integrity of the major concepts shared by the participants. The thematic summaries provided the cornerstone of the comprehensive narrative summary. In addition to summaries of the participants' identities and community affiliations, the narrative was organized into three major sections summarizing: (a) the concept of sustainability; (b) a vision of a sustainable future; and, (c) the actions for realizing the sustainable future. These categorical summaries seek to provide readers with a holistic understanding of the totality of the material obtained (see also Representation below).

The decision was made to compose the results section in narrative form, using a first person plural, or "we" narrative voice. This decision was made in part because of the goal for the findings to be accessible to a broad audience, and to preserve the tone of the interviewees who spoke in first person and first person plural in the interpersonal format of the interviews. More is stated about the use of the "we" narrative voice in the Results section.

Concept Maps. Throughout the process of developing a synthesized document reflecting the shared thinking among the interviewees, I developed several complimentary diagrams to illustrate key aspects of the perspectives shared. The diagrams I created are in the same general vein as influence diagrams (e.g., Howard \& Matheson, 1981), cognitive maps (Tolman, 1948), concept maps (Novak \& Gowin, 1984, Novak 1991), mind maps (Buzan, 1991) and causal-loop diagrams (e.g., Sterman, 2000). Each of these terms reflects the same basic notion of mapping out patterns of relationships among identifiable elements, variables, or concepts. Speaking specifically to causal loop diagrams Sterman (2000) notes that they are useful tools for capturing and communicating the mental models of individuals (or teams) regarding the dynamics of a system, particularly the feedback cycles one believes is central to explaining a phenomenon. Such visual representation seeks to aid in communicating the pattern of relationships and complex processes identified by the study in a manner distinct from traditional text representation-effectively bringing the findings alive in a different manner (White, Woodfield \& Ritchie, 2003) and making those findings more accessible to a broader audience (Novak, 1991). 
Each of the mapping terms (e.g., concept maps, mind maps) arises from different disciplines and each provides varying guidelines for application and varying degrees of formality. In my application, I chose to use the term concept map because it consistently refers to the representation of knowledge structures or patterns of thought in graphical form, and its application is flexible enough to permit unique representational forms that best capture the thought patterns being mapped (e.g., Novak, 1991). The maps were developed through review of the major themes and concepts identified by the analysis process and the explicated relationships with other themes and concepts. Specific use of icons and symbols followed the symbol key outlined in Figure 7. The concept maps developed through this project are not intended as an analytic interpretation, rather simply as a descriptive mapping of that which is made clear by the interviewees. They serve as a graphic depiction of aspects of the narrative content as derived from the interviews.

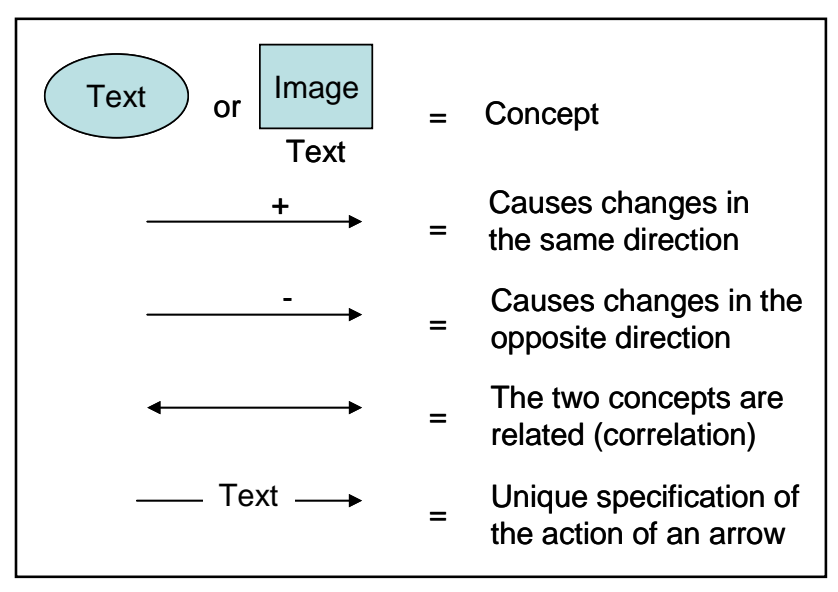

Figure 7. Concept Map Symbol Key

\section{Determination of Quality}

The use of a qualitative methodology demands consideration of what determines quality in the given project. A positivist position would assert that the merits of qualitative research should be assessed via the same standards as quantitative research, namely the criteria of internal and external validity and reliability and objectivity. Taking a post-positivist stance, I will strive to meet the challenges of trustworthiness and representation as described by Merrick (1999).

First, I will explain why the common standards of reliability and validity as traditionally employed are not appropriate for this project. The postpositivist perspective rejects the idea that there is one "truth" and recognizes that knowledge is constructed, thus "the aim (and even the possibility) of replication is thrown out" (Merrick, 1999, p. 28). Qualitative researchers generally agree that even the same researcher would not be able to replicate a study given the inherently unique, personal, and contextually dependent nature of the research process (Banister et al., 1994). In terms of validity, the importance of adequately capturing the phenomenon of interests is retained. But, in qualitative research it is not about pinpointing the truth and facts as they exist in some objective world. Rather, validity is a measure of "the adequacy of the researcher to understand and represent people’s meaning” (Banister et al., 1994, p. 143). The 
knowledge obtained and truth of the knowledge is contextually contained, with understanding by the researcher, readers and participants being the aim (Merrick, 1999).

Trustworthiness. Discounting the criteria of objectivity, reliability, and traditional meanings of validity, the alternative term of trustworthiness is suggested to inform whether "good practice" was present throughout the research (Stiles, 1993). Stiles reviews the following means of ensuring trustworthiness: (a) openness of the researcher's orientation, (b) intensive and prolonged engagement with the subject matter (c) persistent observation, (d) triangulation of data collection, and (e) discussion of methods and findings with informed others, as well as (f) iterative engagement between observation and interpretation to ensure grounding of the interpretations in the data. Merrick (1999) summarizes that trustworthiness is a matter of "how one approaches, collects, analyzes, interprets, and reports the data” (p. 31), with importance in making the steps engaged and possible influences transparent to the reader. Regarding (a), recognition of the researcher's intimate role in constructing the research agenda and subsequent data collection and analysis calls for making explicit the researcher's orientation and potential bias. In this spirit, I provided personal background that set the stage for my engagement in this project in the preface, “Author's Perspective." "Having [the researchers'] orientation in mind, whether or not we share it, helps us put their interpretations in perspective” (Stiles, 1993, p. 602).

Elements (b) and (c) of trustworthiness were satisfied by the intensity of the research methods, and the necessity to meet the rigorous institutional standards as a dissertation research project. Element (d), triangulation, was not formally pursued as part of the effort to ensure trustworthiness. Element (e) was satisfied through periodic engagement with members of the dissertation committee, members of the supporting organization Ecotrust, and the research council consisting of Dr. Jan Haaken, other graduate students, and undergraduate research assistants. The final element of Stiles' outline for trustworthiness, the iteration between observation and interpretation, was practiced throughout the research process. For example, after each interview the content was considered and reflected upon, in multiple instances giving influence to specific probes of the following interview to test whether an appropriate understanding was being developed.

Documentation. Throughout data collection, analysis and representation I sought to document the research process. I maintained a notebook and the use of ATLAS helped to ensure traceability of my data collection to the final results of my project. This is sometimes referred to as maintaining a "chain of evidence" such that someone reviewing the project can follow how the final results were derived from the data collected (e.g., Paré, 2002). The notebook was used, particularly during the data collection phase, to record the types of decisions that were made, and personal notes reflecting on points of insight or changes in my thinking. The notebook serves as a record of my experiences in the field collecting data (see Appendix $\mathrm{H}$ for a sample notebook entry). The "Memo" feature of ATLAS facilitated recording of my thoughts while interacting with the data during content analysis and representation. Additionally, the functionality of ATLAS enabled preserving links between original data sources and the various stages of data analysis. Together the notebook and the features of ATLAS help provide documentation of a chain of evidence. 
Representation. The ultimate aim was to produce a study that adequately reflects participants' perspectives about sustainability, and represents these perspectives in a clear and accessible manner. Towards this end, several steps were taken. First, effort was made throughout the study to minimize the power dynamics that often occur in research when the researcher is cast as expert and participant as subject (Roberts, 1981). Rather, this project was engaged with effort to establish the participant as expert, and the researcher as listener or learner. These efforts are important as much for the participant to feel respected and more inclined to share openly, as for the researcher to maintain a humble disposition that is receptive to the participant's perspective with minimal imposition or projection of the researcher's perspective during the interview session, as well as during later interpretation.

To further protect against projection and towards generally representing participant's perspectives accurately, effort was given to establishing testimonial validity - the accuracy of the research as determined by the informants themselves (Stiles, 1993), also know as member checks (e.g., Patton, 1999). After each interview took place, interviewees were provided a copy of the interview transcript within two weeks for their review. If the interviewee determined that any portion of the transcript misrepresented their perspective they had the opportunity to remove or alter it however they saw fit. They also had the opportunity to add or clarify thoughts as they saw appropriate. Participants varied significantly in the degree to which they modified their transcript. Some made only cursory edits, such as spelling corrections requested by the researcher, while others made significant modifications, including complete removal of passages. The revised transcripts were then used in the subsequent content analysis.

Testimonial validity was also sought after the content analysis and representation was completed. Once the synthesis of the interviews was produced a copy was provided to each interviewee along with an on-line survey link. The survey, developed based upon the construct description of testimonial validity found in the literature, provided an opportunity for each interviewee to rate how well the synthesis represents their perspective and the degree to which they endorse the document (see Appendix I). The Results chapter includes a summary of the testimonial validity survey results. The attention to testimonial validity also served to protect from some potential ethical concerns, which I now review more broadly.

\section{Ethical Considerations}

Being sensitive to both the potentially emotionally charged elements of the subject matter and the intimate nature of qualitative methodology, this project was approached with attention to ethical considerations as they may arise before, during and following the research endeavor (Cieurzo \& Keitel, 1999). Great care was exercised throughout the research process to ensure transparency of the research agenda, safeguards for participants rights and well-being including protection of their community's privileged information, and reciprocity in the research relationship.

Transparency of the research agenda. Early in the recruitment phase, potential participants were provided with a comprehensive outline of the research agenda including, the interview script and description of the anticipated deliverables (see Appendix C). Contact information was provided and those considering participation were encouraged to ask questions and have concerns addressed before committing to the 
project. An open line of communication was maintained with each participant throughout the entire project.

Participants' rights and well-being. Before any data were collected the project was subjected to Human Subjects review. All fundamental elements, including the strategies for recruitment, obtaining informed consent, reasonably ensuring confidentiality and anonymity, protection from harm, and concerns of deception were addressed. A couple of these elements are worth giving direct attention to. Within the context of the interview, subjects may have provided statements that revealed confidential or private information. As described above, upon completion of each interview a transcript was created and sent to the participant for his or her review. If a participant decided that he or she would like aspects of the transcript omitted or altered, they were given the freedom to make such changes.

Beyond the inclusion or exclusion of specific content, caution was demanded in the reporting phase where the researcher was in the position of relaying the perspective of the participants. This created a challenge for the researcher to responsibly balance "the illusion of objectivity and the borders of subjectivity" (Fine, 1994, p. 75). This challenge was of particular relevance here due to the cultural differences between researcher and participants. Awareness of this responsibility, oversight from the dissertation committee, transparency of the methodology employed, and the testimonial validity process served to protect against this concern.

Reciprocity. Whatever value produced by this project is owed to the willingness of the interviewees to participate and share their perspectives on the subject matter. Reciprocity is essential to ensure that all participants feel that their time has been respected and valued, and to ensure they experience some tangible benefit in return. For one, a modest gift, such as some tribal caught salmon, was given to each participant at or shortly after the time of their interview as a token of appreciation. Further, all participants were provided copies of the final materials produced. If any other publication materials emerge from the effort, participants will be provided copies and consulted about appropriateness of content. Additionally, the research was conducted with a general commitment to taking the interviewee's message and relaying it to Native and non-Native audiences in the name of expanding awareness of indigenous perspectives on sustainability. Towards this end, a web-site was developed (see Appendix K) that serves as a public space where anyone is able to learn from this project's inquiry of Native perspectives on sustainability. Additionally, to date, two video pieces featuring two of the interviews have been produced and aired on public access television in the greater Portland area (see Appendix L). 


\section{CHAPTER VI}

\section{RESULTS}

This chapter presents the results of in-depth interviews on the subject of sustainability with thirteen respected Native leaders from within the bioregion defined by the Pacific Salmon Runs of North America (Salmon Nation). The chapter's opening paragraphs provide general orientation to the results as summarized in narrative form. Documentation of the results from the testimonial validity process is then presented, followed by the actual narrative summary.

I approached this project as a student seeking to learn. I listened carefully to what was shared with me, and this narrative summary is my best attempt to reflect back what I heard and learned through my own set of perceptual filters (see "Preface: Author's Perspective" above). Representing the depth and detail of each interview is impossible, so what the reader will find here reflects main themes of those interviews, particularly themes that were expressed across multiple interviews (each interview can be found in its entirety at www.nativeperspectives.net). There are no ideas included in this narrative that were not communicated by at least one of the interviewees. While each idea was not expressed by every participant, the decision was made to use a first person plural, or "we" voice as the most effective way to synthesize all perspectives into one unified narrative. Effort has been made to avoid suggestions of homogeneity among participants by acknowledging the diversity of participants (see "Who we are" below), and when thoughts did significantly differ on a particular topic to make that apparent to the reader. The "we" voice is found in other works with similar purposes (e.g., Gillis, Textor \& Mitchell, 2006; Ladum, 2006), and is also consistent with the language frequently used by most of the participants when speaking from a place of identification with their community, indigenous peoples, and/or all of humanity. One participant did express reservations about the use of the "we" voice, which is discussed in "Testimonial Validity Survey Results” below (see also Appendix I and Appendix J).

By integrating the multiple perspectives of all participants into one narrative, the extensive topic of sustainability is more thoroughly addressed. Each community represented by the participating leaders faces similar yet unique challenges. Specific ideas and actions expressed in the narrative may be more or less relevant depending on the specific historical, ecological, political and legal contexts of each respective community. Hopefully, this narrative articulates something of relevance to all communities, directly represented or not.

This narrative is intended to serve as a contribution to the conversation on sustainability and to encourage a more extensive and inclusive dialogue around the longterm interests of all people. I remind the reader that I assume no authoritative voice on the subject of Native perspectives on sustainability. All readers are invited to be the judge of this narrative. The extent to which its content rings true, yields insight, and/or provides direction for each individual is the true measure of its validity.

The structure of the narrative results section begins with brief statements of the identities of the participating leaders ("Who We Are") and the communities with which they identify ("Our Communities"). This is followed by discussion around the use of the term "sustainability” and its meaning ("'Sustainability:' The word and concept”). Next is 
a vision statement illustrating core elements of the participants' hopeful vision of a sustainable future for their communities (“Our Vision”). As recommended by Levin (2000), the vision is composed in a present tense storytelling format to more effectively transport the reader's imagination to the desired future. The final element of the narrative is a summary of the kinds of actions and strategies identified by the participants as critical to achieving such a future ("How We Get There"). Throughout the narrative, endnotes are used to indicate which interviews provided the basis for the associated content. Where direct quotes are included, the first cited number corresponds to the interview directly quoted, and each following number references another interview where a very similar thought was shared. The endnote list appears immediately following the narrative. Prior to presentation of the narrative, the results of the testimonial validity survey are presented to inform the reader of the degree to which the participants themselves found the narrative to be an accurate representation of their perspectives.

\section{Testimonial Validity Survey Results}

Participants were provided with copies of the narrative results and invited to complete an on-line survey to facilitate their feedback and provide assessment of testimonial validity, the degree to which the narrative was viewed as an accurate representation of their respective perspectives on sustainability (Stiles, 1993). Ten of the thirteen participants responded to the survey (see Appendix I) for complete response data). An eleventh participants offered e-mail correspondence and indicated general satisfaction with the narrative. Feedback via the survey was largely positive, with minor issues identified by several participants and major issues identified by one participant (e.g., see Table 4). Questions about how well the narrative included a given participant's perspective and how well the overall narrative represented their perspective in general were scored from 0.0 to 5.0, with 0.0 corresponding to "strongly agree" and 5.0 corresponding to "strongly disagree." Mean scores ranged from 0.6 to 1.0, and standard deviations ranged from 0.52 to 1.12 , indicating an overall solid level of agreement with the content of the narrative with some variability among the participants (see Table 5). Additional open-ended questions were posed to ensure accuracy in representation and to further gauge participant reaction. Appendix I provides full documentation of all responses to the testimonial validity survey. 
Table 4

Participant responses to survey question assessing their comfort with their name being associated with the narrative as originally composed.

\begin{tabular}{|c|c|}
\hline Name* & $\begin{array}{l}\text { Response option selected to question: } \\
\text { "Which of the following statements best reflects your position?" ** }\end{array}$ \\
\hline Antone Minthorn (Cayuse) & $\begin{array}{l}\text { "I am comfortable with my name being associated with this } \\
\text { narrative as it is." }\end{array}$ \\
\hline Carol Craig (Yakama) & $\begin{array}{l}\text { "I am comfortable with my name being associated with this } \\
\text { narrative as it is." }\end{array}$ \\
\hline $\begin{array}{l}\text { Dennis Martinez (O'odham } \\
\text { / Anglo / Chicano) }\end{array}$ & $\begin{array}{l}\text { "I am comfortable with my name being associated with this } \\
\text { narrative as it is." }\end{array}$ \\
\hline $\begin{array}{l}\text { Jeannette Armstrong } \\
\text { (Syilx) }\end{array}$ & $\begin{array}{l}\text { E-mail correspondence: "I was fine in general with the } \\
\text { statements. Good work." (personal communication, April 30, 2008) }\end{array}$ \\
\hline Joeneal Hicks (Ahtna) & $\begin{array}{l}\text { "I am comfortable with my name being associated with this } \\
\text { narrative as it is." }\end{array}$ \\
\hline Kathryn Martin (Ahtna) & $\begin{array}{l}\text { "I am comfortable with my name being associated with this } \\
\text { narrative as it is." }\end{array}$ \\
\hline Larry Merculieff (Aleut) & $\begin{array}{l}\text { "It would require significant changes for me to be comfortable with } \\
\text { my name being associated with this narrative." }\end{array}$ \\
\hline Nichole Maher (Tlingit) & $\begin{array}{l}\text { "I am comfortable with my name being associated with this } \\
\text { narrative as it is." }\end{array}$ \\
\hline Roberta Conner (Cayuse) & $\begin{array}{l}\text { "I am comfortable with my name being associated with this } \\
\text { narrative as it is." }\end{array}$ \\
\hline $\begin{array}{l}\text { Shawn Yanity } \\
\text { (Stillaguamish) }\end{array}$ & $\begin{array}{l}\text { "I am comfortable with my name being associated with this } \\
\text { narrative as it is." }\end{array}$ \\
\hline Wilson Justin (Althsetnay) & $\begin{array}{l}\text { "With a few minor changes, I would be comfortable with my name } \\
\text { being associated with this narrative." }\end{array}$ \\
\hline $\begin{array}{l}\text { * Two participants, Nichol } \\
\text { ** Response options not se } \\
\text { a) "I am not comf } \\
\text { b) "Other (please }\end{array}$ & $\begin{array}{l}\text { Jackson and Guujaaw, did not complete the survey. } \\
\text { ected by any participants: } \\
\text { rtable with my name being associated with this narrative.” } \\
\text { becify)” }\end{array}$ \\
\hline
\end{tabular}


Table 5

Response distributions to selected items from the testimonial validity survey (see Appendix I for complete documentation of the survey's items and responses).

\begin{tabular}{|c|c|c|c|c|c|c|c|c|}
\hline Item & St A & $A$ & So $A$ & So $D$ & $D$ & St D & Mean* & $S D^{*}$ \\
\hline $\begin{array}{l}\text { My perspective is represented } \\
\text { in this narrative. }\end{array}$ & 3 & 5 & 2 & 0 & 0 & 0 & 0.9 & 0.74 \\
\hline $\begin{array}{l}\text { The key aspects of my } \\
\text { thinking on sustainability are } \\
\text { included in this narrative. }\end{array}$ & 4 & 6 & 0 & 0 & 0 & 0 & 0.6 & 0.52 \\
\hline $\begin{array}{l}\text { As a whole, this narrative } \\
\text { reflects my thoughts on the } \\
\text { subject of sustainability. }\end{array}$ & 4 & 4 & 0 & 2 & 0 & 0 & 1.0 & 1.16 \\
\hline $\begin{array}{l}\text { I'd feel comfortable presenting } \\
\text { this narrative to my peers as } \\
\text { reflective of my perspective. }\end{array}$ & 3 & 5 & 1 & 1 & 0 & 0 & 1.0 & 0.94 \\
\hline $\begin{array}{c}* \text { Means and Standard Deviation } \\
\text { St } A=\text { Strongly Agree }(v \\
A=\text { Agree (value = }) \\
\text { So } A=\text { Somewhat Agree } \\
\text { So } D=\text { Somewhat Disag } \\
D=\text { Disagree (value }=4 \\
\text { St } D=\text { Strongly Disagre }\end{array}$ & $\begin{array}{l}\text { calcul } \\
\text { lue }= \\
\text { (value } \\
\text { ree (va } \\
\text { (valu }\end{array}$ & & & & & & & \\
\hline
\end{tabular}

Greatest reservation about the narrative was expressed by Larry Merculieff (Aleut). A phone conversation and a series of e-mail exchanges were engaged to better understand the issues of concern for acknowledgement here. Appendix J offers correspondence shared by Merculieff regarding his primary concerns with the format of the narrative. Merculieff found the "we" narrative voice problematic due to its implication of grouping all participants in consensus around the statements. Concern was also expressed about taking information and quotes out of the full context that they were originally shared. This was cited as a recurrent problem in the attempts to "incorporate" traditional ways of knowing into Western science (L. Merculieff, personal communication, April 13, 2008). Merculieff also identified use of some problematic language. The handling of these issues and others raised are addressed below.

Based upon all the feedback received, and after consulting with members of the project's research council, several changes were made to the final narrative, and several elements left the same. These are as follows:

(1) In response to Merculieff's expressed preference to not have his name associated with the narrative content, all citations linking narrative statements to his interview content were removed. Two direct quotes of his were removed and replaced with paraphrased versions. Replacing with paraphrased content was essential to maintain the completeness of the narrative as reviewed and supported by the other participants. The two paraphrased sentences are: "In our worldview, everything is profoundly connected," and, "We must embody our awareness of the intimate and profound connection among all things. We possess an inherent intelligence, and if we operate with our minds and our hearts connected, then we will move in greater harmony with creation.” 
(2) All occurrences of the phrase "natural resources" were replaced due to expressed concerns that it was Western terminology and predisposes fish, wildlife and habitats to be viewed as commodities.

(3) Under the section, "A Human Focus," the occurrences of the phrases "people centered" and "human centered" were replaced with the phrase "a human focus." Concern was expressed that "human centered" implied that humans are more important than the Earth. Such an implication would be inconsistent with other themes of the narrative. The phrase "a human focus" was also deemed to be more consistent with the content of the original interviews. An additional modification to sentence order was made to give greater clarity to the intended meaning of the section that more focus needs to be given towards understanding our nature as human beings and towards structuring human systems to be in greater alignment with the natural world.

(4) The narrative's concluding statement initially read: "Gifted with this potential, our purpose is to continuously maintain ourselves and all our relations in harmony and balance with natural law. The extent to which our cultures serve this purpose is the extent to which they are ultimately valid and worthy of being passed along to future generations." The words "valid and” were removed due to concerns expressed about "valid" being a loaded term. Beyond loaded meaning, there was concern that with use of the word "valid," particular aspects of a culture that do not directly serve the stated purpose could be deemed as invalid. It was decided that the intended meaning of the statement was retained without the term.

(5) The first person plural ("we") voice was retained due to the support expressed by the vast majority of the participants, including direct statements of appreciation for use of the collective voice. The practicalities of restructuring were also a significant deterrent. I also personally believe that the accessibility, readability, and overall appeal of the content are enhanced by the narrative in its current form (see Appendix $\mathrm{J}$ for critique of this representation decision).

(6) Regarding the concern about quoting out of context, this is acknowledged as an inherent limitation of any exercise that necessitates reduction of information. As noted in the opening to the results section, readers are directed to the project website (see Appendix K) for complete interview transcripts. Beyond this, the testimonial validity survey was designed to reduce the problem by giving participants the opportunity to identify misrepresentations of any of their statements or perspectives. No specific statements were cited as being misrepresentative. The general concern led to the removal of two direct quotes as detailed above.

(7) A comment that the narrative lacks coverage of leadership as a key element in sustainability prompted consideration of the topic's inclusion. Leadership was raised as a topic under “Our Vision” and "How We Get There,” but not directly under “'Sustainability:' The word and concept.” Reviewing original content from the interviews, it was concluded that leadership was covered consistent with the manner in which it was spoken to by the participants as a whole-at least as interpreted by the researcher. Justin, who made the comment, did speak at greater length about leadership than many other participants. Indeed, his perspective was highly influential in prompting as much coverage of leadership as is found in the narrative. The reader is encouraged to see the full interview of Justin for more depth on the topic of leadership. 
(8) "Recognizing God for giving us this gift," was shared as a comment related to the survey's question about level of agreement with the concluding statement of the narrative. The comment is acknowledged as legitimate, but it was not added as it would significantly alter the concluding statement as evaluated by other participants. Also, recognition of the Creator as the source of gifts appears several other times throughout the narrative.

(9) Despite the suggestion for removal, use of the term "sustainability" was retained throughout to fulfill a key aspect of the project's intention to explore the conceptual meaning of "sustainability."

The testimonial validity process in general helped to verify and refine the accuracy of my representation of the perspectives shared. The updated narrative summary follows. 


\section{Narrative Summary}

Who We Are

We are Native leaders within the region defined by the Pacific Salmon runs of North America. We are women and we are men with similarities and differences. Our backgrounds vary from having received a traditional upbringing to having been raised apart from our traditional culture, only to return to it later in our life. Our ancestral heritages are diverse, with deep Native roots, as well as European links (see Table 6 for a listing of the heritages with which we are related). We are a mix of democratically elected leaders, community activists, organizational leaders, and scholars. We are all dedicated to protecting, preserving and restoring our Native cultures and the planet.

Table 6

Personal heritages cited by participants

\begin{tabular}{|l|l|l|}
\hline Ahtna & Irish & Spanish \\
\hline Aleut & Muckleshoot & Squaxin \\
\hline Althsetnay & Nez Perce & Stillaguamish \\
\hline Athabascan & Norwegian & Swedish \\
\hline Cayuse & O'odham & Syilx (Okanagan) \\
\hline Chicano & Portuguese & Tlingit \\
\hline French & Puyallup & Umatilla \\
\hline German & Russian & Yakama \\
\hline Haida & Snohomish & \\
\hline
\end{tabular}




\section{Our Communities}

Like us as individuals, our communities differ. Some are more urban while most are more rural. Legal rights vary dramatically from full sovereignty to no formal recognition. Some of our communities have an extensive land base, others have parcels of scattered land, while some have little or no land. In no case does the land base of our communities today reflect our historical land base prior to European contact. The places we call home vary from the Aleutian Islands of the Northern Pacific Ocean, to lush temperate rainforests, to the dry, arid lands east of the Cascade Mountains. Some of our communities are not defined by place, but rather by shared traditions or heart to heart connections with others around the globe. For many of us, our communities include the plants and animals with whom we share the land and have intimate relationships, both historically and present day. Many of those relationships, and our communities in general, have been disrupted and are threatened by interrelated factors including, resource exploitation, deterioration of the local landscape from encroaching development, species extinctions and population declines, a rapidly changing climate, and deterioration of our cultural traditions, including the loss of our Native languages and traditional knowledge systems. In the midst of this ecological and cultural crisis, we offer our thoughts on the meaning of the term "sustainability," followed by an expression of our hopeful vision for the future of our communities, as well as some of the actions and strategies needed to achieve our vision. 
"Sustainability:" The word and concept

The term "sustainability" is being used more and more frequently as greater attention is being giving to a broad set of problems for which the human species is responsible. Most of us use the word "sustainability" to some extent, while just less than half of us regularly use the word (see Table 7). In using and hearing the term, most of us hold some reservations about how it is applied. We recognize that many people, particularly in industry, use the term to serve their own interests without seriously considering its meaning and the context within which it is applied. ${ }^{5,11,13}$ By itself, sustainability is a very abstract term, thus it needs to be broken down a bit to understand its meaning. ${ }^{13}$ For starters, Table 8 provides some definitions and initial thoughts about the word itself. Further, Figure 8 illustrates some of the key topics that come to mind when we think of sustainability. As indicated by this figure, sustainability is linked with many other things. In other words, the issue of sustainability is tied up with pretty much everything we could talk about. ${ }^{2,11,13}$

Table 7

Participant use of the term "sustainability"

\begin{tabular}{lcccc}
\hline \multicolumn{1}{c}{ "Sustainability” the term } & $\begin{array}{c}\text { Use } \\
\text { regularly }\end{array}$ & $\begin{array}{c}\text { Use, but not } \\
\text { regularly }\end{array}$ & $\begin{array}{c}\text { Use rarely if } \\
\text { at all }\end{array}$ & $\begin{array}{c}\text { Use with } \\
\text { reservation/ } \\
\text { qualification }\end{array}$ \\
\hline \hline Participant use of term & $46 \%$ & $38 \%$ & $15 \%$ & $54 \%$ \\
\hline Closely associated terms: & & & \\
\hline $\begin{array}{l}\text { Sustenance, conservation, preservation, taking care, protecting, respect, humility, cultural } \\
\text { sustainability, diversification, Traditional Ecological Knowledge (TEK), reciprocity, resilience, } \\
\text { adaptability, reclamation, identity, knowledge, nature, harmony, balance, the natural way of life }\end{array}$ \\
\hline
\end{tabular}

Table 8

Excerpted responses to question(s) regarding use of term sustainability and/or "how would you define 'sustainability'”?

\begin{tabular}{|c|c|}
\hline Use/definition of "sustainability" & Participant \\
\hline $\begin{array}{l}\text { "A short definition for sustain is to keep intact, to maintain, and that is a } \\
\text { deliberate decision. It's a deliberate thing to sustain, because you understand } \\
\text { the system, you understand its nature, so you take action not to harm } \\
\text { irreparably that system." }\end{array}$ & $\begin{array}{l}\text { Antone Minthorn } \\
\text { (Cayuse) }\end{array}$ \\
\hline $\begin{array}{l}\text { "I would probably say it’s similar to 'nature.' I was asked how to define } \\
\text { 'nature,' and it’s every living thing on earth....So, I think sustainability goes } \\
\text { back to nature, and everything that's here.” }\end{array}$ & $\begin{array}{r}\text { Carol Craig } \\
\text { (Yakama) }\end{array}$ \\
\hline $\begin{array}{l}\text { "Sustainability is a very high level abstraction word. When I think of } \\
\text { sustainability, I have to break it down a bit and say what does that mean for } \\
\text { indigenous people in this struggle? What does it mean for the land, the } \\
\text { struggle to restore the land? Words that come to mind are resilience, the } \\
\text { capacity to adapt to change, reclamation of our voice and vision, identity, } \\
\text { knowledge, places, names of places, land, harmony." }\end{array}$ & $\begin{array}{l}\text { Dennis Martinez } \\
\text { (O'odham / } \\
\text { Anglo / } \\
\text { Chicano) }\end{array}$ \\
\hline
\end{tabular}




\begin{tabular}{|c|c|}
\hline Use/definition of "sustainability" & Participant \\
\hline "As we (Haida) see it, it is living from the land without spoiling it." & Guujaaw (Haida) \\
\hline $\begin{array}{l}\text { "Sustainability on one level means to be able to maintain and sustain the } \\
\text { fullness of health that needs to be there for us to thrive, and for everything } \\
\text { else to thrive." }\end{array}$ & $\begin{array}{l}\text { Jeannette } \\
\text { Armstrong } \\
\text { (Syilx) }\end{array}$ \\
\hline $\begin{array}{l}\text { "Home...Sustainability to me is keeping in touch with Mother Nature, valuing } \\
\text { a resource, conservation, preservation, the beauty of it all...Realizing that } \\
\text { everything that mother earth provides is how you eat. There is a big chain. } \\
\text { Everything serves a purpose. You take away one thing and it hurts } \\
\text { everything else.” }\end{array}$ & $\begin{array}{l}\text { Joeneal Hicks } \\
\text { (Ahtna) }\end{array}$ \\
\hline $\begin{array}{l}\text { "I don't know if I so much use "sustainability," because what I see is more } \\
\text { taking care of things, and protecting it, what we have, and trying to make sure } \\
\text { we will have that in the future." }\end{array}$ & $\begin{array}{l}\text { Kathryn Martin } \\
\text { (Ahtna) }\end{array}$ \\
\hline $\begin{array}{l}\text { "My way of thinking is that we have something for today, and something for } \\
\text { the future too. That's how I define it. Something that we have today that we } \\
\text { are going to need tomorrow too." }\end{array}$ & $\begin{array}{l}\text { Nicholas Jackson } \\
\text { (Ahtna) }\end{array}$ \\
\hline $\begin{array}{l}\text { "I don't have one really fantastic definition... what comes to mind is our } \\
\text { individual and our collective consciousness, that our resources and our land } \\
\text { and our time here on earth don't necessarily belong to us, it belongs to our } \\
\text { children and our children's children and it's really that consciousness and that } \\
\text { thought of being stewards of whatever that resource is. Be it our culture, be it } \\
\text { our values, be it our environment, be it the land, those are all resources that } \\
\text { we have and how do we protect it and sustain it for our children's children.” }\end{array}$ & $\begin{array}{l}\text { Nichole Maher } \\
\text { (Tlingit) }\end{array}$ \\
\hline $\begin{array}{l}\text { "The part that I think is so interesting about the word "sustain," is sustenance. } \\
\text { The roots of the word have to do with giving life and livability." }\end{array}$ & $\begin{array}{l}\text { Roberta Conner } \\
\text { (Cayuse) }\end{array}$ \\
\hline $\begin{array}{l}\text { "I guess I would define it as...it's got to start back at our culture. I mean, } \\
\text { that's the foundation for our sustainability is our culture, our identity, who we } \\
\text { are. You don't have that, you don't have people that have ownership in who } \\
\text { the tribe is." }\end{array}$ & $\begin{array}{l}\text { Shawn Yanity } \\
\text { (Stillaguamish) }\end{array}$ \\
\hline $\begin{array}{l}\text { "Sustainability is all about the links between your traditions, your culture and } \\
\text { the norms that are being imposed upon you by an outside society. The links } \\
\text { that I am talking about have to do with the ability of any particular group of } \\
\text { people...to absorb what needs to be absorbed from the world at large, to } \\
\text { protect and preserve what it has to have in order to sustain itself." }\end{array}$ & $\begin{array}{l}\text { Wilson Justin } \\
\text { (Althsetnay) }\end{array}$ \\
\hline
\end{tabular}

In our worldview, everything is profoundly connected. ${ }^{2,8,12,13}$ Sustainability is a term that should be used with awareness of the whole and our connection there within. When used with a consciousness disconnected from our profound connection with all of creation, then "sustainability" is a disconnected term that will fail to take the whole into consideration. Whatever decisions we make from such a place will have domino effects, merely displacing problems at best. ${ }^{13}$ We must embody our awareness of the intimate and profound connection among all things. We posses an inherent intelligence, and if we operate with our minds and our hearts connected, then we will move in greater harmony with creation.

We believe that sustainability is about living with a consciousness that our time here on Earth, and all the gifts here are not ours; they are borrowed from our children and our children's children. As such, it is our responsibility to be stewards of the Earth 
during our time here to ensure that future generations are able to experience those same gifts during their time here. ${ }^{2,4,7,9,10,11,12,13}$ Doing so calls for making a deliberate decision that we will sustain these gifts. "2,6 "We need to look at the big picture and say once and for all that we want to protect this creation for our future generations." 2

Even if we commit to this decision, given the interconnectedness of our world, we recognize that for our communities to be sustainable, sustainability must be achieved globally. ${ }^{5,10,12,13 ~ " M y ~ p e o p l e ~ n o w ~ i n c l u d e s ~ e v e r y ~ p e r s o n . . . ~ b e c a u s e ~ y o u ~ a r e ~ a l l ~ c o n n e c t e d ~}$ to what happens in my home, and in my land, and what happens to my children, and my grandchildren." 12 We accept our responsibility to tend to our respective places in the world, and to share our knowledge and understanding with others who are willing to accept the same responsibility. ${ }^{9,12,13}$ 


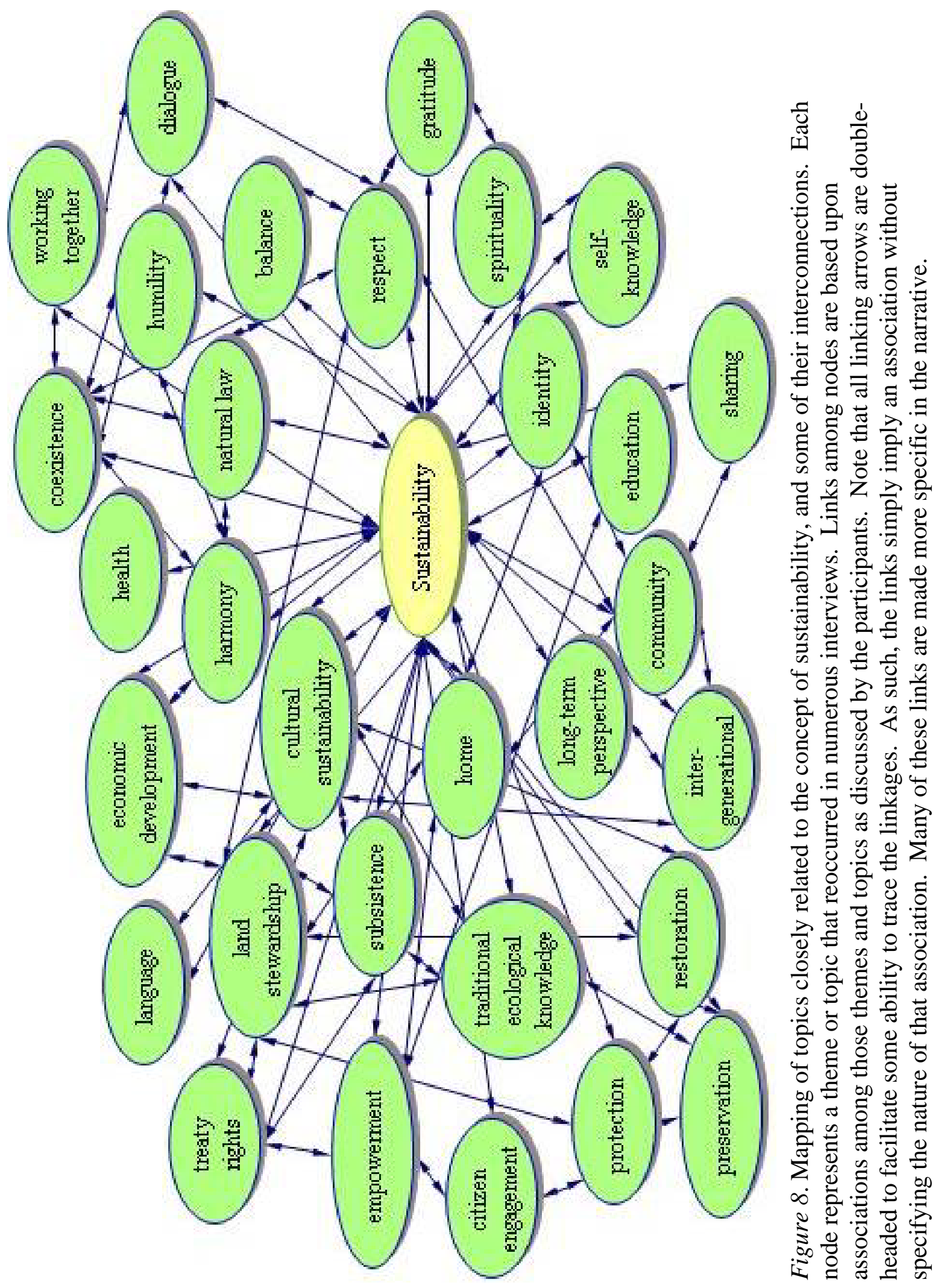


Taking Care

At its most basic level, sustainability is about survival: we have to have water and air and we have to have some form of sustenance. ${ }^{8,9,10,11}$ The shared roots of the words sustainability and sustenance, "have to do with giving life and livability." ${ }^{9}$ Had it not been for all our relatives, including the deer, the elk, the moose, the berries, the roots, the salmon, and many others, we would not be here today. ${ }^{6,8,10,11}$ Especially during challenging times, we must take care of and protect these things that are our life source,

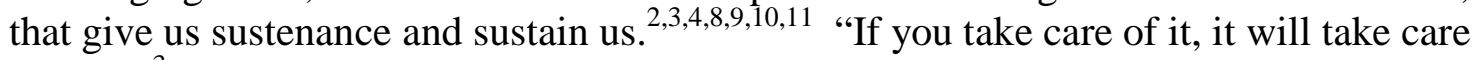
of you." 3

Taking care is about living in accordance with natural law. All our plant and animal relatives live according to natural law and require certain cyclical processes to sustain themselves. We must respect these processes so that we do not destroy the foods. $2,3,6,9,10,13$ "If you violate that law, to where you break that cycle, then you pay the consequence and whatever that may be." ${ }^{6}$ In other words, "there is an order to things, there is a balance to things, and that balance is sustaining. If things are out of balance, we have difficult times." 9 Decisions such as clear-cutting a forest to maximize immediate economic gain compromise the balance, violate natural law and create difficult times. ${ }^{9,11,13}$

Considering natural law, the true meaning of sustainability is not theoretical, it is practical. $^{7,9,12}$ There are clear implications for what to do and what not to do. Our traditional laws, ceremonies, and teachings are all about giving guidance to appropriate ways of living and interacting with the landscape and as a community to ensure that our behaviors are not a danger to ourselves, all else around us, and the generations to come. 5,7,9,12,13 Maintaining and sustaining the fullness of health needed for us and everything else to thrive is our responsibility, and that is what our intelligence, creativity and the gift of being human is about. "If we cannot measure up to that, and we cannot live up to that, we're not needed here, and we won't be here.” ${ }^{12}$ Alternatively, we can accept responsibility to live in balance with natural law by looking after the land that provides for our needs and by not abusing the gifts of the Creator. $3,8,9,10,11,13$

\section{Economic Development}

Sustainability is about harmony between ecology and the economy that a community of people engages to meet their needs. ${ }^{6,9,10,11,12,13}$ Such harmony requires very intimate knowledge among the people about the local ecology. ${ }^{10,13}$ We possess greater knowledge than many give us credit for, and with empowerment to design our own economic systems, much can be done to provide for culture and economy and environment. ${ }^{6,9,11,13}$ That does not call for a hands-off approach: it is "a matter of how you do it and where the limits are drawn. For instance, taking all of the fish from a river will give you a pocket of money, but taking only a portion of those fish over many decades provides far more over time, potentially in perpetuity." ${ }^{11}$ Such an approach requires people to be "able to maintain their need level consistent with their ability to meet that need." ${ }^{5}$ This calls for personal humility and resisting temptations to accumulate more personal wealth than is necessary., ${ }^{2,9,11}$ "There should be enough here for everybody to live, eat well and be comfortable, the trouble is that too many people want more than enough." ${ }^{11}$ A guiding rule is to always take only what you need, and make good use of that which you take. ${ }^{3,9}$ Perhaps such an orientation is the antidote to 
concerns of overpopulation. This has not been tested, and we believe it is a possibility worth testing. ${ }^{11,12}$

Given the difficulties of today's circumstances, there are some aspects of our thinking on economic development that differ among us. Some of us believe that given the context of the larger socio-economic system within which we are embedded, we cannot let progress pass us by, and we have to keep up with the times. ${ }^{6,8}$ Conversely, some of us call for a completely radical realignment in the way we think and how the socio-economic systems operate. ${ }^{5,10,11,12,13}$ We can agree that an economy is necessary to provide for our needs, but questions remain as to how to achieve an economy that aligns with our cultural values and restores the environment. Answers to these questions are likely to vary with each community's degree of empowerment and the amount of viable opportunities available.

\section{A Human Focus}

While appropriate economic development is important, we believe that the conversation around sustainability should be more focused on us as human, as opposed to focused on commerce and how to sustain levels of resource utilization for human purposes. ${ }^{5,12}$ A human focus calls for attention to the knowledge we have of ourselves and our environment, with focus on how to make a good life in coexistence with one another and other species. 5,7,8,11,12,13 A human focus means a focus on how our families and communities function, and how to equitably distribute our resources. ${ }^{5,7,8,12,13} \mathrm{~A}$ human focus in this sense forces reflection on some very important questions: What does it mean to be human? What does it mean to be indigenous? What does it mean to be a part of this planet and one of the life forms? What is our role and what knowledge and skills and values do we need to cultivate to be responsible participants? How do we build community where we are all cared for and belong? How do we build community so that we function in balance and unity as a whole healthy organism? ${ }^{7,8,9,12,13}$

In reflecting on these questions, kincentricity may be a useful word to represent a key aspect of our thinking. Kincentricity means living with recognition that we are all related, not just with our biological family and other human relations, but also with plants and animals with which we depend upon and share the landscape..$^{2,8,9,10,11,12,13}$ If all people were to really believe, internalize, and act on the idea of kincentricity then "there will be more harmony between natural systems and human societies," ${ }^{13}$ because as members of a kinship system we are a part of an agreement to be responsible for all of our relatives. Honoring that responsibility will translate to respect, care and stewardship for the welfare of all our relations. 9,13 "If we consider that as being the paramount value we have, then everything we do, everything we think about and so on, and the ways we derive our economic livelihoods, we should make every effort to include our relatives in a respectful way, and do things that promote their welfare. That is coming from a spiritual place." 13

\section{Spirituality}

Spirituality is important and relevant in this conversation about sustainability. ${ }^{2,3,5,7,8,9,10,12,13}$ First, we acknowledge that each individual has their own experience and personal truth, and is entitled to pursue their own path. ${ }^{5}$ While words can never fully capture its full essence, we speak of spirituality in part as being centered and 
living from the heart, ${ }^{8}$ being connected, ${ }^{3}$ being whole, ${ }^{9}$ being respectful of all things. ${ }^{2,3}$ and fulfilling our responsibility as caretakers of the gifts from the Creator., ${ }^{8,12}$

Spirituality includes knowledge that while we are individuals and temporary in our bodies we are part of a larger living life force. Within that larger life force we have a role as humans to maintain the balance. Our essential nature calls upon us to be responsible for the care, protection, and love of the whole outside of our temporary selves. $^{12}$ In these terms, it is easy to see the link between spirituality and sustainability. In a sense, they are no different, for we must have the knowledge of ourselves as part of everything else, feel that understanding and give expression to it through our lives in balance with all things so that we may carry it forward as human beings from generation to generation. $^{12}$

Our ceremonies and traditional practices are vehicles for helping people experience that spiritual connection., ${ }^{5,13}$ They also teach people the ways to live out their responsibilities, and help put people in a positive place to do good work in their lives. ${ }^{13}$ Maintaining and restoring these aspects of our culture is critical to overall sustainability.

\section{Cultural Sustainability}

Much of our focus is on cultural sustainability, and "really focusing on the gifts and the strengths and the values and the beliefs that we have and carrying that forward." 7 Because culture and the environment are so inter-connected, we must work towards achieving both. Our principles and values promote conservation and environmental consciousness, and, we need healthy, abundant places to practice our culture. So again, both cultural and environmental sustainability are really essential and support one another. $2,7,12,13$

Cultural sustainability means continuously passing along the "the gifts of our values, the gifts of our beliefs, the gifts of our principles to our children, to our descendants, and a series of tools as well, about how to live." 7 It also means giving our children a strong sense of who they are, that they are wanted and needed and truly belong in our communities, and that they play an important role as our leaders of tomorrow. $4,7,8,10$ Some primary values and principles of our cultures that we believe are of utmost importance to pass onto our children and instill in them as our future leaders include: respect, reciprocity, and humility. "A value has value because it's multigenerational. It stays in place," 5 and these are a few values we see as worth sustaining as they are important to sustainability.

Respect. "Respect is one of the core operative principles of law amongst our people," ${ }^{11}$ and it is a central value and practice needed for sustainability. ${ }^{2,3,6,7,9,13}$ Respect for the land and all life forms is shown through taking only what you need, making good use of what you take without wasting, and being appreciative of all the gifts. Respect for others is shown by sharing, ${ }^{2,3}$ not being judgmental, having love and understanding in your heart, ${ }^{8,12}$ being very thoughtful in what you do and say, ${ }^{7}$ and honoring ways of life that differ from yours. ${ }^{2,4,5}$ All this comes back to having respect for yourself, as you are dependent on others and the gifts of the Creator. ${ }^{4}$

Reciprocity. Closely related to respect, reciprocity is about maintaining the balance of coexisting and being in relationship with other species and other people. ${ }^{7,9,13}$ Reciprocity means being in true partnership in the sharing of space and resources. ${ }^{7}$ You are mutually dependent on the other, so you never take all of anything and you give 
something back whenever you take anything. This maintains balance and ensures something is left for others and yourself in future years."13

Humility. "People aren't the most important thing in the universe. We're not more important than other things." ${ }^{9}$ We are a part of this world, we are not in charge of it, and should not assume we know which parts of the whole are more important. Taking more than we need lacks humility and runs the risk of upsetting the balance. ${ }^{9}$ We have much to be grateful for, and showing our appreciation is part of having humility. ${ }^{9}$ Also, others may hold knowledge we do not, so we must be humble to be open to learn from what they know. 5,12

In addition to promoting these values, our diverse cultural traditions serve to keep us healthy, physically, emotionally, and spiritually. Our cultures provide the space through ceremonies, dance, music and song, language, and art to help our people maintain free movement and flow of the life force within, keeping us individually and collectively in harmony with all of creation. ${ }^{13}$

\section{Home}

An idea that ties together much of what has been mentioned thus far is that of home. Home is the place that sustains us and has sustained our ancestors for thousands of years. ${ }^{6,9,11,12}$ Home is where our cultures developed. ${ }^{2,6,10}$ Home is where our kinships formed; the place of all our relations. ${ }^{2,4,10,12,13}$ Home is a place we can call our own and be empowered to make the decision to sustain all our relations for the benefit of future generations. ${ }^{6,8,10}$ If you know you are home, and never intend to leave, then it is never in your long-term interests to harm the place you live. ${ }^{2,9,10}$

When you live in one place for many generations, an understanding and connection develops that is very deep, ${ }^{9,12,13}$ "as people know if they're third and fourth generation farmers or ranchers." 12 “The more intimate and familial our knowledge is of landscapes and species, then it's no longer an impersonal destruction that we're involved in. It's a personal reconstruction, a personal restoration." ${ }^{9}$ When you develop a deep connection with, and a deep knowledge of a landscape and everything that belongs there, then sustainability is the natural byproduct, it is the natural way to live. ${ }^{9,11}$ Now is a critical time for us to demonstrate our love for the places we call home. ${ }^{2,6,9,10,12,13}$

Extending from these thoughts about sustainability, we offer the following as an expression of our hopeful vision for the future of our communities that call Salmon Nation home. 


\section{Our Vision}

We understand that there is one world, and all things are connected. Each of us understands ourselves as existing as part of a larger whole, and inseparable from all that is. ${ }^{12}$ We know who we are as Native people, embracing our connection to our homelands and our cultural heritages. Our identity takes meaning and importance as defined by our sphere of responsibility., ${ }^{9,12}$ As members of a community, we are responsible for other people, the lives of young and old, human and non-human. We are responsible for the landscape that sustains us, and we give more than we take, passing along these gifts to future generations. ${ }^{2,4,7,8,9,10,11,12,13}$ We are respectful and thankful for all these gifts that make us who we are. ${ }^{2,3,8}$ Understanding ourselves within this larger context, we have pride and confidence in ourselves, knowing we are smart, strong, eloquent and beautiful people. ${ }^{2,6,10}$

We revere the beauty and significance of all life, honoring the rights of all species and peoples to exist. We accept our responsibility as caretakers and stewards. Due to our commitment, worldwide there is no animal, or bird, or fish, or plant that is on the endangered species list. Wealth and power are distributed equitably, and there are no peoples who are in danger, or at risk of disappearing. ${ }^{7,12,13}$

Within our communities we have found the balance between modern innovations and preserving our cultural traditions. ${ }^{4,12}$ Our children may play video games but they also play in the trees. ${ }^{4}$ New ways and technologies are filtered through the wisdom of tradition, ensuring we continue to adapt and thrive while not recklessly experimenting. ${ }^{12}$

We have also achieved a balance in the feminine and masculine forces inherent in all of us and all communities. ${ }^{12}$ We are strong and we are nurturing. We speak out and we listen. We attend to the tasks that need to be done and we are sensitive to the impacts our actions have on others. We utilize our minds and we live from the heart. By balancing innovation and tradition and the masculine and feminine, our community is able to function as a whole, healthy organism. ${ }^{12}$

All renewable resources are passionately protected, knowing the livelihoods of future generations depend on them. All the normal things that our past generations were able to do continue to be enjoyed. We walk among and marvel at the big trees. We welcome the salmon back as they run thick through the many streams. Sheep once again blanket Sheep Mountain. We hear the song of the meadowlark. The moose and elk that sacrifice their lives for us are rich in fat. We concede passage to the caribou when they block the road. We pick the plentiful fruits and berries, and we pull clams and pick seaweed at the beach. We breathe fresh, crisp air, and drink fresh, clean water. $2,3,4,6,9,10,11$

Thanks largely to the abundance of the land and our intimate relationship with it, we are healthy and happy people, ${ }^{2,3,4,6,7,8,10}$ living long lives, diabetes free ${ }^{6,8,9}$ and drug free. ${ }^{4,6,8,9,13}$ Our people choose not to use drugs and alcohol because they know who they are and have direction in their lives to better themselves and the community. ${ }^{4,6,8}$

Strong functioning family units are the backbone of our community enabling the development of the individual skills and values required for us to sustain our ways of life and the land. ${ }^{5,6,7,8,10}$ Mothers and fathers, aunts, uncles, grandparents, all play important roles in the development of our children. Our elders are valued and respected. They feel valued and work to pass down their knowledge and skills to younger generations. ${ }^{3,4,8,10,12,13}$ 
The processes by which knowledge and values are transferred inter-generationally are seamlessly woven into everyday life. ${ }^{5,12}$ Stories are regularly told ${ }^{13}$ and our children are involved in the on-going activities of conservation, restoration, and subsistence living. ${ }^{3,7,13}$ Our children learn through these stories and activities to be humble participants in life, to be appreciative of all of our gifts, to engage in reciprocity in all our relationships, and to show respect for all life forms. ${ }^{9,12,13}$

Respect is central to our lives. , $3,4,6,7,8,9,11,13$ We recognize the sacredness of taking another life to benefit our own. We respect the land, plants, fish and game by taking only what we need and not wasting any of what we take. We make beautiful and useful things from our plant and animal relatives to show our respect for their sacrifice. ${ }^{9,11}$ Living in this way helps us to live in balance and harmony with natural law.

We share with one another, especially with our elders. As individuals and as cultures we are known for giving more than we receive. ${ }^{3,4,7,9,11,13}$ Sharing brings our community together, and lifts the spirits of all. When together, humor is shared, filling the air with laughter, keeping our hearts light. ${ }^{10}$

We encourage our children to live their dreams and we strive to make those dreams attainable. Through formal and informal modes of education, we prepare our children with the skills they need to be successful and able to adapt wherever life takes them and whatever challenges are faced. ${ }^{5,6,7,8,10}$ Our children are well educated in both Western thought and our traditional knowledge. ${ }^{7,13}$ In the classroom, our children benefit from having good teachers who teach and live our knowledge and values. ${ }^{5,7,10}$ Our children continue with interest in their own education, many pursuing advanced degrees, and many returning home where rewarding opportunities await to serve and teach the next generation. ${ }^{4,5,7,8,10}$

We put forth leaders who are principled people, with their principles rooted in the values and practices of our cultures. ${ }^{11}$ Our leaders are strong, determined, disciplined, and knowledgeable yet humble, with the skills needed to improve the wellbeing of our communities. ${ }^{5,6,13}$ Our leaders are visionary and proactive towards addressing problems 5,6 with the moral courage to speak out against injustices, and the moral integrity to avoid pursuit of self-serving agendas. ${ }^{5}$ They give clarity to issues and circumstances, empowering others to make wise choices and take action., ${ }^{5,11}$ Our leaders are great listeners. They truly listen to others as well as listen to their own heart and "let spirit speak" to them ${ }^{8}$ as they serve the long-term interests of our people.

Our treaty rights and sovereignty are fully recognized, empowering us to make decisions, design our economies, and live in alignment with our values. ${ }^{6,10,11,13}$ Guided by our values, traditional ecological knowledge (TEK), and our inherent intelligence, our economies function to meet our needs in a manner that simultaneously promotes the abundance and diversity of the land. ${ }^{12,13}$ The historical patterns of relationships interconnecting our Native communities are restored, enabling the sharing and exchange of resources for cultural needs and enrichment. ${ }^{8,9,10}$

We are respected as indigenous people. People from within and beyond the region know of the diversity of our cultures. Visitors to our home respect our lands and our cultures. Educational opportunities are available to them to learn our stories and ways of living. They observe us acting with care and respect and they learn to act in these same ways. Visitors leave with greater appreciation and an understanding of who we are and of the shared responsibility to care for Mother Earth. ${ }^{2,3,4,9}$ 
We continuously build strong collaborative relationships with other organizations that understand and respect our cultures, and are willing to work in true partnership towards protecting the rich natural and cultural treasures of our

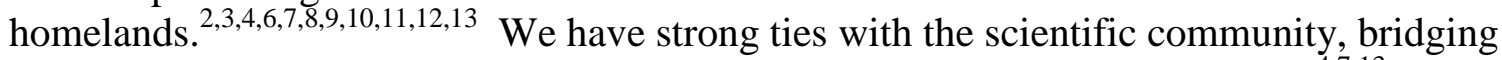
Western science and TEK in education and in addressing real world problems. ${ }^{4,7,13}$ Local, state and federal agencies understand our needs and work with us to find solutions that work for everyone today and in the long run. ${ }^{2,3,4,6,7,8,10}$

Together we continuously employ our creativity and intelligence as human beings to ensure that all aspects of creation move forward to be experienced and enjoyed by future generations. 


\section{How We Get There}

The process by which we pursue sustainability and our vision is as important as the goal, and the process must be harmonious for the outcome to be harmonious. It starts within ourselves: we must individually reconnect with the "real human being" and inherent intelligence inside ourselves before we are able to fully act from a place of wisdom and create harmony in our relations with the world. This calls for individual and collective reflection on the questions of who we are as human beings, and what our role is on this planet, and why we are where we are today with all these problems. This soul searching will help steer us in a wiser direction. From there we each need to take responsibility within all of our roles, as citizens and as consumers, as employees or as employers, as local or national or international leaders, as sons and daughters, and as mothers and fathers. The actions needed today must be engaged in all places at all levels of society. ${ }^{7,9,12,13}$

\section{Leadership}

"Somebody's got to step up and begin taking the leadership part in dealing with these problems. Otherwise, if you're laid back it's just going to continue. Somebody has to take responsibility." 6

We must walk the talk, because many learn not by being told what to do but by observing the actions of others. Thus, we must start with ourselves, and from there teach our families, other members within our communities and beyond to assume responsibility for the protection, care and restoration of our cultures and the land. ${ }^{4}$ To promote the active engagement of all, we need our leaders to speak up with clarity and courage to bring further awareness and understanding to the importance of the work that needs to be done. $^{2,13}$

We find hope in the promise of our youth to assume the leadership roles of tomorrow, ${ }^{2,7,13}$ and to carry a vision that crosses the boundaries and constraints we have created and perceive as adults. ${ }^{5,9}$ Still, much work needs to be done to prepare the youth and tribal members of today to be the leaders of tomorrow. ${ }^{6,8}$ In addition to providing quality education and developing problem solving skills, ${ }^{5,6,7}$ we must ensure that they are educated about the way tribal governance works, including relationships with the federal government, the state, and other tribes. ${ }^{6,8,10}$ Additionally, continually practicing our culture is important for instilling those values into our leaders of tomorrow. ${ }^{7,8,11,12}$

\section{Community and cultural restoration}

“Our cultures have so many values and have a lot of principles and a lot of world views mounted to them that really embody what sustainability is. But I think that those principles, those values, those ethics really come from a place of our culture and our heritage and so I just think it's so critical to build that heritage and to pass that on and to sustain those values and beliefs because if we can do that then we can really ensure that those 
same values that we're so proud about that protect our environment, that strengthen our communities, will continue forward." 7

We must mend the social fabric and dynamic for today's environmental threats to be resolved. Without intact families and communities, and transparent political processes with leaders acting on behalf of all people, harm will continue to be exerted on our peoples and the planet. ${ }^{5,12}$ Processes, such as naw'qinwixw of the Syilx people ${ }^{12}$ (see Appendix $M$ ), need to be engaged with great regularity to bring diverse voices and perspectives together so that our problems are addressed in a manner that works for the interests of all. Again, the process by which we pursue sustainability is at least equally important as the goal. ${ }^{12}$

Within our cultures are harmonious practices and processes that we must continually engage, especially with inclusion of our elders and our children. We should continue to tell our stories, ${ }^{3,4,9,10,11,12,13}$ to engage in art, song and dance, ${ }^{11,13}$ to speak our Native languages, $3,4,5,8,9,10,12,13$ to hunt, fish, gather and share our traditional foods, ${ }^{2,4,3,6,8,9,10,11,12,13}$ to actively steward the land, ${ }^{3,4,8,10,11,12,13}$ and to conduct our ceremonies $5,8,10,12,13$ for through these activities we achieve the transfer of knowledge, values, and life experience from generation to generation, and we reaffirm our connection with all things. $5,8,9,11,12,13$

Expanding on the importance of some of these aspects of our cultures, programs are needed to facilitate the learning of our Native languages to keep them and the knowledge embedded within them alive. . $^{3,8,9,10,12,13}$ Sharing with others, especially elders, is one of the most uplifting things we can do that brings us closer together as community. 3,4,7,8,9,11,13 And, we must keep the ceremonies going. In bringing us together to pay respects to the gifts of life, our ceremonies reconnect us with our cultural identity, provide a needed spiritual touchstone, and remind us of our responsibilities as humans to continuously live in balance. ${ }^{8,10,12,13}$

\section{Ecological preservation and restoration}

“Any renewable resource should be protected. To me, I think our creator created it for us to live off of and we need to protect it. If we don't, we are going to lose everything that was there for us. We can't live off of money, that's for sure. We can't eat money, we eat fish.” 3

Preservation of those places that are still relatively intact is critical. ${ }^{2,3,4,7,13}$ In addition to maintaining the refuge and services for all the species that call these places home, we need to preserve these places for models of the restoration work that needs to occur elsewhere. ${ }^{13}$ In some cases we need to establish legal mechanisms for protection. ${ }^{2,3,9,11,13}$ Where possible we should purchase lands for conservation and restoration. ${ }^{10,13}$ And wherever applicable, we must ensure the enforcement of tribal treaty rights for the basic social justice of being able to access to hunt, fish and gather, as well as enable stewardship of those lands via traditional management techniques. ${ }^{6,8,10,13}$ Ultimately, what really matters is what is happening on the ground, so when all else fails, we should be prepared to engage in the direct action needed to protect the forests, 
wetlands, salmon creeks, and everywhere in our homelands that has helped meet our spiritual and physical needs. ${ }^{11,12,13}$

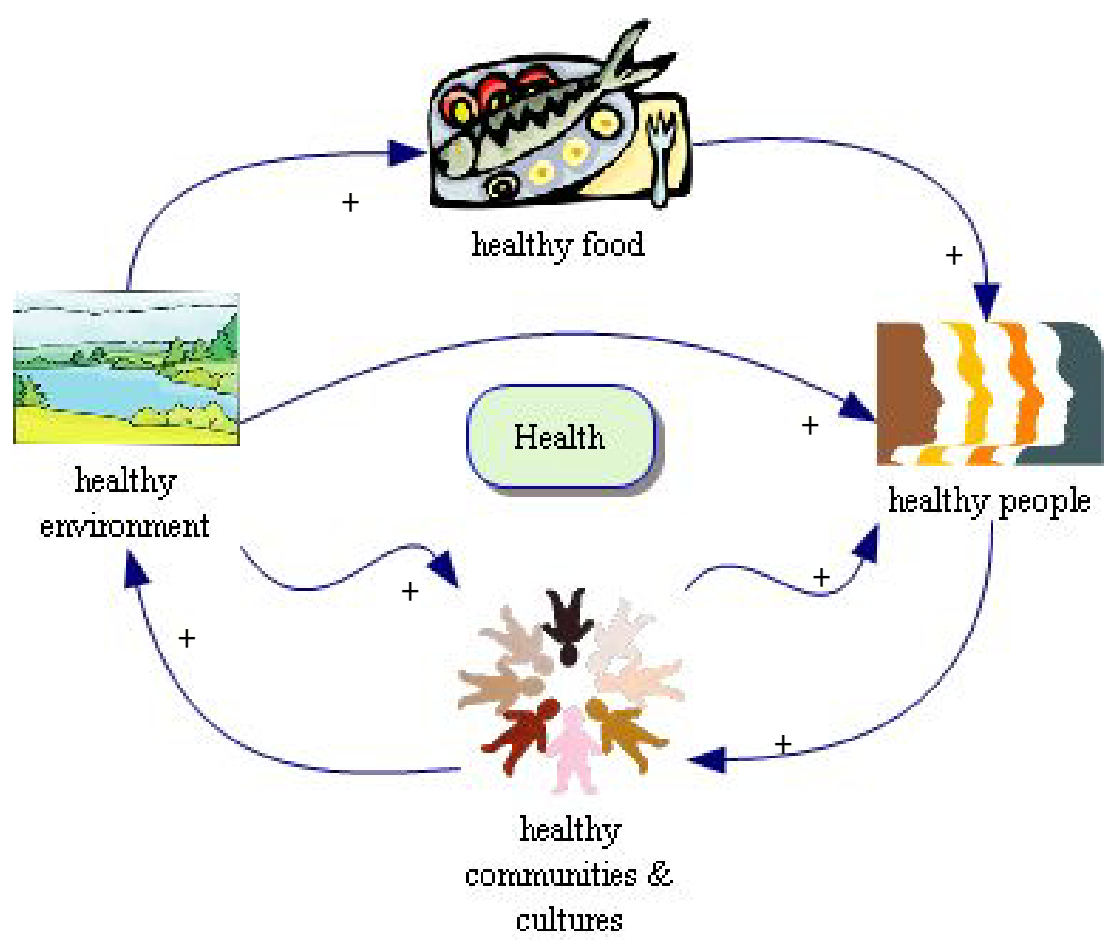

Figure 9. A view of health as resulting from interaction between healthy environments, healthy foods, healthy people, and healthy communities and cultures.

Build awareness, understanding and engagement

"There are some things we're still struggling with and fighting, but I think just the more education and communication that happens and understanding one another, that we'll be able to, I believe, continue on to have a better place for everybody.” 4

Education is one of the most important pieces in achieving a sustainable future for all. $2,3,4,5,6,8,9,10,11,12,13$ In the schools attended by our children, we need to work to integrate programs that teach our Native languages as well as our cultural knowledge and values. ${ }^{3,5,4,713}$ All children should receive a more accurate account of history, before and after the arrival of Columbus. Our heritage is now shared, and it's important for everyone to have a better understanding of North American history to understand the realities of today, and make right the injustices that have occurred. ${ }^{6,7,8,9,10,12,13}$ All children should also receive a better education about the way ecosystems function, the habitat needs of fish and game, and the importance of conservation. ${ }^{2,3,7,9,12}$ This calls for more time beyond the indoor classroom into the multi-sensory classroom of the out-ofdoors with real hands-on learning experiences. ${ }^{7,9,12}$ Involving our elders, inside and outside of the classroom, can really contribute to the learning experience children have from Native languages, to Native culture, to history, to traditional ecological knowledge (TEK). ${ }^{7,8,10,12,13}$ 
Formal education is an obvious place to start, but in every aspect of our lives we have opportunities to communicate, share our knowledge and demonstrate through our actions. ${ }^{4,5}$ We need to continuously work to increase people's awareness of the consequences of their decisions and actions. ${ }^{2,3,4,11,12}$ For example, almost all projects require investment money, so we need to do the work to inform investors of the kinds of impacts their money has on our communities. ${ }^{11}$ For issues involving the broader public, like recycling and proper waste management, public information campaigns, including commercials and advertisements, can help to promote awareness of these issues and cultivate environmentally minded attitudes. ${ }^{2}$ Also, everyone needs to take personal responsibility to be lifelong learners and be educated on important issues. ${ }^{6,7}$

As Native peoples, we need to be active in educating the general public and political leaders about our needs and perspectives. ${ }^{2,4,7,8,12,13}$ Part of this is making sure our voice is heard by being organized and participating in the democratic process. $2,7,13$ Knowledge and talk of what should be done will be just talk without the political will to get it done. ${ }^{7}$ As politically engaged people, we also need to be humble and educate ourselves about the needs and perspectives of others. With greater understanding of each other, there is a better chance that we can live and work together in greater harmony. $4,8,11,12$

Greater awareness and understanding of the problems and the needs of others will empower people to take action. ${ }^{2,7,11,12,13}$ We need all people to stand up with courage, confidence and hope, to find their voice and to commit their lives to finding solutions. ${ }^{2,3,6,13}$ The problems and the gifts of today are mutually inherited, so it is the responsibility of all people to become involved in the cause for a better tomorrow. ${ }^{7,9,12,13}$

\section{Work together}

"The loss of knowledge and the environmental crisis, the need to bring science and TEK together for the mutual benefit to all of humanity: it is no longer a color issue, it's not an ethnic issue, it is a humanitarian issue at this time." 13

We get there together. ${ }^{2-13}$ We need the perspectives and knowledge and visions of many to effectively deal with the crises we face. We must build true partnerships that are entered with humility and mutual respect such that we acknowledge the validity of alternative ways of seeing things. ${ }^{4,7,9,12,13}$ Whether it is within our communities, with neighboring communities and local governments, with representatives of state and federal governments, or with non-governmental organizations (NGOs) and big environmental organizations, the process of building relationships takes time and mutual effort to understand the perspectives, needs and interests of one another. ${ }^{2,3,4,7,8,10,13}$

Dialogue is a powerful tool for building healthy, united communities and building effective working relationships across communities. ${ }^{7,9,11,12,13}$ In particular, we desire a stronger dialogue with the environmental community. Indigenous interests of maintaining intimate relationships with the land are often challenged by efforts to set aside wilderness preserves to the exclusion of people despite long histories of human inhabitation. We hope to move beyond this conflict of interest with much of the environmental movement and achieve a more effective collaboration around our shared 
interest of protecting the planet. ${ }^{7,10,12,13}$ There is opportunity here to more effectively demonstrate the truth that people can live harmoniously with ecosystems and actually promote ecological health.

Integrating traditional knowledge systems with Western science is one of the most important and promising areas for effective collaboration. Much of the challenge lies in gaining further credibility in the eyes of scientists regarding the value that traditional ecological knowledge (TEK) offers, including long-term observations of place, proven models of sustainable agriculture, subsistence and forestry, and supportive value systems and cultural practices of those models. ${ }^{4,7,9,10,12,13}$ In addition to enhanced understanding of ecosystem health and functioning, the TEK/science partnership should intensely inquire into figuring out how to adapt traditional ways of knowing to modern times. ${ }^{7,12,13}$ Because much has been created in recent history without regard for our longterm interests, we need to engage processes or programs to evaluate modern achievements and innovations to determine what is actually useful. Wise evaluation calls for critical reflection through an integrated lens of traditional knowledge systems and science.

To achieve this integration, we must bring TEK practitioners and scientists together at conferences, convene special forums, and connect around on-the-ground problems $^{13}$. As this relationship develops, efforts should focus on integrating traditional ways of knowing into the formal educational curriculum so that the next generation of scientists inherently bridges the two. We see the most immediate opportunities at the level of higher education, but believe that this should continue to be pursued at all levels, especially among schools with large Native student populations. ${ }^{2,3,4,7,12,13}$

\section{Redesign}

"How do you do more with less, and treat the resources; the trees, the wood as precious commodities, and really treat the fish, and flesh, that you are going to either eat or transfer to somebody else as the very precious life that it is?" 11

We need to design economies that work with the values of our cultures and the local ecology. In many ways, this calls for the need to come up with radically different ways of doing things. ${ }^{5,13}$ A major challenge is with the global economy, which needs to be redesigned and instilled with a conscience so that it does not continue to wreck havoc on the Earth. This calls for reversal of trade systems and institutions that benefit the wealthy at the expense of the poor and indigenous peoples, while establishing truly fair trade, prioritizing self-sufficient subsistence living, and generally moving towards communal and conservation based economies. ${ }^{6,7,11,13}$ In essence, we must design our own future, otherwise someone else will dictate it for us. ${ }^{5,6,7,11}$

\section{Live our role}

"We're a part of this land, and necessary part of it. The land needs us, and the planet loves us, and we don't know how to be a part of that anymore, in a real sense, in a physical sense. A coming back to that is something 
that we as humans have to figure out together. We're all a part of that, we're all in that together." 12

The call to action is to serve as leaders in preserving and restoring our cultures and ecology. We must build awareness and understanding to engage all citizens of the planet in working together and redesigning our future. While the challenges are

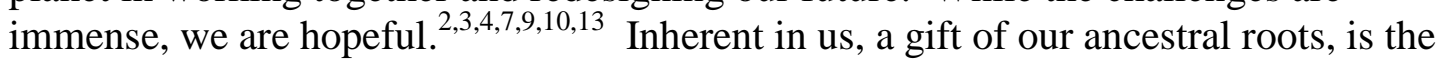
link with the larger living life force that connects us with all else. ${ }^{8,12}$ That gift is the source of our potential as humans to be active ${ }^{2-13}$ and careful, $2,3,4,6,8,9,10,12,13$ creative 6,8,9,12,13 and discerning, ${ }^{3,6,9,11,12}$ strong $^{6,8,10,12,13}$ and loving, ${ }^{4,8,12,13}$ intelligent ${ }^{2,6,9}$ and humble, ${ }^{2,4,5,9,11}$ respectful ${ }^{2,3,4,6,7,8,9,11,13}$ and grateful. ${ }^{2,8,9,10}$ Gifted with this potential, our purpose is to continuously maintain ourselves and all our relations in harmony and balance with natural law. The extent to which our cultures serve this purpose is the extent to which they are ultimately worthy of being passed along to future generations. $^{2,4,6,7,8,9,10,13}$

This is what we believe "sustainability” is about.

\section{Narrative Endnotes}

2 Hicks, J. (2007). Interview with Hall, D. E. Conducted June 19, 2007.

3 Jackson, N. (2007). Interview with Hall, D. E. Conducted June 20, 2007.

4 Martin, K. (2007). Interview with Hall, D. E. Conducted June 22, 2007.

5 Justin, W. (2007). Interview with Hall, D. E. Conducted June 22, 2007.

6 Minthorn, A. (2007). Interview with Hall, D. E. Conducted August 7, 2007.

7 Maher, N. (2007). Interview with Hall, D. E. Conducted August 17, 2007.

${ }^{8}$ Yanity, S. (2007). Interview with Hall, D. E. Conducted September 20, 2007.

9 Conner, R. (2007). Interview with Hall, D. E. Conducted September 25, 2007.

10 Craig, C. (2007). Interview with Hall, D. E. Conducted September 28, 2007.

11 Guujaaw (2007). Interview with Hall, D. E. Conducted October 20, 2007.

12 Armstrong, J. (2007). Interview with Hall, D. E. Conducted October 21, 2007.

13 Martinez, D. (2008). Interview with Hall, D. E. Conducted January 3, 2008. 


\section{CHAPTER VII}

\section{DICSUSSION}

This study inquired into the mental models of Native leaders of Salmon Nation on the subject of sustainability. Engaged in the tradition of social action research, the study sought to contribute to the dialogue on sustainability. The results represented in narrative form articulate a set of thoughts that represent a rich and complex understanding of the human experience as embedded and interconnected with clear implications for appropriate modes of behavior. The results were composed to stand on their own and serve as the primary source of input to the sustainability dialogue. Many topics have been invoked with relevance to all aspects of human life (and most academic disciplines) and are worthy of further discussion. In this chapter, I focus discussion on several of the major themes from the interviews, including identity, interconnectedness, active stewardship, restoring relationships with the land, restoring community, the bridging of TEK and Western science, and culture. Each major theme opens with a direct quote from one of the participants as a meaningful descriptor of the section, and to help maintain interview content as primary in informing the discussion. Thoughts about future research directions are shared throughout the coverage of the major themes. First, I acknowledge the primary limitations of the project, helping to appropriately qualify the results and the discussion that follows.

\section{Limitations}

The project covered a large geographic region and engaged leaders from a variety of communities and in varied roles. Subsequently, many of the specific thoughts and points did not filtrate into the narrative summary or following discussion. While much attention was invested to avoid the issue, ideas and specific quotes that were included run some risk of being misinterpreted without benefit of the full context they were shared. Additionally, because of the more generalized themes, the specific circumstances and needs of any one community may not be sufficiently addressed. Identifying commonalities across this broad region predisposes the project to lack detail on specifics of any one set of circumstances.

While the sample size of thirteen is a healthy number of participants given the employed methodology, it is certain to raise doubts about generalizability. As stated earlier, generalizability was never a goal. Rather, the goal was to purposively sample people knowledgeable about the topic of study (see Morse, 1991; Patton, 1990, 2002). The decision to sample primarily from the group of leaders affiliated with the Buffett Awards ensured participants held expertise in knowledge, as well as respect within Native communities, but it also generated the potential for screening out other perspectives discordant with the values guiding the Buffett Award process. Subsequently, the full range of perspectives on sustainability among knowledgeable indigenous leaders of the region may not be represented in the project. Rather than claim generalizability, it is appropriate to leave the determination of relevance and applicability of the project's content to each individual reader and her or his unique perspective and situational context. This idea is consistent with the notion of transferability as discussed by Lincoln and Guba (1985). 
The research was more successful in identifying convergent areas of thought than it was in identifying divergent areas of thought. The exercise of composing the narrative summary revealed that while there were certainly differences in perspectives and emphasis, these differences were more complimentary than they were contradictory. One clear exception to this was on the subject of economic development, as acknowledged in the narrative. Certainly, more time and effort could have been invested in analyzing differences, but the decision was made to focus on integrating the shared perspectives into one coherent representation. This decision was made largely due to the extensive range of topics covered across all interviews, and the constraints within any one interview to cover the full range of topics. Additionally, identifying aspects of shared thinking and language around the subject of sustainability was a primary goal from the outset of the project (see Appendix C). The decision to compose a unified narrative to emphasize shared dimensions of perspectives was criticized by one participant, who ultimately chose to have his name removed from association with the narrative content (Appendix J offers more on his perspective). It is acknowledged that the collective narrative statement does come at the expense of obscuring the diversity and uniqueness of each contributing perspective. Readers are once again encouraged to view the full interview content of each participant for greater depth and detail of their respective perspectives (see www.nativeperspectives.net). Readers are also reminded of the diversity across and within Native cultures (e.g., Manson \& Trimble, 1982).

Every aspect of this project has been constrained by the use of the English language. The linguistic relativity hypothesis proposes that embedded in the structure of a given language are characteristics of the worldviews of the cultures from which that language was developed, influencing perception and thought of its users. Strong versions of the hypothesis (e.g., Carroll, 1956) that language determines thought are largely rejected, but support is held for weaker versions that a particular language may be more or less effective in conveying and understanding certain concepts or ways of thinking (see Gentner \& Goldwin-Meadow, 2003). All interviews were conducted in English, a second language for a number of the participants. English, as a "Standard Average European” language, is characterized by linear relationships between subjects and object, with analysis rooted in objects in space, including references to past and future as "places.” By contrast, many Native languages in North America are more process oriented and cyclical (Haspelmath, 2001). Subsequently, the ability to convey certain ideas and relationships may be hindered, an issue raised by multiple participants as they attempted to convey their thinking. For example, "I think in pictures not in words. So I have to struggle to bridge that in order to communicate in the Western world" (L. Merculieff, personal communication, June 18, 2007). Consider that the translation first occurs in the mind of the participant, is spoken, reduced to the written form (losing details of tone and emphasis), summarily paraphrased by the researcher, then read and interpreted by the reader. Given the translations and transformations involved, preservation of precise meaning as intended by the interviewees can not be guaranteed. This limitation is not confined to this project (and is rarely acknowledged in other research), but it is a notable limitation given the purpose to unearth and represent mental models and meanings through heavy dependence on the English language. With this limitation and the others in mind, further discussion is now offered to reflect on a number of the major themes from the interviews beginning with identity. 
Identity

The idea of the word that we use to describe ourselves, Syilx people... if I were to translate that word [Syilx] for you, contained in that word is the foundational instruction, or paradigm, that expresses that idea of being so indigenous, and so a part of the natural word that our humanness is an expression of that natural world. (J. Armstrong, personal communication, October 21, 2007)

Our sense of self is a central force influencing the way we see, think, and behave (e.g., McCall \& Simmons 1978; Rosenburg, 1981; Turner, Hogg, Oakes, Reicher, \& Wetherell, 1987). Through my studies I have come to understand sustainability as fundamentally about a challenge towards self-preservation (see Chapter IV). The identities individuals, communities and whole societies hold of themselves, inform the meaning of sustainability in terms of what, how and why to sustain. Close identification with the greater whole of the natural world, motivating the desire to be caretakers and respectful participants, is a perspective clearly expressed in the interviews. As Yanity stated, "The salmon, the trout, the elk, the deer, that's part of our culture, and to have those disappear is another piece of our culture that is being taken away. We're responsible for those gifts that the creator had given us. That's why we take natural resources so seriously" (personal communication, September 20, 2007). Here we see that the very identity of the culture is defined in large measure by its relationships with these animals, necessitating the preservation of those species to ensure the preservation of the human culture.

The cultivation of an identity that extends beyond the individual is also seen in responses to the "I am" statements exercise (see Appendix G). These statements were shared in the visioning section of the interview script, and they speak to the sense of self that community members might hold in a sustainable future. Expressions of identity extended from individual-level attributes all the way to a universal identity (see Figure 10). Collectively, these expressions of self speak to an identity defined by relationships and responsibilities as subscribed by those relationships. For example, identification as an aunt or uncle conveys recognition of the responsibilities of one who plays a role in contributing to the development of a child within the extended family. Such identification was at the social level (e.g., "I am responsible for young lives and old lives.”) as well as the ecological level (e.g., “I am a consumer.”). The universal category of identity was established to represent the "I am" response of one participant who refused to engage in making categorized classifications of the self, recognizing that doing so set the stage for a separated, disconnection understanding of one's place in the universe. While such a response was not shared by others in the "I am" statements exercise, this expansive view of being connected with all that is in a spiritual and literal manner, was expressed elsewhere by numerous other participants. Between the "I am" statements and expressions of identity throughout the interviews, a clear link is established between the importance of self-concept and engagement in socially and ecologically responsible behaviors. 


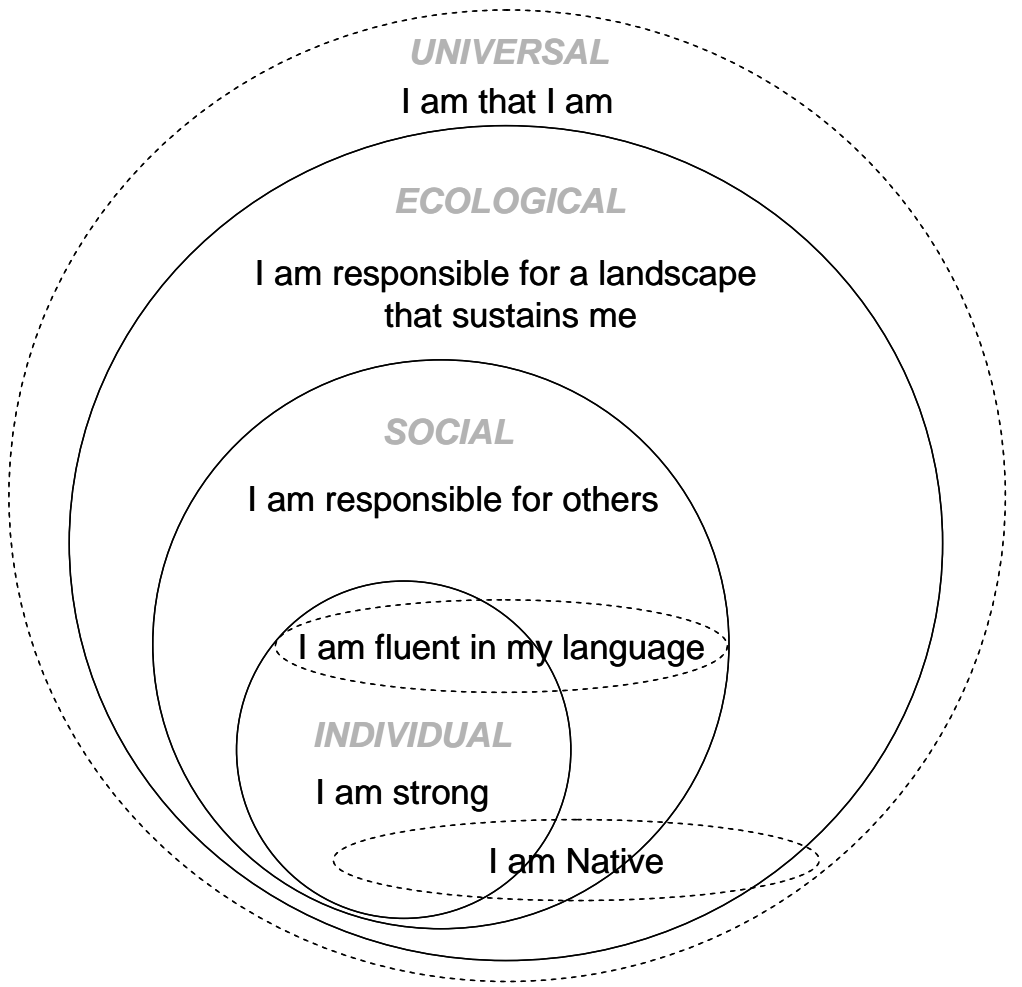

Figure 10. Representation of identity, or sense of self, based upon participant responses with example "I am" statements. See Appendix G for complete list of "I am" statements and definitions of each of the spheres.

Social identity theory (e.g., Turner et al., 1987) puts forward that beyond the individual-level, self is established through a process of self-categorization identifying with larger social circles or groups. Identification with these larger social circles establishes in-group membership, while simultaneously creating out-group categorizations. Individuals carry multiple categories of membership, internally organized in terms of their salience and importance in that individual's conception of self. In-group identifications, particularly those of greatest salience, then influence the way one feels, thinks and acts with preference displayed to that which benefits one's own group memberships, often at the expense of out-groups.

Among the participants, there is clearly strong in-group identification with their respective Native cultures. However, there is a stronger theme of cooperation than might be predicted by social identity theory, which "maintains that intergroup discrimination, differentiation, or bias is an automatic product of social identification" (Hogg \& William 2000, p. 90). This may be explained in part by some participants' identities extending beyond their primary indigenous social group to include all of humanity, and indeed all of life. For example, “my vision isn't just about my people because my people now includes every person: you in this room, and every person on this continent, because you are all connected to what happens in my home, and in my land, and what happens to my children, and my grandchildren” (J. Armstrong, personal communication, October 21, 2007). In this instance, in-group boundaries are understood to be too confining and restrictive to appropriately cope with the problems of a globalized world. Social identity 
theorists might suggest that cognitive-motivational processes engaged in a social context defined by globalization could give rise to an inclusive sense of self. However, the theory's emphasis on self-identification via relative social comparison constrains its explanatory power.

Another clear limitation of social identity theory as a framework for understanding these results is that the "social" identification for many of the participants was inclusive of plant and animal species, discussed in terms of kincentricity (see also Salmon, 2000). In addition to an interspecies identification, numerous expressions of identity were associated with specific geographic places. Together, a strong theme of identification with the broader ecological landscape is apparent throughout the interviews, and is consistent with the earlier discussion of commonly observed Native sense of self (e.g., Allen, 1979; Booth, 1996, 2003). Along with this identification comes a sense of responsibility and the role of serving as caretakers, as exemplified by Yanity's quote in the opening paragraph of this section, and discussed further below under the heading "Active Stewardship.”

Identity theory (see Stryker 1968, 1980) is no better at incorporating the observed ecological dimension of identity than social identity theory, but it does speak directly to the notion of role and responsibility. Like social identity theory, identity theory argues that a self-concept is developed through interactions in a social context. As such, self is seen as a social construct, based upon multifaceted roles defined for us by our social context (Stryker, 1980). These various roles then prescribe appropriate behaviors, and influence the dynamic of relating with others. In this manner, the societal structure gives shape and meaning to individual self. The participants of this project largely envision a future characterized by a societal structure that is both inclusive and nurturing of its members as well as instilling role identities that emphasize responsibility to others and the ecological landscape. For example, Maher shared that much of her thinking is focused on questions that include:

How do we ensure that our children have a strong sense of who they are and what their responsibilities are? How do we make sure that our children know that they are our future leaders? How do we make sure that they have the tools to be those leaders? How do we make sure that they belong to our community, that we show them we want them, that we need them and what do they need to do as a result of their important role in our society? (personal communication, August, 17, 2007)

While identity theory and social identity theory focus on the formation of a sense of self in a social context, failing to explicate on identification with the natural environment, Clayton (2003) puts forward a conceptual and operational definition of environmental identity (EID). EID is part of one's self-concept and defined by the extent to which a person holds a sense of connection with the nonhuman natural environment, his/her belief in the importance of the environment, and its importance as a part of who that person is (Clayton, 2003). As assessed by the EID scale, Clayton reports that EID serves as a strong predictor of environmentally conscientious behaviors, such as turning off lights when leaving a room. While a novel and valuable contribution, the conception and the EID scale are clearly developed with Euro-American and European populations in mind. Envisioning responses from the participating Native leaders of this project, 
items such as "My own interests usually seem to coincide with the position advocated by environmentalists" would generate highly mixed results due to the above noted inconsistencies between aspects of the environmental movement's agenda and that of Native interests. Nonetheless, Clayton (2003) adds evidence that identification with the environment serves as an important motivator for ecologically responsible behavior (the reader is encouraged to see Clayton and Opotow (2003) for more on the subject of identity and nature).

Research by Shultz (2001) maps out three broad categories of environmental concern: egoistic, altruistic and biospheric. The biospheric category, or concern for environmental issues based upon concern for plants and animals, demonstrated a strong relationship with pro-environmental behaviors. Schultz argues that this finding is best explained by the idea that those with greater concern for the biosphere have a cognitive representation of self that is more inclusive of nature. How might such a cognitive representation be promoted? Inspired by findings that adopting the point of view of others increases views of self to be more inclusive others (e.g., Davis, Conklin, Smith \& Luce, 1996), Schultz (2000) provides evidence that assuming the perspective of nature can expand boundaries of self concepts to be more inclusive of nature.

More research is warranted for exploring strategies to promote personal identification with nature. Additionally, more research is needed on the general subject of identification with the natural environment as well as on how such identifications are uniquely experienced by contemporary indigenous people. There is more to be learned about the extent to which such identities serve as impetus for engaging in ecologically responsible behaviors. Future research should also explore to what extent might existing structures and systems constrain individuals from enacting behaviors consistent with a sense of self intimately linked with the natural environment.

The thinking shared by the participating leaders reflected personal identification with social groupings from their immediate families, to all of humanity, and extending to non-human beings and the overall natural landscape. Without dictating the way others must live their lives, many also expressed the importance of cultivating an expansive view of self among the members of their communities and among all people. An understanding of self characterized by such ever-widening spheres of identification establishes a clear set of behavioral responsibilities, for individuals and social groups, for preserving the long-term integrity of the group requires preserving the web of relations that inherently define the group and the individual.

\section{Interconnectedness}

Everything around me has a purpose and they are intertwined to help me grow. Everything is dependent on another, like I am dependent on the fish and moose and so forth. If I want to keep it forthcoming in the years ahead, I have to preserve it and allow for production in the next year. I have to be thankful and appreciative for its existence and I can't take it for granted. (J. Hicks, personal communication, June 19, 2007)

The worldview of interconnectedness commonly expressed in the interviews and commonly cited as present among indigenous peoples (e.g., Cajete, 2000) is well 
supported by modern scientific understanding (e.g., Bateson, 1979; Capra, 2002; Laszlo, 1996). As Swimme and Berry (1992) suggest, "To be is to be related, for relationship is the essence of existence" (p. 77). Definitions of a "system" postulated by the young paradigm of systems science consistently include the idea of patterns of relationships (e.g., Lazlo, 1996; Lendaris, 1986; Rapaport, 1986). Relationship is central to the thinking represented in Open Systems Theory, which emphasizes the inherent closeness of the relationship between a given system and its supporting environment (Katz \& Kahn, 1978). So, the identity of a system is inherently tied to its relationships, both internal and external. Bateson (1979) went so far as to say that we are best defined not by our appearance and outward characteristics as they stand alone, but rather by our pattern of relationships with the world.

Remarkably, careful consideration of an entity's appearance and characteristics reveals embodiment of the world with which that entity has a history of relating. This understanding is captured by Croizat's (1964) metaphor, "space, time, and form: the biological synthesis.” Similarly, Bateson focuses on the presence of patterns that reveal relations within entities and between entities, or the patterns that connect. Bateson continues to discuss the significance of stories, not as something limited to a human communication strategy but as a fundamental feature shared by other living forms. Every aspect of our being is a story-we carry with us and represent all our experiences in this life and our genetic lineage in our physical form, behavioral patterns, and mental conceptions. In other words, we are contextually shaped, defined by the patterns of our relationships, and given sufficient regularity in interaction, ultimately embody those same patterns at the levels of our physical manifestation and psychic reality. In short, it is our pattern of relationships that defines us (Bateson, 1979).

Swimme and Berry (1992) provide an illustration of a bear born into a temperate forest. The bear's very form reflects the landscape and climate, from the thickness of its fur appropriately gifted to insulate from particular temperatures to the shape of its claw, which is patterned after the kinesthetics of the spawning salmon. Only through many generations of interaction and relation with a relatively consistent landscape and climate could the form known as bear come into existence. This illustration and the preceding thoughts serve to blur the distinction between these concepts of interconnectedness and identity.

The interrelatedness of these concepts may generate insight into a question posed by an interviewee and suggested to be worthy of serious further inquiry: impartial to the political and legal connotations associated with the term, what does it mean to be indigenous? Drawing upon this discussion of interconnectedness and identity, indigeneity may mean being the embodiment of a story that mirrors the broader story of a particular temporal and spatial context. Non-indigenous people have yet to engage in the reciprocal dynamics with geographic place for sufficient time to be so deeply connected that they are both defined by and define that place - although, given the extensive transformation of colonized lands, it could be argued that many places have come to be defined by non-indigenous people. Comparatively, consider the story of any given indigenous people. Generations of learning how to adapt and survive in their respective geographic region has given shape and form to their patterns of relating with the land, each other, and outsiders that defines the unique identity of each indigenous population. They can be understood as indigenous because the story of who they are requires 
knowing the context of time and place and the patterns of relationships that define them and their respective contexts.

Awareness of, sensitivity to, and reverence for this relatedness is a paramount characteristic of the thinking expressed across the interviewees. Such a perspective serves as an important mediator informing the manner in which one interacts with his/her environmental context. While the preceding section and much of this section emphasized the influence of context on human identity, the following section considers the reciprocal of the relationship; the human influence on environment.

\section{Active stewardship}

The land is suffering, all of our relatives are suffering, the plants and animals are suffering out there, because we are not taking care of them. We are not taking care of the land. (D. Martinez, personal communication, January 3, 2008)

The theme of active stewardship, or "taking care" as discussed in the narrative, stands out as one of the most compelling themes from the interviews. Active stewardship here refers to the intentional employment of interventions on the landscape to promote particular conditions and characteristics. A host of examples were cited by interviewees, from salmon enhancement strategies of the Tlingit, ${ }^{1}$ to the burning practices of the Walpole Reserve, the Three Fires Reserve of the Ottawa, Potawatomi, and Anishnabeq (Ojibway) that continue today. ${ }^{13}$ Speaking of the Okanagan region, Armstrong offered:

There is real clear evidence on some of our people who are doing research now that (with human seasonal migration) not having taken place in the last hundred years, our land is dying. Many of our plants and bird species are disappearing. Much of our area is still wilderness and undisturbed, but without the human intervention. So there's something about the human intervention, and the human role on land, and on environment that creates bounty, and that creates productivity, and that creates enhancement to the environment and in the biodiversity. (personal communication, October 21, 2007)

The case put forward clearly by Armstrong and the other examples cited make the argument that not only have many indigenous peoples inhabited places without deteriorating resources, they have actually enhanced the diversity and abundance through active stewardship. As such, in the Okanagan region and perhaps elsewhere, part of the ecological deterioration observed today may be due to a lack of human interaction. I understand that this invites renewed discussion about the "ecological Indian” as addressed in Chapter IV. As pointed out by Martinez:

Indigenous people are not noble savages nor are they ignoble savages; they are human like everyone else. They make mistakes like everyone else. They have made quite a few in the past, but the ones that have learned, adapted the knowledge, and the ways, they've survived. Those are the people we want to look to as models” (personal communication, January 3, 2008) 
Following Martinez's point, for me the question is not whether all Native peoples were good stewards of the land, rather, whether there are examples of such stewardship that demonstrate the potential for human beings to live in a symbiotic fashion that actually promotes species diversity and density, as well as overall ecological health. Several examples have been cited here, giving credibility to the idea while not necessarily resolving the larger ecological Indian debate.

Another example of restorative human intervention brought up by three different interviewees is the Umatilla Confederation's efforts to bring salmon back the Umatilla River (A. Minthorn, personal communication, August 7, 2007; R. Conner, personal communication, September, 25, 2007; D. Martinez, personal communication, January 3, 2008; see also, Phillips, Ory \& Talbot, 2000). It started with initiating a stakeholder dialogue to allow water to return to the river. Today, not only have salmon returned, but there has been a rebound in the populations of a whole host of other species as well, including black bear, cougar, osprey, golden eagles, and bald eagles. This example and the others speak to the potential for humans to be in symbiotic relationships with other species. We are capable of harm and we are capable of restoration. As Liz Woody shared, it is important for us to be aware of the full range of human choice from destructive to constructive, and be intentional in making the restorative choices (personal communication, April 17, 2007).

Due to extensive environmental destruction at the hands of humans, the modern environmental movement has largely adopted the strategy of establishing ecological reserves that exclude human habitation and minimize human interaction. This perspective is central to the justifications for forced relocations of indigenous peoples from those lands (e.g., Terborgh, 1999). Chapin (2004) challenges the approach, citing social injustice of the displacement of indigenous peoples. Further, this line of thought regarding the potential efficacy of active stewardship challenges the idea that such removal is necessary, or even ideal if the goal is preservation of the "natural" ecosystem. Indigenous people may play an important role in maintaining the "natural” ecology. Consider the Gwaii Haanas National Park Reserve, co-managed by the Haida Nation and the Parks Canada, and preserves the millennia-old subsistence rights of the Haida people within the park. In 2005 National Geographic Traveler surveyed 300 experts in sustainability tourism, destination quality, and park management and identified Gwaii Haanas as the best park in North America (Tourtellot, 2005).

Consider another example illustrating the potential for humans to serve in a restorative role while fulfilling human needs. The example comes from the Pacific Northwest where timber-dependent communities struggle in the wake of a dramatic dropoff in timber production (see Dumont, 1996). Meanwhile, the region is home to vast areas of overcrowded second growth that, relative to old-growth, are prone to more severe fires (Bury, 2004) and suboptimal for recreation (e.g., Englin \& Mendelsohn, 1991), habitat (e.g., Carey \& Johnson, 1995), and ecosystem services such as climate regulation and water purification (Creedy \& Wurzbacher, 2001). An alternative management strategy gaining credibility calls for selective harvesting of the weak and diseased trees, leaving the stronger trees behind. This results in less competition for sunlight and soil nutrients while also opening ground cover for younger saplings to take root and reestablish the age diversity of the forest and restoring the optimal ecosystem functions of the forest while providing sustainable economic returns (Donovan, 2000). 
This example in particular stimulates thought of the classic predator-prey relationship between wolves and elk. The elk obviously benefit the wolf as a vital source of sustenance, but the wolf also benefit the elk by keeping their population within the carrying capacity of the landscape and strengthening the genetic lineage of the heard by disproportionately killing off the weak and sick (see Boyd, 1994).

A series of examples have been illustrated, and the wolf-elk dynamic was cited to pose a question: is it possible that humans have potential to be a keystone species in that the well-being of other species and overall ecological functioning can benefit from our behavioral patterns of interaction? This question immediately forces confrontation with one of the most basic Western assumptions that we are somehow removed and separate from nature. Even most people who recognize humanity as a part of nature still see much of our creative and destructive acts as unnatural, fueling the belief that nature would be better off without us while justifying the position that conservation requires removal of humans from "wild" spaces. Contrast this belief with the theme found in this project's interviews that humans play an important role on the land historically, and most importantly, from this day forward play a critical role in the task of restoration. This is not to say that human behaviors are not to blame for the modern ecological crisis, but it is to challenge the notion that there is inherent conflict between humans pursuing need fulfillment and maintaining, even restoring, ecologic integrity. As noted, our current strategies of segregation result in social injustice, and do not necessarily result in optimal ecological outcomes. A mentality that our behaviors, even those involving economic incentive, bear the potential to actually restore and rejuvenate can open consideration of how to be symbiotic participants in the larger pattern of life on the planet. Clearly, realizing such potential would require intentionality, knowledge of place, awareness of the whole, and acting in balance with local contexts. Such requirements speak to the importance of restoring human relationships with the land.

\section{Restoring our relationship with the land}

The more intimate and familial our knowledge is of landscapes and species, then it's no longer an impersonal destruction that we're involved in. It's a personal reconstruction, a personal restoration. (R. Conner, personal communication, September, 25, 2007)

One of the stronger themes throughout the interviews, both explicitly spoken to and implicit among numerous accounts, is the importance of having an intimate relationship with the land. Historically, Native peoples lived lives intimately related with the land, but after generations of cultural assimilation, geographic displacement, and ecological deterioration, the degree of intimacy with the land among many Native peoples has declined. Kuhnlein and Receveur (1996) note the decline among indigenous peoples globally in traditional food systems and, subsequently, the knowledge needed to maintain those systems. While subsistence-based lifestyles are still more common among Native peoples, the opportunities for and practice of living off the land are dramatically reduced. Instead, the modern economic system serving human needs and wants largely disconnects people from the primary sources of their food, water, and basic consumer needs like clothing fiber and building materials. For example, the scale and complexities 
of the global food distribution system render the stories of the foods that grace supermarket shelves opaque to most consumers (Cook, Crang \& Thorpe, 1998). Subsequently, with food and other consumer choices, people are displaced in time and space from the consequences of their decisions. Additionally, the modern built environment obscures the salience of nature and minimizes cognitive awareness of one's inherent connection with the natural world (Schultz, 2002). Conner's quote opening this section provides a perspective that through intimacy and familiarity with nature people will be more inclined to disengage from destructive behaviors, and engage in restoration as they make the personal link with their own life.

Conner's perspective is supported by emerging theory, research and practice (e.g., Hay, 1998; Kals \& Maes, 2002; Orr, 1992; Pryor, Carpenter \& Townsend, 2005; Shultz, 2002; Trimble \& Nabhan, 1994; Vickers \& Mathews, 2002). Schultz (2002) discusses the idea of an individual's relationship with nature as being at the core of understanding the overall human-nature relations. He presents a model regarding inclusion with nature, which focuses on "the understanding that an individual has of her place in nature, the value that s/he places on nature, and his/her actions that impact the natural environment" (Shultz, 2002, p. 67). Three core dimensions characterize such inclusion with nature: connectedness, caring, and commitment. The idea of connectedness links with the above discussion about identity as it refers to the degree to which one includes nature as a part of his/her mental representation of self. Connectedness is suggested to causally influence caring, the extent to which one has feelings of closeness, affection and concern for nature. Schultz argues that such feelings only arise through sufficient knowledge of and experience with nature, the same as is required for such feelings to develop towards another person. An individual's pattern of connectedness with and care for nature is presented as leading to the behavioral dimension of commitment to protect nature. Commitment here refers to a willingness to invest personal energy, time and resources to protect nature and maintain a relationship with the natural world (Shultz, 2002).

Supporting Shultz's care-commitment link, emotional affinity for nature has been demonstrated as a significant predictor of environmentally conscientious behaviors (Kals, Schumacher \& Montada, 1999; Kals \& Maes, 2002). Kals and Maes (2002) discuss the construct of emotional affinity towards nature as being inclusive of other nuanced emotions, such as feeling good, free or safe in nature, or a "love of nature." Consistent with Shultz's argument about the link between connectedness and caring, the formation of emotional affinity towards nature is strongly influenced an individual's personal experiences with nature (Lyons \& Breakwell, 1994; Kals et al., 1999). While these construct definitions and findings are based upon research with non-Native populations, the general linkages likely hold across cultures. Bolstered by this research, it makes theoretical sense that direct interaction with nature promotes emotional connectivity with and caring for nature, and that such emotions would motivate pro-environmental behaviors. The obvious intervention strategy implied calls for promoting direct interaction with nature (Kals \& Maes, 2002), which coincides with the perspective of Conner and other participants. For example: "I think people just need some exposure and they need some opportunity to go there, to go the land and do something natural... anywhere that there's natural earth. As you get closer, you revere it, and maybe even join in the fight to save what's left of it” (Guujaaw, personal communication, October 20, 2007). 
In addition to promoting more ecologically sensitive behaviors, interaction and a personal relationship with nature may also be beneficial to the people's health and wellbeing (e.g., Hartig, Mang \& Evans, 1991; Maller, Townsend, Brown \& St Leger, 2002; Roszak, Gomes \& Kanner, 1995). Encouraging personal interaction with nature is an intervention strategy being actively employed in the emerging theory and therapeutic practice of ecopsychology (Glaser, 2008). Foundational to the theoretical perspective of ecopsychology is recognition that nature is where our minds and bodies evolved.

Subsequently, returning to spend time in the natural world can be comforting, inspiring, and restorative. Ecopsychology promotes personal reconnection with the natural environment, taking time away from the confining, over-stimulating and evolutionarily unfamiliar forum of the modern built environment (see Roszak, Gomes \& Kanner, 1995; Winter, 1996). Most participants shared a personal account of spending time outdoors as part of a healthy life, for example:

When I go out into the woods, or campout, or hunting, or whatever it may be, to me that's almost like my therapy. I get rejuvenated, reenergized, don't have the cell phone ringing every minute. Just kinda get away, and just get in touch with the land...Even spiritually, I feel renewed, and I can come back into the busyness of life again. (K. Martin, personal communication, June 22, 2007)

Similarly, adventure therapy programs utilize the outdoors as a forum for achieving healing (e.g., Beringer \& Martin, 2003; Pryor, Carpenter \& Townsend, 2005). These programs often are conducted in small-group formats, and seek to fully engage individuals in a holistic experience of mind, body and emotion. "This combination of aims constitutes the basis for a socio-ecological approach to health, where individual, community and environmental sustainability are integrated within a common approach" (Pryor et al., 2005, p.5). Complimentarily, outdoor education programs re-engage people with the outdoors to develop knowledge, skills and values regarding nature. Pryor et al. (2005) argue that in the context of a crisis in human and environmental health, outdoor education programs and adventure therapy programs are important aspects of a health promotion strategy. Adventure therapy programs are suggested to be best suited for targeted populations requiring a therapeutic intervention, while outdoor education is called upon as valuable as an entire population-wide intervention strategy. Particularly in terms of outdoor education, support for this argument emerges in a number of the interviews, exemplified by the following quote:

The classroom of the out-of-doors was the multicultural, multi-sensory, multigenerational classroom that Indian children had aboriginally, and it's a fabulous experience. Not just for one week in sixth grade, it needs to be life-long, but we can start K-12. I think absolutely, children out of doors, out of offices, out of classrooms, away from desks, best classroom ever. (R. Conner, personal communication, September 25, 2007)

I agree that outdoor education and adventure programs can play an important role in healing people and moving people to heal the planet. However, depending on how and where these programs are implemented, they may be displaced from the places people 
call home. Subsequently, the experiences people have through such programs may not transfer, or be viewed as relevant, to those places where they live and act out their everyday lives. Indeed, much of what has been discussed in this section so far as been about a general relationship with nature. Also needed is cultivation of an intimate and familiar relationship with the particular places people call home.

A sense of place refers to how people relate to or feel about the places where they live (Humon, 1992; Nanzer, 2004). Places can refer to locations of various sizes or boundaries, including a home, a neighborhood, a town or city, a meadow, a river canyon, a nation-state, or elsewhere. As noted by Worster \& Abrams (2005), there is more theoretical support than empirical evidence for the relationship between sense of place and environmentally responsible behaviors, but some research does support the link. For example, Uzzel, Pol and Badenas (2002) found personal identification with place to predict sustainability related behaviors among two neighborhood samples within England. While the theoretical case is strong, additional research is certainly needed to verify the relationship between a strong sense of place with environmentally responsible behaviors.

Hay (1998) explored sense of place in a developmental context, taking into account residential status, age stage, and adult pair bonding with a New Zealand sample. The residential status of the participants varied from highly mobile to deeply rooted, leading Hay to classify in terms of superficial (e.g., tourists), partial (e.g., holiday home owners), personal (recently established residents), ancestral (multigenerational residents), and cultural (indigenous peoples). Among other findings, his results demonstrated that sense of place varies with residential status, namely that those with a greater degree of rootedness, held the strongest sense of place. Multigenerational histories have been shown elsewhere to translate to a strong personal connection with place (see Opotow \& Brook, 2003 and Vitek \& Jackson, 1996).

Stability in a physical place also promotes social bonding and community relationship building (Twigger-Ross \& Uzzell, 1996, Armstrong, 2006, Hay, 2008). Further, Hay (1998) observed an interplay between the development of a sense of place and a pair bond, such that "pair bonds and place bonds intermesh in their significance, building upon each other” (p. 24). This finding corresponds with that of Kals, et al (1999), who showed that experiences of nature shared with a "significant other" result in a stronger development of emotional caring for nature. Hay continued by noting that developing a mature sense of place can foster feelings of security, stability, and belonging in much the same way as a mature intimate partner relationship. The transient and mobile nature for many people in contemporary society makes the establishment of mature relationships with people or place difficult. This poses a risk for continuity in one's own life story, thus posing a risk to community and societal cohesion (Hay, 1998). Hay concludes that ancestral and cultural foundations facilitate development of more mature bonds with place and community, delivering value for both individuals and society. The sentiment is echoed by Armstrong:

There's value in community, and there's value in family, and there's value in long-term relationships to place in relationships to each other. That's what builds strong community, and strong, healthy and vibrant people on a land, and a deeper understanding of how we need to be with each other, in order to be a certain way with the other relatives, the other living things that are on the land. That doesn't 
happen in one generation; that happens over many generations. As people know if they're third and fourth generation farmers or ranchers, or people connected to land in any other way, it becomes something much deeper. (personal communication, October, 21, 2007)

An important theme that has emerged here in discussing the restoration of human relationships with nature: such a development occurs in concert with and benefits from quality social dynamics. As social animals (Aronson, 1999), in seeking to rekindle the intimacy of our inherent relationship with nature, we must simultaneously succeed in mending the tears in the fabric of our human communities. Before discussing the subject of community restoration directly, it is worth noting that more research is warranted into the processes by which individuals develop a strong relationship with the environment. Further, research should seek to understand what other conditions are necessary for such identification to translate to enduring commitments towards ecologically responsible behaviors.

\section{Restoring Community}

When there's a loss of community, it's a transgenerational loss. It's a loss of the soul and of the spirit, and when that's combined with the loss of connection to the land, the loss of the ability to find yourself within that, the community finds itself in serious, serious trouble. So that, in terms of communities was the condition, and is the condition. (J. Armstrong, personal communication, October 21, 2007)

Cajete's (2000) chapter entitled, "Ecology of Native American Community," clearly outlines the seamlessness among the human, non-human and place relations that collectively define "community" in traditional Native societies. This perspective was echoed by multiple participants who expressed community as inclusive of relations with plants and animals and places. Even among non-Native populations, environmental context and the characteristics of place are not just a backdrop, but an integral part of socialization processes (Moser \& Uzzell, 2003). Appropriately, the overlap and interplay between social and environmental contexts was unavoidable in the preceding discussion on restoring relationships with the land. With this in mind, the following discussion about restoring community is heavily focused on human relations.

As exemplified in the opening quote, Armstrong vividly discussed the loss of community experienced by her people as a result of the "onslaught of violence" they experienced - not violence of physical force, but societal violence (see Iadacola \& Shupe, 1998) resulting in cultural disruption and severing of relationships. The experience of such violence and the subsequent breakdown in the fabric of the larger community was also spoken to by Conner and Craig, and alluded to by numerous other participants. A prime example of the societal violence experienced by Indian peoples is the Canadian and U.S. policies of removing children from their homes and into off-reservation boarding schools (Barker, 1997). As part of the systemic effort to "kill the Indian and save the child" they were treated as second-class citizens and taught that they needed to adopt white European cultural practices. Indian children were beaten for speaking their Native languages, and generally, mental, emotional, physical and sexual abuse was 
widespread (Barker, 1997). Armstrong explains that when these children returned home, many were broken, and many were self-destructive and committed suicide. They had no parenting skills, no knowledge of their culture and heritage, and an internalized selfloathing for being a Native person. Many continue to suffer and, subsequently, these traumatic effects continue to be experienced by successive generations (J. Armstrong, personal communication, October 21, 2007).

The difficulties observed among Native communities today can be traced back to the broader legacy of colonialism. As individuals continue to suffer from the wounds of these experiences the fabric of community has not yet been restored (Battiste, 2000; Duran \& Duran, 1995). Several interviews identified part of the challenge is to move beyond victimization and to build an understanding that there is value in the traditional ways of Native communities, value in Native knowledge, and value in Native cultures. Duran and Duran (1995) share this perspective and put forward a therapeutic strategy for working with indigenous populations that calls for ending perpetuation of the colonization pattern of relations that continue in applying Western models of therapy. Their approach includes emphasis on understanding historical context, as well as giving validation to Native epistemologies. Other emerging intervention strategies give similar emphasis to integrating aspects of traditional worldviews (e.g., Garrett \& Carroll, 2000; Moody, 1995). Evidence appears to support the argument that culturally sensitive approaches yield more effective results with ethnic minority populations (e.g., Hays \& Iwamasa, 2006).

For Native and non-Native peoples, understanding and evidence is growing that the health of overall community functioning is critical for the health of individuals (e.g., Adams, 1996; Robert, 1999). Bronfenbrenner's (1979) pioneering framework in The Ecology of Human Development achieved heightened awareness among the social sciences about the importance of community context in the developmental processes of children. While Bronfrenbrenner's conception of ecology and environment are exclusively of the human domain, noticeably lacking the natural environment as an aspect of "ecological" context, his perspective of nested systems and reciprocal dynamics are otherwise consistent with the theoretical perspectives put forward by this study and observed among the interviewee's perspectives. In short, Bronfenbrenner conceived of development occurring within context of nested systems, including the levels of microsystems (i.e., interpersonal interactions), mesosystems (i.e., organizational influences), exosystems (i.e., community influences), and macrosystems (i.e., cultural influences). Individual development occurs through direct interactions, or proximal processes. He emphasized the microsystem level as the predominant context that directly shapes individual development, but with an understanding that these interactions are shaped and informed by the larger contexts (Bronfenbrenner, 1979). Again, this perspective has helped influence both research and public policy to place greater weight on promoting community-level health as part of strategies to promote individual health (e.g., U.S. Department of Health and Human Services, 2000).

Seeking community health and individual well-being, including a sense of belonging, the naw'qinwixw process as outlined in Appendix $\mathrm{M}$ offers a compelling model for how to build and sustain community as a "whole healthy functioning organism.” Illustrated is a model that calls for a deliberate process of bringing together the diversity within a community to explore the issues and problems faced through 
dialogue. Inclusivity of all perspectives increases the likelihood of finding more effective solutions that work for everyone and reduces future conflicts. This governance process has worked effectively for the Syilx people for millennia, and may be a valuable model for the larger society to consider. Whether such a process is applicable on larger scalesas well as whether it would function effectively with the diversity characterizing modern secular society-are questions worthy of further consideration and future research. For discussion here, several aspects of the naw'qinwixw process suggest promise to the tool for creating and sustaining strong, healthy communities.

The work of Putnam $(1995 ; 2000)$ documents a U.S. society that has declined over recent decades in terms of community involvement, cohesiveness, and overall social capital. In using the phrase "social capital" here, I acknowledge its problematic aspects; from ambiguity and diffuseness of meaning to being a capitalism-based metaphor of social life (see Fine, 2001). Social capital "refers to features of social organization such as networks, norms, and social trust that facilitate coordination and cooperation for mutual benefit” (Putnam, 1995, p. 66). Social capital can be of two forms: bonding capital and bridging capital. The bonding form refers to the strength and closeness of relationships within groupings of people, while the bridging form refers to the strength and quality of relationships across groups of people (Putnam, 2000). Putnam outlines the benefits enjoyed by communities with strong social capital, including lower crime, greater citizen health, and more expedient and effective conflict resolution, among others (Putnam, 1995, 2000). The bonding and bridging dimensions are both salient in the naw'qinwixw process. Each of the four polarities, emblematically identified as mothers, fathers, innovators and elders, are encouraged to work together with like kind, but not exclusively, as engaging with the opposite polarity is essential to ensure overall community balance and wholeness (see Appendix M).

The naw'qinwixw process directly leverages community diversity and directly confronts the need for the development of trust and the pursuit of shared interests as a community. Putnam (2007) illustrates a contemporary U.S. society with many communities characterized by ethnic diversity and lowered trust both within groups and between groups. A variety of behaviors and attitudes reported by communities characterized by such diversity and low trust include: less likelihood of volunteering or donating to charity, greater distrust in local government, lower levels of confidence in one's own ability to influence political life, as well as fewer close friends and confidants and less happiness and overall lower perceived quality of life. Putnam's remedy to the separation experienced by people within communities begins with reducing the social distance among us and reconstructing our social identity to be more inclusive of others outside the immediate sphere of perceived sameness. Doing so would not eliminate preexisting personal identities, but would create an overarching identity that enhances the salience of common experiences and shared interests (Putnam, 2007).

While Native Americans are notably absent from Putnam's discourse, he effectively identifies a problem and offers some useful suggestion for alleviating that problem. Viewed from an indigenous perspective, Armstrong (2006; personal communication, October 21,2007 ) recognizes the problem of lack of community and its consequences. She is critical of contemporary society on a variety of levels, and cites our transience and subsequent scarceness of community as central to psychological, societal, and ecological problems observed today. By living transient lives, we are unable to 
establish a rooted connection to the Earth and we avoid emotionally bonding in relationships - two things that are central to being fully human. The resulting alienation from land and social disharmony leave people lacking concern for land or others, as they pursue individual interests without regard to the greater whole, their larger self (Armstrong, 2006).

For the Syilx (Okanagan), everyone is born into family and community, where they automatically belong. The community shares a bond of place, shares a history, and shares a future. The actions of each individual affect the community, so an important teaching emphasizes the community interests, followed by family, followed by individual interests. In living together, the people commune daily. To commune means to share, bond, create compassion, and develop the ability to emotionally process and respond to crises and celebrations as a whole community. The process of naw'qinwixw can be understood as a tool to facilitate bonding and the community's shared emotional response. Bonding with land and bonding with one another, the community is able to move forward as whole and healthy organism (Armstrong, 2006; J. Armstrong, personal communication, October 21, 2007).

Attempts to embody many of the basic principles outlined by Armstrong are seen in the movement to create intentional communities (e.g., Christian, 2003). Christian defines land-based intentional communities as "a group of people who have chosen to live with or near enough to each other to carry out their shared lifestyle or common purpose together" (2003, p. xvi). While only a small fraction of people in the U.S. live in intentional communities, they do appear to be in steady growth (Schaub, 2005). Research on intentional communities is limited, but evidence from an intergenerational intentional community suggest that participating elders defied the degenerative model of aging (Power, Eheart, Racine \& Karnik, 2007). Meaningful personal intergenerational relationships and purposeful engagement in the community appear to be key mechanisms helping these elders age well. The presence of intentional communities and their notability in terms of drawing a stark contrast with normative communities speaks to the absence of close-knit community throughout most of contemporary society.

While the building of strong communities is a laudable goal in itself, many intentional communities are formed with environmental restoration as a core purpose for their existence (Christian, 2003; Kozeny, 2005). Outside of intentional communities, Uzzell et al. (2002) demonstrated that social cohesion within a community was an important factor in predicting environmental behaviors. They argue that, "any long-term environmental behavior strategy has to be located in the relationships that exist between people in the community and the relationship between those people-individually and collectively_and their environment" (Uzzell et al., 2002, p. 50). As such, the strategies employed to restore the Earth need to be both sensitive to the relationships characterizing an existing community, as well as seek to promote cohesion among members of the community. This sentiment is echoed by Armstrong (2006), and embodied in the naw'qinwixw process, which bears all the trademarks of an effective process to promote social cohesion as practiced by people with a profound level of identification with place and other species.

At this stage of the discussion, the reader may be cognizant that despite categorization of major themes under different headings, no theme has been discussed exclusive of the other themes. For example, the preceding section our relationship with 
the land ultimately led to the importance of social dynamics and community, which eventually led back to the human relationship with the land. Also, the theme of identity has been unavoidable throughout. It speaks to the fundamental truth of holistic interconnectedness, and the awareness of this truth held by the project's participants. While Western science is maturing in its awareness and understanding of this truth, embodiment in the practice of science is still in its infancy. Fortunately, a relationship is budding between Western scientists and practitioners of traditional ecological knowledge (TEK). With further cooperation, TEK may benefit Western science in developing a more holistic understanding of phenomena.

\section{Cooperation: Focus on Bridging TEK and Science}

Those are the main issues: the loss of knowledge and the environmental crisis, [and] the need to bring science and TEK together for the mutual benefit to all of humanity. It is no longer a color issue, it's not an ethnic issue, it is a humanitarian issue at this time. (D. Martinez, personal communication, January 3, 2008)

One of the most recurrent themes of the project was that of the importance of working together to address the problems of today and to realize our hopes for tomorrow. While cooperative relationships are difficult to sustain, they often prove to generate optimal results for all parties involved (see Axelrod, 1997). The most commonly mentioned area calling for collaboration within the interviews was in regards to bridging the gap between science and traditional knowledge systems. In addition to overcoming differences in worldviews and methodological approaches, effectively bridging these two traditions requires building a trust undermined by a legacy of scientific exploitation of Native communities (see James, 2001). For more on the benefits, challenges, and opportunities of partnerships between indigenous knowledge systems and Western science, the reader is encouraged to see the special edition of Ecological Applications (2000), as well as James (2001). Discussion here is focused on the opportunity for indigenous systems of knowing to contribute to the development of Western science's more holistic understanding of phenomena.

Traditional ecological knowledge (TEK) can be viewed as the primary mode of knowledge transmission and acquisition that guided human action for thousands of years. Western science has supplanted TEK as the dominant model just within the last several hundred years. Western science is just beginning to mature in terms of its understanding that the reductionist method, while powerful in isolating discrete pieces of knowledge and generating specific applications, generates fragmented and incomplete understandings of the whole (e.g., Kline, 1996). Emerging scientific perspectives emphasize that more comprehensive understanding requires attention to whole systems (e.g., Altmann \& Koch, 1998) and that isolated disciplines that discount interrelationships among phenomena generate fragmented knowledge (e.g., Lattuca, 2001). Building links with TEK bears potential to contribute to rectifying the inadequacies of mainstream science, because TEK inherently is oriented toward interrelationships, and tends to approach problems with an understanding that the whole as greater than the sum of the parts (see Inglis, 1993). 
Among the obstacles to effectively bridging science and indigenous ways of knowing is the reluctance of Western science to accept the spiritual dimension of reality, even if only a subjective reality (Begay, 2003; Ford \& Martinez, 2000; see also Huntington, 2000). Indigenous practices of ceremonies and prayer for alignment with the natural world are conducted, "because there is no separation among indigenous peoples between spirit and matter. They are one and the same” (D. Martinez, personal communication, January 3, 2008). As such, the practicalities of day-to-day engagement with the land and caring for one's relatives (human and non-human) are conducted with respect, reverence, love and appreciation.

Western scientists unwilling or unable to see validity in a methodology informed by a spiritual belief discount the potential value and importance of such practices. For the skeptic, a spiritual truth need not require a metaphysical definition, but does assert that all things are profoundly connected as part of one larger whole (Capra \& SteindlRast, 1991). Empathy with such a perspective would grant concession to the meaningfulness of personal and cultural practices that honor and pay tribute to a spiritual reality, whether subjective or absolute. If skepticism morphed into a widespread embrace of a spiritual belief, then scientists (and those who rely on scientific findings in personal and policy decisions) would be less likely to pursue the historically celebrated, aggressive, quick-fix strategies. The goals of manipulation and control so often coupled with the practice of science would shift to goals of harmony and alignment. Several forces appear to be moving us in that direction, including the ever-increasing recognition of the consequences of our actions (e.g., MEA, 2005), and maturation of our theoretical conceptualizations - exemplified by systems perspectives - that move us away from the reductionist paradigm and place greater emphasis on relationships in environmental context (e.g., Capra, 1996), dynamic interrelationships over time (e.g., Gunderson \& Holling, 2002), and emergent phenomenon (e.g., Holland, 1999).

The cultural artifacts of modern society have been profoundly shaped by the reductionist paradigm of Western scientific methods. From the structure, process and content of our educational system to the structure and function of the macroeconomic system, the markings of reductionism are pervasive. The lives we lead within the structures of this paradigm are largely segmented lives-our work often disconnected from the needs of our local community; our homes arranged to serve single families rather than build community; our entertainment and recreation time to escape from the happenings of our daily life. The reordering and transformation of our cultural systems that is needed requires a holistic framework to guide our pursuit of knowledge and subsequent applications. Cooperative behavior among scientists and practitioners of TEK bears promise of aiding the development of such a framework and hastening the needed cultural transformation. 


\section{Culture: Enduring Identity}

[Sustainability has] got to start back at our culture. I mean, that's the foundation for our sustainability is our culture, our identity, who we are. (S. Yanity, personal communication, September, 20, 2007)

This project put forth the argument that achieving sustainability calls for change at all levels of culture, with culture consistent of multiple dimensions from visible artifacts and behaviors, to espoused values, to deeply held beliefs and assumptions (see Chapter IV). The less visible dimensions of culture, or the worldviews held by a culture's members, are foundational to the historical pattern of behavior of that culture, helping to explain present day circumstances and predict future behavioral trajectories. This project engaged Native leaders with interest in gaining perspective on the subject of sustainability outside an entrenched Western worldview. The results articulate potential cultural forms that establish sustainability as a preeminent value, and offer an alternative to mainstream Western culture.

The alternative articulated here addresses each level of culture: beliefs, values and behaviors. At the core level of beliefs, or assumptions about the nature of reality, several that have been highlighted and discussed include: a view of self as part of larger whole; understanding all things as intimately interconnected; and that humans hold responsibility for and play an important role in ecological health. Some of the prominent values include: holding respect for all things, having humility, engaging in reciprocity, and valuing the importance of all relations within a community. These beliefs and values provide the foundation for engaging in behaviors that include stewardship of the land, the bringing together of community, regular engagement in traditional cultural practices, and acting in partnership with others outside one's own immediate community. Taken together, a basic sketch is illustrated of potential cultural forms that could position humans on a more sustainable path.

Presenting these results as offering an alternative to the dominant Western culture need not imply co-optation of Native cultures. Rather, it is an invitation to consider the pragmatic value such beliefs, values and behavioral practices hold for potentially aligning our ways of life with the systems of nature within which we are embedded. Embracing such a framework, does not imply homogeneity across cultures as latitude is preserved for unique behavioral manifestations and variability among worldviews. Indeed, diverse manifestation is inevitable if indigenous perspectives are applied in our own unique contexts defined by history and place. It is not about telling the same stories, it is about telling stories with the same themes of respect, gratitude, humility, reciprocity, responsibility, reverence, harmony, balance and wholeness, for all people are subject to the same laws of nature.

As stated in the narrative, the ultimate test of a culture's worthiness of being passed onto future generations is the extent to which they serve the purpose of keeping in harmony and balance with natural law. As the indicators mount that the dominant cultural forms of today are failing this test of validity, alternatives such as that articulated here are worthy of consideration. With the proposed test of cultural validity in mind, consider a final example offered to: (a) illustrate one of the many challenges faced by modern tribes seeking to practice their traditional cultures; (b) draw attention to the 
pragmatic value of the featured tribe's cultural response; and, (c) highlight a unique aspect of the perspectives shared by the participating Native leaders regarding the meaning of sustainability.

The Stillaguamish tribe was part of the Point Elliott Treaty of 1855, nearly went extinct in the $20^{\text {th }}$ century and regained federal recognition in 1976. Today, they are relatively small with an enrollment of 237 (in 2003) and small scattered parcels of land. A few years ago, one of their ancestral burial grounds was unearthed by a highway construction project. Informed by their cultural values and beliefs, the event called for a ceremonial response to re-bury their ancestors in the proper way. Due to the history of cultural disruption, knowledge of how to proceed had been lost within the tribe. So they drew upon newly re-forged relations with neighboring tribes for guidance. The reburial effort called for materials to be gathered which were available only from the few places still resembling the native ecology of the area. The gathering of materials brought the community together, engaging youth, adults and elders in a shared activity towards a common purpose. This time together witnessed the sharing of stories and the practicing of their language. As a participant, Yanity experienced it as, "very spiritual and soulsearching," and helped him learn about himself (personal communication, September 20, 2007).

In this one example we see the tension between the age of modern development and the traditions of the past, the importance of sovereignty and gathering rights, the intimate link between a people and their land, the importance of land ownership to ensure land stewardship, the importance of a healthy ecosystem to provide needed resources, a tribe that was once near extinction restoring and recreating its cultural identity, and a value system and behavioral response informed by that identity.

The behavioral response of the Stillaguamish people here is motivated by a sense of responsibility to care for their ancestors to ensure their spirits have rest. As observers, even if we do not believe that the disruptions of ancestral remains disturb spirits or have any implications for the living's experience in the physical plane, we should still be able to appreciate the socio-cultural and pragmatic value of their response. By choosing a ceremonial behavioral response they were able to bring community together, be inclusive and respectful of elders as well as involve children (thus achieving intergenerational transfer of knowledge, language and values), build a relationship with the land, promote environmental stewardship, practice the skills needed for a subsistence based economy, get outside for fresh air and physical exercise, provide each individual with an experience where they feel a part of something larger than themselves, and deepen their understanding of who they are as human beings.

I have selected this example in closing because, on the surface, it may not appear to be directly relevant to the topic of sustainability as the dominant themes of environmental health and economic development are not featured prominently. However, when viewed through a holistic lens, one can see how this event occurs within the context of a much broader story playing out over a timeline nearly incomprehensible to most modern people. For a people to sustain themselves across this epic story, their life ways must serve to balance the needs of the present with responsibilities to the past, their source of identity and knowledge, as well as with responsibilities to future generations, the continuation of their identity. This is one of numerous examples that speak to sustainability as being about the enduring human experience within the circle of 
life. To talk of the interaction between the environment and the economy is to speak in abstract, segmented terms, while obscuring the human. The perspectives shared through this project highlight the human, with a holistic understanding of the human as an integral part of the natural world, bearing the responsibility to be a respectful participant in the web of life. For human identities to endure, our cultural forms must serve to reinforce this understanding and give guidance to appropriate modes of behavior to balance our stories with the story of the larger whole.

\section{Conclusion}

In Western society, words tend to diminish its meaning and the English language in my opinion tends to segment out a part of a whole. Sustainability taken from that perspective is too small a term from what I would consider to be an indigenous peoples perspective or a perspective of people who have had sustained and intimate contact with immediate environment for countless generations. We don't have such a term, because we live it. (L. Merculieff, personal communication, June 18, 2007)

Within the context of the unsustainable trajectory of collective human behavior, this project sought to engage Native leaders to better understand their thinking on the subject of sustainability. Currently, much of the mainstream consideration on this subject is focused on how to align business practices with a loosely defined idea of sustainability, and without serious reflection on the underlying assumptions of the paradigm from which those organizations were created and operate. Much of the academic conversation demonstrates these same shortcomings, and proceeds with minimal awareness of the deep interconnectedness of all phenomena. What is clear in the perspectives of the participating Native leaders is that we must reorient our goals to focus on mending our relationships with the planet, one another and ourselves, not matters of sustaining commerce in the current system of exploitation, power, control and amassing gross material wealth. The purpose of our educational and economic systems ought to be the facilitation of people making a life in a manner that promotes the welfare of not just a few, but of all life and the generations to come.

Further, use of the term sustainability must be grounded by a deep knowledge of who we are as human beings, including our profound connection with nature. From there we can draw wisdom in making the deliberate decisions regarding how to harmoniously sustain ourselves. Knowledge of who we are requires releasing our egos to recognize our humble yet integral place within the community of life. Wisdom in our decisions and actions depends on this humble and integral view of self and requires long-term perspective reaching generations beyond one's own lifetime. Such perspective demands a willingness to dissociate from many of the ill-conceived institutions and aspects of mainstream culture that consume not just the natural world but also our awareness of our inherent connection with all things.

As I heard an elder once say, everyone is spiritual whether they think of themselves as spiritual or not. For the agnostics and atheists uncomfortable with a metaphysical definition, spirituality can simply mean the totality and gestalt of who we are as living beings. Living a spiritual life then can be understood as experiencing the 
fullness of our mind and body, including the depth of our connection with and our inherent place within the Universe (Capra \& Steindl-Rast, 1991). In response to a question about how spirituality is related to sustainability, Maher struggled to even differentiate between the two (personal communication, August 17, 2007). Minthorn shared correspondence after reviewing his interview transcript: "I think it is a look into the soul of a person when that person tries to answer and define the notion of sustainability" (personal communication, September 13, 2007). It is with this depth of consideration that I believe we must pursue questions about sustainability. Casual use of the term will result in casual thinking complimented by actions and strategies that tinker at the margins of the problems. At hand is the need to address the core issue of bringing human modes of being back into balance and alignment with the larger patterns of life. It is a process of rediscovering the wholeness of our beings and embodying our unity with the Universe through the stories told by our individual and collective lives.

\section{Summary of Contribution and Looking Forward}

All I am saying is that if we want sustainability, we need a radical, very radical, realignment of the way we think. That's nothing new; there have been radicals in our history for 6,000 years saying that and I don't expect to be listened to anymore than they were. (W. Justin, personal communication, June 22, 2007)

Embracing the inherent subjectivity of science (Faulconer \& Williams, 1985; Fine \& Vanderslice, 1992; McGrath \& Johnson, 2003) and following in the tradition of social action research (e.g., Lewin, 1948), this project sought to contribute to the sustainability dialogue by giving voice to indigenous perspectives. The opening chapters of the dissertation and portions of the discussion present a critique of the dominant Western paradigm. Particularly problematic dimensions of the Western paradigm include: (a) the assumption that we are separate beings disconnected from one another and from nature; (b) valuing the individual above the group; and, (c) cultural artifacts and behaviors of a consumer-based economy, exploitive technologies, gross accumulation of material wealth, social and economic inequalities, and fragmented communities. Generally, dimensions of culture are perpetuated so long as they are seen as working well enough in meeting the group's challenges of external adaptation and internal integration to be considered valid (Schein, 1992). Chapter II argues that the dominant cultural forms are not working well enough to ensure long-term human adaptation and survival, and should therefore be considered invalid. As awareness of the crisis at hand grows, this project aimed to go beyond diagnosis of the problems to facilitate reflection on our underlying worldviews, and further, to offer hope and possibilities for the future.

The voices of the indigenous leaders presented here offer a rich and complex account of alternative ways of thinking about human modes of living within the broader context of the biosphere. Through a rigorous qualitative methodology I have represented my interpretation of their perspectives through the narrative summary, which was largely validated by the participants themselves. Their perspectives articulate a holistic conceptualization of the meaning of the term sustainability. The narrative also offers a hopeful vision for the future, and provides guidance for actions and strategies to move towards a sustainable future. As a whole, the narrative is an articulation of an alternative 
to the dominant Western paradigm. This alternative is characterized by: (a) the assumption of inherent and profound interconnectivity; (b) values of respect, harmony, reciprocity, and humility; and, (c) artifacts and behaviors such as inclusive dialogue, active stewardship of land, responsibility and care for others, and patterns of production and consumption sensitive to and aligned with ecological context.

The results in the form of the narrative summary demonstrate commonalities among a diverse group of indigenous leaders. Native and non-Native readers alike may find that they share much in common with the perspectives voiced here. The commonalities in human experience far outweigh the differences. That said, there is diversity across and within cultures, and every community has its own unique situational and historical context. Therefore, each reader is encouraged to evaluate the contents here relative to her or his own unique context. The degree to which the narrative transmits meaning, promotes understanding, gives direction, and inspires action for each reader is the ultimate measure of its validity.

In contrasting Western and Native paradigms, there is risk of conveying an oversimplified and over-generalized dichotomy. There is a broad spectrum of worldviews and many subcultures with greater or lesser degrees of identification with the generalized constructs of Western and Native. For the social action researcher focused on producing knowledge oriented toward change, this caveat is important in the dissemination of the project's content. Namely, the content will be differentially received by different individuals and subcultures. Among those seeking change and alternatives, there is greater opportunity for dialogue, collaboration and initiating action. Where there is greater resistance to change, the challenge of initiating and maintaining dialogue is correspondingly greater. While posing a greater challenge, resistant audiences are among the most important to engage in the process of realizing genuine and widespread change. For these dialogues to be constructive, participants must be sensitive to and respectful of the needs and perspectives of one another. Attempts to impose perspective will not facilitate the accommodation of mental models (i.e., worldviews), but rather harden and further entrench pre-existing positions. The process through which change is achieved is at least equally important as the goal.

As the changes necessary to sustain the human story are so extensive, there are few if any audiences that would not benefit from exposure to indigenous perspectives such as those represented here. This project made a humble effort to reach beyond an academic audience by developing a website and producing two videos aired on public access television (see Appendices $\mathrm{H}$ and I). Substantially more effort of this nature is needed to reach a broader audience and expand the dialogue.

Beyond communication through the media, academic forums, and community dialogue, it is important to remember that ultimately, sustainability is about our patterns of activity on the ground. In psychology, learning is often defined as stable changes in behavior as a result of experience (e.g., Gazzaniga \& Heatherton, 2006; Myers, 2001). From this perspective, learning is not about new conceptual understanding, it is about behavior change. The challenge is not only to achieve a conceptual understanding of sustainability. The real challenge is to embody that understanding in our modes of living. To be truly informed is to be in form - to be the physical manifestation of knowledge. We have not learned sustainability until we embody sustainability. We must live it. 


\section{REFERENCES}

Ackerman, L. S. (1984). The flow state: A new view of organizations and managing. In J. D. Adams (Ed.), Transforming work (pp. 114-137). Alexandria, VA: Miles River.

Adams, B. (1996). Building healthy communities. Report commissioned by the Pew Partnership for Civic Change. Charlottesville, VA: Suzanne W. Morse, Publisher. Retrieved April 20, 2008 from www.pew-partnership.org

Allen, P. G. (1979). Itani: It goes this way. In G. Hobson (Ed.), The remembered Earth (pp. 191-193). Albuquerque, NM: Red Earth Press.

Allen, P. G. (1979). The sacred hoop: A contemporary Indian perspective on American literature. In G. Hobson (Ed.), The remembered Earth (pp. 222-239). Albuquerque: Red Earth Press.

Altmann, G. \& Koch, W.A. (Eds.) (1998). Systems: New Paradigms for the Human Sciences. New York: Walter de Gruyter.

Aronson, E. (1999). The social animal ( $8^{\text {th }}$ Edition). Worth Publishers: New York.

Axelrod, R. M. (1997). The complexity of cooperation: Agent-based models of competition and collaboration. Princeton, N.J.: Princeton University Press,

Banister, P., Burman, E., Parker, I., Taylor, M., \& Tindall, C. (Eds.). (1994). Qualitative methods in psychology. Bristol, PA: Open University.

Barker, D. K. S. (1997). Kill the Indian, save the child: Cultural genocide and the boarding school. In D. Morison (Ed.), American Indian Studies: An Interdisciplinary Approach to Contemporary Issues (pp. 47-68), New York: Peter Lang Publishing Group.

Basile, G., \& Rosenblum, J. (2000). Walk this way. Forum for Applied Research and Public Policy, 15, 29-34.

Bateson, G. (1979). Mind and nature: a necessary unit. New York: Bantam Books.

Battiste, M. (Ed.) (2000). Reclaiming indigenous voice and vision. Vancouver, BC: UBC Press.

Baum, J. R., Locke, E. A., \& Kirkpatrick, S. A. (1998). A longitudinal study of the relation of vision and vision communication to venture growth in entrepreneurial firms. Journal of Applied Psychology, 83(1), 43-54.

Beattie, A., \& Ehrlich, P. (2001). Wild solutions: How biodiversity is money in the bank. New Haven, CT: Yale University Press. 
Begay, R.C. (2003). Science and spirituality. American Journal of Public Health, 93(3), 363-363.

Berg, B. (1998). Qualitative research methods for the social sciences. Boston: Allyn and Bacon.

Beringer, A. \& Martin, P. (2003). On adventure therapy and the natural worlds: Respecting nature's healing. Journal of Adventure Education \& Outdoor Learning, $\underline{3}(\underline{1})$, 29-39.

Berkes, F. (1999). Sacred ecology: Traditional ecological knowledge and resource management. Philadelphia, PA: Taylor and Francis.

Berkes, F., Colding, J., \& Folke, C. (2000). Rediscovery of traditional ecological knowledge as adaptive management. Ecological Applications, 10(5), 1251-1262.

Bertalanffy, L. V. (1968). General system theory: Foundations, development, applications. New York: George Braziller.

Bloom, H. (1995). The Lucifer principle: A scientific expedition into the forces of history. New York: The Atlantic Monthly Press.

Bluemink, E. (2006, November 14). Native revenues dominate corporations: With old problems gone, they are a force. [Electronic version]. Anchorage Daily News, p. T1.

Booth, A. (2003). We are the land: Native American views of nature. In H. Selin (Ed.), Nature across cultures: Views of nature and the environment in non-Western cultures (pp. 329-349). Amsterdam: Kluwer Academic Publishers.

Booth, A. L. (1996). Who am I? Who are you? The identification of self and other in three ecosophies. Trumpeter, 13(4), 1-10.

Boyd, D. K., Ream, R.R., Petscher, D.H. \& Fairchild, M.W. (1994). Prey taken by colonizing wolves and hunters in the Glacier National Park area. Journal of Wildlife Management, 58(2), 289.

Boyd, R. (1999). The coming of the spirit of pestilence: Introduced infectious diseases and population decline among Northwest Coast Indians, 1774-1874. Seattle: University of Washington Press.

Bronfenbrenner, U. (1979). The ecology of human development. Cambridge, MA: Harvard University Press. 
Brown, D., Dillard, J., \& \& Marshall, R. S. (2002). Triple bottom line: A business metaphor for a social construct. Barcelona: Department of Business Economics, Universitat Autonoma de Barcelona.

Buan, C. M., \& Lewis, R. (1991). The first Oregonians: An illustrated collection of essays on traditional lifeways, federal-Indian relations, and the state's Native people today. Portland, Oregon: Oregon Council for the Humanities.

Bury, B.R. (2004) Wildfire, Fuel Reduction, and Herpetofaunas across Diverse Landscape Mosaics in Northwestern Forests. Conservation Biology, 18 (4) , 968975

Buzan, T. (1991). The mind map book. New York: Penguin.

Cajete, G. (2000). Native science: Natural laws of interdependence. Santa Fe, New Mexico: Clearlight Publishers.

Camacho, D. E. (Ed.). (1998). Environmental injustices, political struggles: Race, class, and the environment. Durham, NC: Duke University Press.

Campaign 2000 (2006). Oh Canada! Too many children in poverty for too long ...: 2006 report card on child poverty in Canada. Retrieved November 25, 2006, from http://www.campaign2000.ca/rc/

Capra, F. \& Steindl-Rast, D. (1991). Belonging to the Universe. Harper: San Francisco.

Capra, F. (1996). The web of life: A new scientific understanding of living systems. New York: Anchor Books.

Capra, F. (2002). The hidden connections: A science for sustainable living. New York: Anchor Books.

Carey, A.B. \& Johnson, M.L. (1995). Small mammals in managed, naturally young, and old-growth forests. Ecological Applications, 5(2), 336-352.

Carroll, J. (Ed.) (1956). Language, thought, and reality: Selected writings of Benjamin Lee Whorf. Cambridge, Mass.: MIT Press.

Cedarholm, C. J., Johnson, D. H., Bilby, R. E., Dominguez, L. G., Garrett, A. M., Graeber, W. H., et al. (2000). Pacific salmon and wildlife - ecological contexts, relationships, and implications for management. Olympia, WA: Washington Department of Fish and Wildlife.

Cernetig, M. (2006, November 29). \$1 billion fails to land one treaty: First Nations pay the price while lawyers clean up. Vancouver Sun, p. A1. 
Chapin, M. (2004). A challenge to conservationists. World Watch, 17(6), 12-31.

Cheney, G. (2004, July 12). The corporate conscience and the triple bottom line. Accounting Today, Retrieved October 11, 2006, from http://www.webcpa.com/article.cfm?articleid=633\&pg=acctoday

Christian, D. (2003). Creating a life together: Practical tools to grow ecovillages and intentional communities. Gabriola Island, BC, Canada: New Society Publishers.

Cieurzo, C., \& Keitel, M. A. (1999). Ethics in qualitative research. In M. Kopala \& L. A. Suzuki (Eds.), Using qualitative methods in psychology (pp. 63-75). Thousand Oaks, CA: Sage.

Clayton W. Dumont, C.W., Jr. (1996). The demise of community and ecology in the Pacific Northwest: Historical roots of the ancient forest conflict. Sociological Perspectives, 39 (2), 277-300.

Clayton, S. \& Opotow, S. (Eds.) (2003). Identity and the natural environment: The psychological significance of nature. Cambridge, MA: MIT Press.

Clayton, S. (2003) Environmental identity: A conceptual and operational definition. In S. Clayton \& S. Opotow (Eds.) Identity and natural environment (pp. 45-66). Cambridge, MA: MIT Press.

Commission on Life Sciences (2000). Toxicological effects of methylmercury. Washington, D.C.: National Academy Press.

Cook, I., Crang, P., \& Thorpe, M. (1996). Biographies and geographies: consumer understandings of the origins of foods. British Food Journal, 100(3), 162-167.

Cook, N. D. (1998). Born to die: Disease and new world conquest, 1492-1650. Cambridge, UK: Cambridge University Press.

Craik, K. J. W. (1943). The nature of explanation. Cambridge, UK: Cambridge University Press.

Creedy, J. \& Wurzbacher, A.D. (2001), The economic value of a forested catchment with timber, water and carbon sequestration benefits, Ecological Economics, 38, 71-83.

Croizat, L. (1964). Space, time, form: The biological synthesis. Caracas, Venezuela: Published by the author.

D'Andrade, R. (2001). A cognitivist's view of the units debated in cultural anthropology. Cross-Cultural Research, 35(2), 242-257. 
Davis, M. H., Conklin, L., Smith, A. \& Luce, C. (1996). Effect of perspective taking on the cognitive representation of persons: A merging of self and other. Journal of Personality and Social Psychology, 70, 713-726.

Deloria, V.,Jr. (2000). The speculations of Krech. Worldviews: Environment, Culture, Religion, 4, 283-293.

DeNavas-Walt, C., Proctor, B. D., Lee, C. H., \& U.S. Census Bureau. (2006). Income, poverty, and health insurance coverage in the United States: 2005. Washington, DC: U.S. Government Printing Office.

Donovan, P. (2000). Managing for a whole forest, Patterns of Choice: A journal of people, land, and money. Retrieved March 15, 2008 from http://managingwholes.com/--sustainable-forestry.htm

Doppelt, B. (2003). Leading change toward sustainability: A change management guide for business, government, and civil society. Sheffield, UK: Greenleaf Publishing.

Duran, E. \& Duran, B. (1995) Native American postcolonial psychology. Albany, NY: State University of New York Press.

Eldredge, N. (1998). Life in the balance: Humanity and the biodiversity crisis. Princeton, NJ: Princeton University Press.

Elkington, J. (1998). Cannibals with forks: The triple bottom line of 21st century business. Gabriola Island, BC, Canada: New Society Publishers.

Ellingson, T. (2001). The myth of the noble savage. Berkeley: University of California Press.

Englin, J. \& Mendelsohn, R.(1991). A hedonic travel cost analysis for valuation of multiple components of site quality: The recreation value of forest management. Journal of Environmental Economics and Management, 21, 275-290.

Epstein, P. (1999). Global warming: Health and disease. Washington, D.C.: World Wildlife Fund.

Epstein, P., Diaz, H., Elias, S., Grabherr, G., Graham, N., Martens, W., MosleyThompson, E., \& Susskind, J. (1998). Biological and physical signs of climate change: Focus on mosquito borne diseases. Bulletin of the American Meteorological Society, 79, 409-417.

Erez, M., \& Gati, E. (2004). A dynamic, multi-level model of culture: From the micro level of the individual to the macro level of a global culture. Applied Psychology: An International Review, 53(4), 583-598. 
Faulconer, J. E., \& Williams, R. N. (1985). Temporality in human action: An alternative to positivism and historicism. American Psychologist, 40(11), 1179-1188.

Fine, B. (2001) Social capital versus social theory. Political economy and social science at the turn of the millennium. London: Routledge.

Fine, M. (1994). Working the hyphens: Reinventing self and other in qualitative research. In N. K. Denzin, \& Y. S. Lincoln (Eds.), Handbook of qualitative research (pp. 70-82). Thousand Oaks, CA: Sage.

Fine, M., \& Vanderslice, V. (1992). Qualitative activist research: Reflections on methods and politics. In F. Bryant, \& J. Edwards (Eds.), Methodological issues in applied social psychology: Social psychological applications to social issues (pp. 199218). New York: Plenum Press.

Ford, J. \& Martinez, D. (2000). Traditional ecological knowledge, ecosystem science, and environmental management. Ecological Applications, 10(5), 1249-1250.

Garrett, M.T. \& Carroll, J.J. (2000). Mending the broken circle: Treatment of substance dependence among Native Americans. Journal of Counseling \& Development, 78(4), 379-388.

Gazzaniga, M.S. \& Heatherton, T. F. (2006). Psychological Science (2 ${ }^{\text {nd }}$ ed.). New York: W. W. Norton.

Gende, S. M., Edwards, R. T., Willson, M. F., \& Wipfli, M. S. (2002). Pacific salmon in aquatic and terrestrial ecosystems. BioScience, 52(10), 917-928.

Gentner, D. \& Goldwin-Meadow, S. (Eds.) (2003). Language in mind: advances in the study of language and thought. Massachusetts: MIT Press.

Gillis, W. R., Textor, R. B., \& Mitchell, M. (2006). The good road forward: A ten-year composite vision shared by leaders of 15 Native American tribes in the Owens and Central Valleys of California. Pullman, WA: Washington State University Center to Bridge the Digital Divide.

Glaser, G. (2008, February 16). Well, doctor, I have this recycling problem. New York Times. Retrieved February 18, 2008 from http://www.nytimes.com

Goldstein, B. E. (2002). Sensation and perception (6th ed.). Pacific grove, CA: Wadsworth-Thomason Learning.

Goudie, A. (1990). The human impact on the natural environment (3rd ed.). Cambridge, MA: The MIT Press. 
Guba, E. G., \& Lincoln, Y. S. (1989). Fourth generation evaluation. Newbury Park, CA: Sage.

Gunderson, L. H. \& Holling, C.S. (2002). Panarchy: Understanding transformations in human and natural systems. Washington, D.C.: Island Press.

Gwartney, J., \& Lawson, R. (2002). Economic freedom of the world: 2002 annual report. Vancouver BC, Canada: The Fraser Institute.

Hardin, G. (1968). The tragedy of the commons. Science, 162, 1243-1248.

Harkin, M. E. \& Lewis, D.L. (Eds.) (2007). Native Americans and the environment: Perspectives on the ecological Indian. Lincoln: University of Nebraska Press.

Hart, S. L. (1995). A natural-resource-based view of the firm. Academy of Management Review, 20(4), 986-1014.

Hartig, T., Mang, M., \& Evans, G.W. (1991). Restorative effects of natural environment experiences. Environment and Behavior, 23 (1), 3-26.

Haspelmath, M. (2001). The European linguistic area: Standard Average European. In, Language typology and language universals, vol. 20.2, (pp. 1492-1510). Berlin: Mouton de Gruyter.

Hassan, R., Scholes, R., Ash, N., \& Condition and Trends Working Group. (2005). Summary: Ecosystems and their services around the year 2000. In R. Hassan, R. Scholes \& N. Ash (Eds.), Ecosystems and human well-being: Current state and trends: Findings of the condition and trends working group of the Millennium Ecosystem Assessment [Electronic version] (p. 2). Washington, DC: Island Press.

Hawken, P. (1993). The ecology of commerce: A declaration of sustainability. New York: Harper Books.

Hay, R. (1998). Sense of place in developmental context. Journal of Environmental Psychology, 18, 5-29.

Hays, P.A. \& Iwamasa, G.Y. (Eds.) (2006). Culturally responsive cognitive-behavioral therapy: Assessment, practice, and supervision. Washington, DC: American Psychological Association.

Hilderbrand, G. V., Farley, S. D., Schwartz, C. C., \& Robbins, C. T. (2004). Importance of salmon to wildlife: Implications for integrated management. Ursus, 15(1), 1-9.

Hobbs, H. M. (2004a). Sustainable water management - the New Zealand perspective. High-Level Segment of the 12th Session of the United Nations Commission on Sustainable Development. Retrieved on October 27, 2006, from 
http://www.mfat.govt.nz/Media-and-publications/Media/MFAT-speeches/2004/028-April-2004.php

Hobbs, H. M. (2004b). Meeting targets, goals and timetables. High-Level Segment of the 12th Session of the United Nations Commission on Sustainable Development. Retrieved on October 27, 2006, from http://www.mfat.govt.nz/Media-andpublications/Media/MFAT-speeches/2004/0-28-April-2004a.php

Hofrichter, R. (Ed.). (1993). Toxic struggles: The theory and practice of environmental justice. Philadelphia: New Society Publishers.

Hogg, M. A., Williams, K. D. (2000). From I to we: Social identity and the collective self. Group Dynamics: Theory, Research, and Practice, 4(1), 81-97.

Holland, J. H. (1999). Emergence: From chaos to order. New York: Basic Books.

House, J., Brovkin, V., Betts, R., Constanza, B., Dias, M., \& Holland, B. (2005). Climate and air quality. In R. Hassan, R. Scholes \& N. Ash (Eds.), Ecosystems and human well-being: Current state and trends: Findings of the Condition and Trends Working Group of the Millennium Ecosystem Assessment [Electronic version] (pp. 360-361). Washington, DC: Island Press.

Houser, S., Teller, V., MacCracken, M., Gough, R., \& Spears, S. (2000). The potential consequences of climate variability and change for Native peoples and homelands. Climate change impacts on the United States: The potential consequences of climate variability and change (pp. 351-377). Cambridge University Press: Cambridge, UK.

Howard, A., \& Coombe, D. (2006). National level intentional change: A story of two countries. Journal of Management Development, 25(7), 732-742.

Howard, R. A., \& Matheson, J. E. (1981). Influence diagrams. In R.A. Howard, \& J.E. Matheson (Eds.), Readings on the principles and applications of decision analysis. Menlo Park, CA: Strategic Decisions Group.

Huberman, A. M., \& Miles, M. B. (1994). Data management and analysis methods. In N. K. Denzin, \& Y. S. Lincoln (Eds.), Handbook of qualitative research (pp. 428444). Thousand Oaks, CA: Sage.

Hughes, T., Baird, A., Bellwood, D., Card, M., Connolly, S., Folke, C., et al. (2003). Climate change, human impacts, and the resilience of coral reefs. Science, 301(5635), 929-933.

Humon, D. M. (1992). Community attachment: Local sentiment and sense of place. In I. Altman, \& S.M. Low (Eds.), Place attachment (pp. 253-278). New York: Plenum Press. 
Hunn, E, Johnson, D, Russell, P, Thornton, T. (2003). Huna Tlingit traditional environmental knowledge, conservation, of a "wilderness” park. Cultural Anthropology, 44, 79-103.

Huntington, H. P. (2000). Using traditional ecological knowledge in science: Methods and applications. Ecological Applications, 10 (5), 1270-1274.

Iadacola, P. \& Shupe, A. (1998). Violence, inequality, and human freedom. New York: General Hall Publishers.

Inglix, J. T. (ed.) (1993). Traditional ecological knowledge: Concepts and cases. Ottawa: International Program on Traditional Ecological Knowledge and International Development Research Centre.

Isham, J. \& Waage, S. (2007). Ignition: What you can do to fight global warming and spark a movement. Washington, DC: Island Press.

James, K. (2000). American Indians, science, and technology. Social Science Computer Review, 18, 196-213.

Jantsch, E. (1980). The self-organizing universe: Scientific and human implications of the emerging paradigm of evolution (1st ed.). New York: Pergamon Press.

Armstrong, J. (2006). Community: "Sharing one skin." In J. Mander \& V. Tauli-Corpuz (Eds.), Paradigm Wars: Indigenous Peoples' Resistance to Economic Globalization (pp. 29-36). Los Angeles, CA: University of California Press.

Johnson-Laird, P. N. (2000). The current state of the mental model theory. In GarcíaMadruga, J., Carriedo, M, and Gonsález-Labra, M.J. (Ed.), Mental models in reasoning (pp. 17-40). Madrid: UNED.

Kals, E., Schumacher, D., \& Montada, L. (1999). Emotional affinity towards nature as a motivational basis to protect nature. Environment \& Behavior, 31 (2), 178-202.

Kals, E. \& Maes, J. (2002). Sustainable development and emotions. In P. Schmuck \& W.P. Schultz (Eds.), Psychology of Sustainable Development (pp. 97-122). Boston, MA: Kluwer Academic Publishers.

Katz, D. \& Kahn, R. L. (1978). Social psychology of organizations. New York: John Wiley \& Sons.

Kitayama, S. (2002). Culture and basic psychological processes: Toward a system view of culture. Psychological Bulletin, 128, 189-196.

Kline, S.J. (1996). The powers and limitations of reductionism and synoptism. Bulletin of Science, Technology \& Society, 16, 3, 129-142. 
Kluckhohn, F. R. \& Strodtbeck, F. L. (1961). Variations in value orientations. Evanston, IL: Row, Peterson and Company.

Korten, D. (2001). When corporations rule the world. San Francisco, CA: BerrettKoehler Publishers.

Korten, D. C. (2006). The great turning: From empire to Earth community. San Francisco, CA: Berrett-Koehler.

Kozeny, G. (2005). In community, intentionally. In Communities directory: A comprehensive guide to intentional communities and cooperative living (pp. 1217). Rutledge, Missouri: The Fellowship for Intentional Community.

Krech, S. (1999). The ecological Indian: Myth and history. New York: W.W. Norton \& Company.

Kroeber, A. L. (1934). Native American population. American Anthropologist, New Series, 36(1), 1-25.

Kroeber, A. L., \& Kluckhohn, C. (1963). Culture: A critical review of concepts and definitions. New York: Random House.

Kuhn, T. S. (1970). The structure of scientific revolutions (2nd ed.). Chicago, IL: University of Chicago Press.

Kuhnlein, H.V. \& Receveur, O. (1996). Dietary change and traditional food systems of indigenous peoples. Annual Review of Nutrition, 16, 417-442.

LaDuke, W. (1993). A society based on conquest cannot be sustained: Native peoples and the environmental crisis. In Richard Hofrichter (Ed.), Toxic struggles: Theory and practicve of environmental justice (pp. 98-106). Philadelphia: New Society Publishers.

Ladum, A. M. (2006). War, peacebuilding, and concepts of relationship: Women in the Sierra Leonean civil war. Unpublished masters thesis, Portland State University, Portland, Oregon.

Laszlo, E. (1972). The systems view of the world: The natural philosophy of the new developments in the sciences. New York: G. Braziller.

Lattuca, L. R. (2001). Creating interdisciplinarity: Interdisciplinary research and teaching among college and university faculty. Nashville, TN: Vanderbilt University Press.

Leite, J. C. (2005). The World Social Forum: Strategies of resistance. Chicago, IL: Haymarket Books 
Lendaris, G. G. (1986). On systemness and the problem solver: Tutorial comments. IEEE Transaction on Systems, Man, \& Cybernetics, (July), 603-610.

Levin, I. M. (2000). Vision revisited: Telling the story of the future. The Journal of Applied Behavioral Science, 36, 91-107.

Lewin, K. (1948). Resolving social conflicts: Selected papers on group dynamics. New York: Harper.

Lincoln, Y. S., \& Guba, E. G. (1985). Designing a naturalistic inquiry. Y. S. Lincoln \& E.G. Guba (Eds.), Naturalistic inquiry (pp. 221-249). Newbury Park, CA: Sage.

Little Bear, L. (2000). Jagged worldviews colliding. In M. Battiste (Ed.), Reclaiming indigenous voice and vision (pp. 77-85). Vancouver: UBC Press.

Lyons, E. \& Breakwell, G. M. (1994). Factors predicting environmental concern and indifference in 13- to 16-year-olds. Environment and Behavior, 26, 223-238.

Mace, G., Masundire, H., Baillie, J., Ricketts, T., Brooks, T., \& Hoffman, M. (2005). Biodiversity. In R. Hassan, R. Scholes \& N. Ash (Eds.), Ecosystems and human well-being: Current state and trends: Findings of the Condition and Trends Working Group of the Millennium Ecosystem Assessment [Electronic version] (p. 96). Washington: Island Press.

Maller, C., Townsend, M., Brown, P., \& St Leger, L. (2002). The health benefits of contact with nature in a park context: A review of current literature. Melbourne: Deakin University and Parks Victoria.

Malmqvist, B., \& Rundle, S. (2002). Threats to the running water ecosystems of the world. Environmental Conservation, 29(2), 134-153.

Manson, S.M., \& Trimble, J.E., (1982). American Indian and Alaska Native communities: Past efforts, future inquiries. In L.R. Snowden (Ed.), Reaching the underserved: Mental health needs of neglected populations. Beverly Hills, CA: Sage Publications.

Marshall, C., \& Rossman, G. B. (1999). Designing qualitative research (3rd ed.). Thousand Oaks, CA: Sage.

McGrath, J. \& Johnson, B. (2003). Methodology makes meaning: How both qualitative and quantitative paradigms shape evidence and its interpretation. In P. M. Camic, J.E. Rhodes, \& L. Yardley (Eds.), Qualitative research in psychology: Expanding perspectives in methodology and design (pp. 31-48). Washington, DC: American Psychological Association. 
Meadows, D. (1986). Poverty causes population growth causes poverty. Sustainability Institute, The Donella Meadows Archive. Retrieved November 17, 2006 from http://www.sustainabilityinstitute.org/dhm_archive/index.php?display_article=vn 126manupured

Meadows, D. (1999). Leverage points: Places to intervene in a system. Hartland, Vermont: The Sustainability Institute.

Merchant, C. (1983). The death of nature: Women, ecology and the scientific revolution. San Francisco: HarperSanFrancisco.

Merrick, E. (1999). An exploration of quality in qualitative research: Are reliability and validity relevant? In M. Kopala \& L.A. Suzuki (Ed.), Using qualitative methods in psychology (pp. 25-36). Thousand Oaks, CA: Sage.

Michaels, C. A., \& Lopez, E. C. (2005). Collaboration and consultation in transition planning: Introduction to the mini-theme [Electronic version]. Journal of Educational \& Psychological Consultation, 16(4), 255-261.

Millennium Ecosystem Assessment. (2005). Ecosystems and human well-being [electronic version]. Washington: Island Press.

Miller, J. G. (1978). Living systems. New York: McGraw-Hill.

Mohai, P., \& Bryant, B. (1992). Environmental injustices: Weighing race and class as factors in the distribution of environmental hazards. University of Colorado Law Review, 63, 927.

Montero, M. (2001). From action and reflection to critical psychology. Critical Psychology, 1(1), 84-89.

Moody, J. (1995). Art therapy: Bridging barriers with Native American clients. Art Therapy, 12(4), 220-226.

Morgan, D. L. (1997). Conducting and analyzing focus groups. Thousand Oaks, CA: Sage.

Morse, J. M. (1991). Strategies for sampling. In J. M. Morse (Ed.), Qualitative research: A contemporary dialogue (pp. 127-145). Newbury Park, CA: Sage.

Moser, G., \& Uzzell, D. L. (2003). Environmental psychology. In T. Millon \& M. J. Lerner (Eds.), Comprehensive handbook of psychology: Volume 5.Personality and social psychology. New York: John Wiley.

Myers, N. (1979). The sinking arc: A new look at the problem of disappearing species. Oxford: Pergamon Press. 
Myers, D. (2001). Psychology (6 ${ }^{\text {th }}$ ed.). New York: Worth Publishers.

Nattrass, B., \& Altomare, M. (2002). Dancing with the tiger. Gabriola Island, BC: New Society Publishers.

Neihardt, J. G. (1932). Black Elk speaks. Lincoln, NE: University of Nebraska Press.

Neumann, C. M., Kauffman, K. W., \& Gilroy, D. J. (1997). Methylmercury in fish from Owyhee Reservoir in Southeast Oregon: Scientific uncertainty and fish advisories. The Science of the Total Environment, (201), 205-214.

Novak, J. D. (1991). Clarify with concept maps: A tool for students and teachers alike. The Science Teacher, 58, 45-49.

Novak, T. (1998). Why try to save wild salmon? (No. EM 8722). Corvalis, OR: OSU Extension Services.

Novak, J. D., \& Gowin, D. B. (1984). Learning how to learn. New York: Cambridge University Press.

Olson, D. M., Dinerstein, E., Wikramanayake, E. D., Burgess, N. D., Powell, G. V. N., Underwood, E. C., et al. (2001). Terrestrial ecoregions of the world: A new map of life on earth [Electronic version]. Bioscience, 51(11), 933.

Opotow, S., \& Brook, A. (2003). A. Identity and exclusion in rangeland conflict. In S. Clayton \& S. Opotow (Eds.), Identity and the natural environment: The psychological significance of nature. Cambridge, MA: MIT Press.

Oregon Natural Resource Council (2002). Bush unveils plan to boost logging Oregon's public forests. Retrieved May, 20, 2004 from www.onrc.org/press/044.bushplan.html

Orr, D. (1992) Ecological literacy: Education and the transition to a postmodern world. Albany, NY, State University of New York Press.

Patton, M. Q. (1990). Qualitative evaluation and research methods (2nd ed.). Newbury Park, CA: Sage.

Patton, M. Q. (1999). Enhancing the quality and credibility of qualitative analysis. Health Services Research, 34, 1189-1208.

Patton, M. Q. (2002). Qualitative evaluation and research methods (3rd ed.). Newbury Park, CA: Sage.

Pennybacker, M. (2000). The first environmentalists. The Nation, 270(5), 229-31. 
Peters, W. (2006). Statement made to the United Nations General Assembly, sixty-first session, general debate on 22 September 2006. Retrieved November 8, 2006, from http://www.nzmissionny.org/minister.htm

Phillips, J. L., Ory, L., Talbot, A. (2000). An anadromous salmonid recovery in the Umatilla River Basing, Oregon: A case study. Journal of the American Water Resources Association 36 (6), 1287-1308.

Piaget, J. (1952). The origins of intelligence in children. (M. Cook Trans.). New York: International Universities Press, Inc.

Piaget, J. (1983). Piaget's theory. In P. Mussen (Ed.), Handbook of child psychology (4th ed.). New York, New York: Wiley.

Powell, J.W. (1891). Eleventh annual report of the United States Geological Survey to the Secretary of the Interior, 1889-90, Part II-Irrigation. Retrieved October 27, 2006 from http://onlinepubs.er.usgs.gov/djvu/AR/ar_11_2.djvu

Power, M.B., Eheart, B.K., Racine, D. \& Karnik, N.S. (2007). Aging well in an intentional intergenerational community: Meaningful relationships and purposeful engagement. Journal of Intergenerational relationships, 5 (2), 7-25.

Pryor, A., Carpenter, C., Townsend, M. (2005). Outdoor education and bush adventure therapy: A socio-ecological approach to health and wellbeing. Australian Journal of Outdoor Education, 9(1), 3-13.

Putnam, R. (2007). E Pluribus Unum: Diversity and community in the twenty-first century: The 2006 Johan Skytte Prize Lecture. Scandinavian Political Studies, 30 (2), 137-174. Retrieved April 3, 2008, from http://www.blackwellsynergy.com/doi/abs/10.1111/j.1467-9477.2007.00176.x

Putnam, R. D. (2000). Bowling Alone: The Collapse and Revival of American Community. New York: Simon \& Schuster.

Putnam, R.D. (1995). Bowling alone: America's declining social capital. Journal of Democracy, 6 (1), 65-78.

Rands, G., Ribbens, B., Casagrande, D., \& McIlvaine-Newsad, H. (2007). Envisioning an ecologically sustainable society. An ideal type and an application. In S. Sharma (Ed.), Organizations and the sustainability mosaic: Crafting long-term ecological and societal solutions (pp. 32-56). Northampton, MA, US: Edward Elgar Publishing.

Rapaport, A. (1986). General systems theory: Essential concepts and applications. Cambridge, MA: Abacus Press. 
Rees, W. E. (1996). Revisiting carrying capacity: Area-based indicators of sustainability. Population and Environment, 17, 225-239.

Rees, W., \& Wackernagel, M. (1994). Ecological footprints and appropriated carrying capacity: Measuring the natural capital requirements of the human economy. In A. Jansson, M. Hammer, C. Folke \& R. Costanza (Eds.), Investing in natural capital: The ecological economics approach to sustainability (pp. 362-390). Washington: Island Press.

Richardson, T. E. (2002). Introduction. In J. T. E. Richarson (Ed.), Handbook of qualitative research methods for psychology and the social sciences (pp. 3-11). Oxford, UK: BPS Blackwell.

Ringquist, E. J. (2000). Environmental justice: Normative concerns and empirical evidence. In N. J. Vig \& M. E. Kraft (Eds.), Environmental policy (4th ed., pp. 232-256). Washington, DC: Congressional Quarterly Press.

Ritchie, J. (2003). The applications of qualitative methods to social research. In J. Ritchie \& J. Lewis (Eds.), Qualitative research practice: A guide for social science students and researchers (pp. 24-46). London: Sage.

Ritchie, J., Lewis, J., \& Elam, G. (2003). Designing and selecting samples. In J. Ritchie \& J. Lewis (Eds.), Qualitative research practice: A guide for social science students and researchers (pp. 77-108). London: Sage.

Ritchie, J., Spencer, L., \& O’Connor, W. (2003). Carrying out qualitative analysis. In J. Ritchie \& J. Lewis (Eds.), Qualitative research practice: A guide for social science students and researchers (pp. 219-262). London: Sage.

Robert, K. H. (1997). A compass for sustainable development. International Journal of Sustainable Development and World Ecology, (4), 79-92.

Robert, S.A. (1999). Socioeconomic position and health: The independent contribution of community socioeconomic context. Annual Review of Sociology, 25(1), 489-517.

Roberts, H. (Ed.). (1981). Doing feminist research. London: Routledge and Kegan Paul.

Robinson, E. (2004, April 13). A decade of compromise: Timber towns struggle under forest plan. The Columbian, pp. A1, A4.

Rosenberg, M. (1981). The self-concept: social product and social force. In M. Rosenberg \& R. H. Turner (Eds.), Social psychology: Sociological perspectives (pp. 593-624). New York: Basic Books.

Rosenblum, J. (2000). A deeper look at the system conditions. The Natural Step Newsletter, 1, 8-11. 
Roszak, T., Gomes, M.E., Kanner, A.D. (Eds.) (1995). Ecopsychology: Restoring the earth healing the mind. San Francisco: Sierra Club Books.

Ruckelshaus, W. (1989). Toward a sustainable world. Scientific American, (261), 166174.

Salmon, E. (2000) Kincentric ecology: Indigenous perceptions of the human-nature relationship. Ecological Applications, 10 (5), 1327-1332.

Sandelowski, M. (1995). Sample size in qualitative research. Research in Nursing \& Health, 18(2), 179-183.

Schaub, L. (2005). State of the communities movement. In Communities directory: A comprehensive guide to intentional communities and cooperative living (pp. 1822). Rutledge, Missouri: The Fellowship for Intentional Community.

Schein, E. (1992). Organizational culture and leadership. San Francisco: Jossey-Bass.

Schilizzi, S. (2002). Triple bottom line accounting: How serious is it? Connections. Retrieved October 11, 2006 from http://www.agrifood.info/10pub_conn_Win2002.htm

Schnaiberg, A. (1980). The environment: From surplus to scarcity. New York: Oxford University Press.

Schnaiberg, A., \& Gould, K. A. (1994). Environment and society: The enduring conflict. New York: St. Martin's Press.

Schouw, J. F. (1823). Grunzuge einer allgemeninen pflanzengeiographie. Berlin: Reimer.

Schultz, P. W. (2000). Empathizing with nature: Toward a social-cognitive theory of environmental concern. Journal of Social Issues, 56, 391-406.

Schultz, P. W. (2001). The structure of environmental concern: Concern for self, other people, and the biosphere. Journal of Environmental Psychology, 21(4), 327-339.

Schultz, P. W. (2002). Inclusion with nature: Understanding human-nature interactions. In P. Schmuck, \& P. W. Schultz (Eds.), The psychology of sustainable development (pp. 61-78). New York: Kluwer.

Schweder, R. A., \& Levine, R. A. (1984). Culture theory: Essays on mind, self and emotion. New York: Cambridge University Press.

Senge, P. M. (1990). The fifth discipline: The art and practice of a learning organization. New York: Doubleday. 
Skolimowski, H. (1981). Eco-philosophy: Designing new tactics for living. Boston: Marion Boyars.

Smith E, Wishnie, M. (2000). Conservation and subsistence in small-scale societies. Annual Review of Anthropology, 29:493-524.

Smith, H S. \& Anderson,J.S. (2001). Managing tribal assets. In K. James (Ed.), Science and Native American communities: Legacies of pain, visions of promise (pp. 8391). Lincoln and London: University of Nebraska Press.

Smith, J. (1995). Semi-structured interviewing and qualitative analysis. In J. Smith, R. Hare \& L. Van Langenhove (Eds.), Rethinking methods in psychology (pp. 9-27). London: Sage Publications

Snape, D., \& Spencer, L. (2003). The foundations of qualitative research. In J. Ritchie, \& J. Lewis (Eds.), Qualitative research practice: A guide for social science students and researchers (pp. 1-23). London: Sage.

Spencer, L., Ritchie, J., \& O’Connor, W. (2003). Analysis: Practices, principles and processes. In J. Ritchie, \& J. Lewis (Eds.), Qualitative research practice: A guide for social science students and researchers (pp. 199-218). London: Sage.

Sterman, J. D. (2000). Business dynamics: Systems thinking and modeling for a complex world. Boston: Irwin McGraw-Hill.

Stiles, W. B. (1993). Quality control in qualitative research. Clinical Psychology Review, 13, 593-618.

Stokols, D. (1996). Translating social ecological theory into guidelines for community health promotion. American Journal of Health Promotion, 10, 282-298.

Strandberg, C. H. (1971). Water pollution. In G. H. Smith (Ed.), Conservation of natural resources (4th ed., pp. 189-219). New York: Wiley.

Stryker, S. (1968). Identity salience and role performance: The importance of symbolic interaction theory for family research. Journal of Marriage and the Family, 30, 558-64.

Stryker, S. (1980). Symbolic interactionism: A social structural version. Palo Alto, CA: Benjamin/Cummings.

Swainson, W. (1835). A treatise on the geography and classification of animals. London: Longman, Green.

Swimme, B., \& Berry, T. (1992). The universe story: From the primordial flaring forth to the Ecozoic era. San Francisco: Harper Collins. 
Terborgh J. (1999). Requiem for nature. Washington, DC: Island/Shearwater

Textor, R. B. (1980). A handbook on ethnographic futures research. Stanford, CA: Cultural and Educational Futures Research Project, Stanford University.

Textor, R. B. (1990a). "Methodological appendix.” In S. Ketudat (Ed.), The middle path for the future of Thailand: Technology in harmony with culture and environment (pp. 135-152). Honolulu: Institute for Culture and Communication, East-West Center.

Textor, R. B. (1990b). "Introduction." In S. Ketudat (Ed.), The middle path for the future of Thailand: Technology in harmony with culture and environment (pp. xxii-xlv). Honolulu: Institute for Culture and Communication, East-West Center.

Tolman, E. C. (1948). Cognitive maps in rats and man. Psychological Review, 55, 189208.

Tourtellot, J. B. (2005). Destinations scorecard: National parks. National Geographic Traveler, July/August 2005. Retrieved February 15, 2008 from: http://www.nationalgeographic.com/traveler/features/nprated0507/nprated.html

Travis, J. (2003). Climate change and habitat destruction: A deadly anthropogenic cocktail. Biological Sciences, 270(1514), 467-473.

Triandis, H. C. (1972). The analysis of subjective culture. New York: Wiley-Interscience.

Triandis, H. C. (1980). Values, attitudes, and interpersonal behavior. In J. H.E. Howe, \& M.M. Page (Eds.), Nebraska symposium on motivation,1979 (pp. 195-259). Lincoln, NE: University Of Nebraska Press.

Triandis, H. C., Kurowski, L. L., \& Gelfand, M. J. (1994). Workplace diversity. In M. D. Dunnette, \& L. M. Hough (Eds.), Handbook of industrial organizational psychology (pp. 769-827). Palo Alto, CA: Consulting Psychologists Press.

Trimble, J. E. (1976). Value differences among American Indians: Concerns for the concerned counselor. In P. B. Pedersen, W. J. Lonner \& J. G. Draguns (Eds.), Counseling across cultures (pp. 65-81). Honolulu: University of Hawaii Press.

Trimble, J. E., \& Thurman, P. J. (2002). Ethnocultural considerations and strategies for providing counseling services to Native American Indians. In P. B. Pedersen, J. G. Draguns, W. J. Lonner \& J. E. Trimble (Eds.), Counseling across cultures (5th ed.). Thousand Oaks, CA: Sage.

Trimble, S. \& Nabhan, G. P. (1994). The geography of childhood. Boston, MA: Beacon Press). 
Turner, J. C, Hogg, M.A., Oakes, P.J., Reicher, S.D., and Wetherell, M.S. (1987). Rediscovering the social group: A self-categorization theory. New York: Basil Blackwell.

Twigger-Ross, C. L. \& Uzzell, D. L. (1996). Place and identity processes. Journal of Environmental Psychology 16, 205-220.

U.S. Department of Health and Human Services (2000). Healthy people 2010:

Understanding and improving health, 2nd ed. Washington, DC: U.S. Government Printing Office.

Union of Concerned Scientists. (1992). World scientists' warning to humanity. Retrieved 9/21/06, from http://www.ucsusa.org/ucs/about/1992-world-scientists-warning-tohumanity.html

United Nations Conference on Environment and Development. (1992). Earth Summit '92. London: Regency Press.

United Nations Development Programme. (1994). Human development report: New dimensions of human security. New York: Oxford University Press.

United Nations Development Programme. (1998). Human development report:

Consumption for human development. New York: Oxford University Press.

Upham, P. (2000). An assessment of the natural step theory of sustainability. Journal of Cleaner Production, 8, 445.-454.

Uzzell, D., Pol, E., \& Badenas, D. (2002). Place identification, social cohesion, and environmental sustainability. Environment and Behavior, 34, 26-53.

Venetoulis, J., Chazan, D., \& Gaudet, C. (2004). Ecological footprint of nations. Oakland, CA: Redefining progress. Retrieved 3/16/07, from http://www.rprogress.org/newpubs/2004/footprintnations2004.pdf

Vickers, V. \& Mathews, C. (2002). Children and place. Science Activities, 39(1), 16-25.

Vitek, W. \& Jackson, W. (Eds.) (1996). Rooted in the Land: Essays on Community and Place. New Haven, U.S.A.: Yale University Press.

Vörösmarty, C. J., Lévêque, C., Revenga, C., Bos, R., Caudill, C., \& Chilton, J. (2005). Fresh water. In R. Hassan, R. Scholes \& N. Ash (Eds.), Ecosystems and human well-being: Current state and trends: Findings of the Condition and Trends Working Group of the Millennium Ecosystem Assessment (pp. 179). Washington: Island Press. 
Welsh, H. H. (1994). Bioregions: An ecological and evolutionary perspective and a proposal for California. California Fish and Game, 80(3), 97-124.

Wheatley, M. A. (1997). Social and cultural impacts of mercury pollution on aboriginal peoples in canada. Water, Air and Soil Pollution, (97), 85-90.

White, C., Woodfield, K., \& Ritchie, J. (2003). Reporting and presenting qualitative data. In J. Ritchie \& J. Lewis (Ed.), Qualitative research practice: A guide for social science students and researchers (pp. 287-320). London: Sage.

Wilkinson, C. (2005). Blood struggles: The rise of modern Indian nations. New York: W.W. Norton \& Company.

Winter, D. (1996). Ecological psychology: Healing the split between planet and self. New York: Harper Collins College Publishers.

Witherspoon, G. (1977). Language and art in the Navajo universe. Ann Arbor: University of Michigan Press.

Wolf, E. C., \& Zuckerman, S. (Eds.). (2003). Salmon Nation: People, fish, and our common home ( $2^{\text {nd }}$ ed.). Portland, OR: Ecotrust.

Woody, E. (2003). Recalling Celilo. In E. C. Wolf, \& S. Zuckerman (Eds.), Salmon Nation: People and fish at the edge ( $2^{\text {nd }}$ ed., pp. 9-16). Portland, OR: Ecotrust.

World Commission on Environment and Development. (1987). Our common future: Report of the World Commission on Environment and Development. United Nations Environment Program. Oxford: Oxford University Press.

World Wildlife Fund (2004). Living planet report. Gland, Switzerland: World Wide Fund for Nature.

Zucker, J., Hummel, K., \& Hogfoss, B. (1983). Oregon Indians: Culture, history \& current affairs, an atlas \& introduction. Portland, OR: Western Imprints, the Press of the Oregon Historical Society.

Zwick, M. Elements and relations: Aspects of scientific metaphysics. Unpublished manuscript. 


\section{APPENDICES}

Appendix A: Interview script

Welcome \& Introduction

Thank you, __ (name), for taking the time to join me today and share some of your knowledge and perspective. As you know from our previous communications, this project is about exploring the perspectives of indigenous leaders on the topic of sustainability. Our aim today is to hear from you on the subject. Shall we begin?

\section{Personal Orientation (5-10 minutes)}

o Please share a bit about yourself: your heritage; where is home for you; the focus of your work?

o How would you describe your own cultural identity? How does your identity relate to your life's work?

o Later I will ask questions about "your community.” How would you define your community in terms of geography and population of people? In other words, what places and people make up your community?

\section{Sustainability as a Concept (20-40 minutes)}

o The term "sustainability" is being used by many people who are seeking to address the social and environmental challenges of today. Is "sustainability" a term that you use or is there another term you prefer to use?

o What comes to mind when you think of "sustainability" (or alternative term)? How do you define/explain the term?

o Is there a similar term to sustainability in your Native language? (If yes) How is it used in similar or different ways?

o What brief stories, analogies ${ }^{5}$, and/or metaphors ${ }^{6}$ do you use, if any, to convey the idea of sustainability?

o Are there any images or symbols that come to mind that represent the concept of sustainability in some way. (If yes) Could you draw a sketch of the image and tell me about its meaning? (If no) That's fine, I may return to this question at the end of the interview to see if any imagery has come to mind. ${ }^{7}$

-5 minute break-

\section{Vision of a Sustainable Future (60-80 minutes)}

Earlier you identified your community as (their earlier response paraphrased). I would like you to allow your imagination to take you into the future. Imagine a future, in an ideal sense, where your notion of sustainability has taken form in your community.

\footnotetext{
${ }^{4}$ If an alternative term has been suggested, all subsequent uses of "sustainability" in the interview script will be substituted with this alternative term.

${ }^{5}$ If asked to clarify, an analogy will be explained to mean meaningful comparing the features of two things to suggest their similarity.

${ }^{6}$ If asked to clarify, a metaphor will be explained to mean using a word or phrase that usually indicates one thing to indicate another; drawing an implied comparison between the two.

${ }^{7}$ A second inquiry about an image or symbol will come at the very end of the "Vision" section.
} 
You are there as an observer; take a minute to bring this vision into greater clarity. In responding to the following questions I would like you to remain as an observer of this future place and report to me what you see. Whenever you're ready, please begin to share what you see in your vision of a sustainable future.

"Artifacts"

\section{Relationships}

o Please describe the nature of your community's relationship with the environment?

o Describe the nature of the relationships within your community?

o Describe relationships with external organizations, groups and/or institutions?

o What's the nature of your community's relationship with the scientific community?

o Where do the tensions/ conflicts arise among any of these relationships? MisC

o What institutional capacities are in place within your community?

o What role(s) do new technologies play?

o What role(s) do traditional practices play?

o Is there any conflict or tension between the new technologies \& traditional practices?

o What do you not see in this vision of a sustainable future? ${ }^{8}$

"Worldviews"

o Let us now consider the worldviews held by the members of this community. What are the people's values ${ }^{9}$ and core beliefs about reality?

o Please speak to how at least one of these values or beliefs directs people's behaviors towards sustaining community and environment.

0 Imagining yourself as an average person living in the time and place of your vision of a sustainable future. Ask yourself, "who am I?” In doing so, complete the "I am ___ statements on this sheet ( I I am" form provided)

o What does your list of "I am" statements say about your identity/sense of self as this future person?

o Before moving on, what other aspects about your vision of the future would you like to share?

o Please allow yourself to return to the present moment.

\section{-5-10 minute break-}

\section{From Here to There (20-30 minutes)}

With this vision of a sustainable future fresh in mind, I would like to know your thoughts on the necessary actions and strategies needed to begin/continue from this day forward to bring this vision into reality. We will consider these questions from today's context and view the path ahead-how to move along a path towards a sustainable future.

o What actions and strategies will it take to make this vision of sustainability a reality? (Probe to identify actions at the individual, community, tribal, indigenous, national, international, global levels.)

\footnotetext{
${ }^{8}$ Question developed based upon recommendation from Smith \& Anderson (2001).

${ }^{9}$ Values refers to ideals held that imply a preference for certain actions over others
} 
o Which of these actions or strategies do you see as standing out as a key driving force?

o What are the most important obstacles to be confronted?

o Realistically speaking, how many years do you believe it will take to bring this vision into reality?

o Before closing, I would like to ask whether there is anything you would like to add to our conversation today? What of relevance have we not yet touched on?

o In closing what ideas/points from our conversation would you like to emphasize?

I'd like to thank you for the time you've taken today to share your knowledge and perspective. It has been a pleasure and an honor. In the next couple weeks we will send you a transcript of this conversation and invite you to review it for accuracy and you are welcome to make any additions or corrections that you see fit. From there we will remain in touch as the project progresses. Thanks again, and best of luck to you in your work.

— conclude interview (estimated total of 2-3 hours) - 
Appendix B: Disclosure of researcher's relationship with Ecotrust

This appendix is included to provide full disclosure of my relationship with Ecotrust, the non-profit organization the project is being conducted in collaboration with. Ecotrust, headquartered in Portland, OR, is a conservation organization committed to building Salmon Nation, a place where people and salmon thrive (see www.ecotrust.org/about for more information about the organization and its mission).

My connection with Ecotrust formed through two interacting streams; one personal, and the other a general alignment with my educational and professional aspirations. I came to know several members of Ecotrust's staff through a community game of Ultimate Frisbee played regularly on the Portland State University (PSU) campus during the lunch hour. I was early in my graduate studies and had already taken a serious interest in issues of sustainability. I had encountered some of the work produced by Ecotrust through various workshops, presentations and their public communications. I was impressed by their work and I was attracted to the general philosophy guiding their efforts towards resolving contemporary environmental, social and economic challenges.

In the summer of 2004 I enrolled for a graduate course: Organizational Theory and Systems Dynamics collaboratively taught by Wayne Wakeland, Ph.D. and Bob Sinclair, Ph.D., professors in the Systems Science and Psychology departments, respectively. A cornerstone requirement of the course was to conduct a consulting project with a local organization. I approached several of the Ecotrust staff I had become acquaintances with about the possibility of working with them. Their interest and our exploration of the possible topics led to a project that looked at some changes being undertaken in Ecotrust's organizational structure and the accompanying redefined roles and communication channels. We sought to provide a reflection of where they were at, where they were headed, and our thoughts on whether the new structure was likely to take them in their desired direction. Our work was well received. The project also provided me with an intimate look at the organization through the eyes of its employees, and served to deepen my level of respect for their work, guiding philosophy, and overall mission.

After completion of that project, I maintained loose communication with members of Ecotrust's staff to keep open the possibility of further collaboration. A couple years passed, I completed my comprehensive exams, and I began to seriously consider topics for my dissertation. I had identified an interest in working in some capacity on Native issues and sustainability. I informed Craig Jacobson, Ecotrust's Director of Native Programs, of my interest and began to explore possible topics that would fulfill my requirements for my dissertation, align with the work that Ecotrust was engaged in, and have the potential for contributing to meaningful change. We had a series of conversations, I generated several outlines of project ideas, and Craig shared those with his colleagues. This process identified the idea of exploring aspects shared thinking among indigenous leaders within the bioregion on the subject of sustainability as a worthy project to pursue.

Since identifying the general project idea, communication about the project specifics has been maintained with Craig throughout the proposal development phase. Craig has also provided feedback on numerous occasions about cultural appropriateness 
of the recruitment strategy and content of the interview questions. Additionally, Ecotrust contracted me to conduct a series of interviews focused within the Copper River region of Alaska, and compose a report based on those interviews. The content of these interviews were included as part of this larger project. Interest remains to integrate the results produced here with Ecotrust's other programmatic efforts. This project was pursued with the dual intent of fulfilling the requirements for a Ph.D. at PSU, and contributing to the organizational mission of Ecotrust of building Salmon Nation. 
Appendix C: Initial project summary document. Was shared with prospective participants during subject recruitment phase and other interested parties.

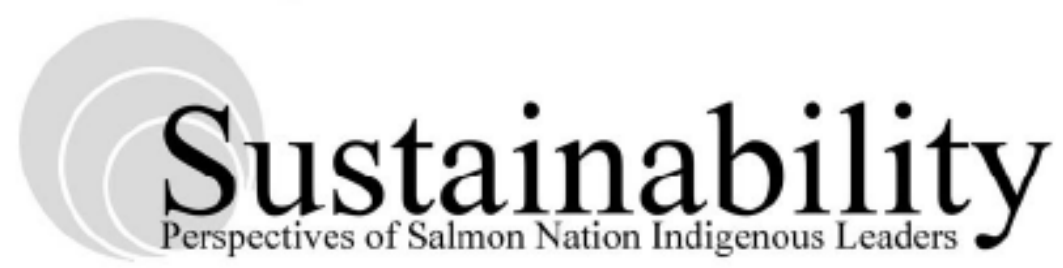

\section{Project Summary}

Extensive research suggests that collective human behavior is on an unsustainable path. In an effort to facilitate change, this project entails conducting in-depth interviews with respected Native leaders across the bioregion of the Pacific Salmon runs. These interviews will explore:

- the meaning of "sustainability"

- visions of what a sustainable future would look like

- actions that must be taken to achieve such a future

The perspective of these leaders may provide valuable insight and inspiration towards shaping a sustainable future for the bioregion and beyond. As such, effort will be made to communicate the results to reach Native and non-Native audiences alike. Products of the project will include:

- Narrative report summarizing the interviews

- Series of concept maps visually illustrating key ideas

- Public presentations

- Interactive web-site

\section{Potential Benefits}

- Identify aspects of shared thinking and language to improve communication about social \& environmental challenges

- Develop a road map for a sustainable future by articulating a clear vision and the actions needed to achieve it

- Enhance awareness and understanding of Native perspectives

- Contribute to local, regional, and global conversations about sustainability

\section{Basic Backoround}

Developed as the dissertation project of David Hall towards completion of a Ph.D. in Systems Science: Psychology at Portland State University. The project is intended to align with the organizational mission of Ecotrust (www.ecotrust.org/about), and is advised by Jan Haaken, Ph.D., the Honorable Elizabeth Furse, Eric Mankowski, Ph.D., Wayne Wakeland, Ph.D., and Virginia Butler, Ph.D.

\section{Contact}

David Hall, M.S.

503-799-5922

deh@pdx.edu 
Appendix D: Participation outline. This document was shared with prospective participants during the recruitment phase.

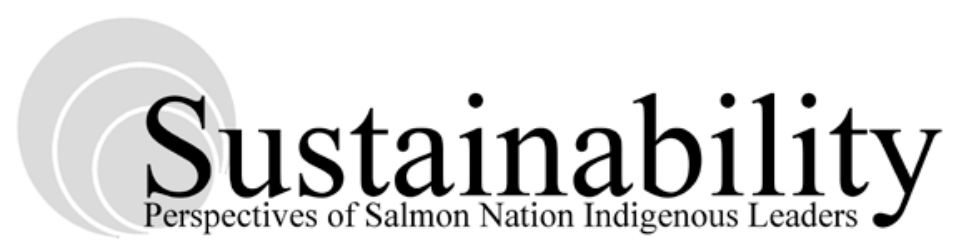

WHAT WOULD PARTICIPATION ENTAIL?

Thank you for your consideration in becoming a participant in this research project. If you decided to take part, your involvement would entail the following:

1) Interview: We will coordinate a time and location that works well with your schedule. The interview itself will take about two hours, with some necessary time at the start to get settled and at the finish to wrap up. The interview would be tape recorded to ensure accuracy, and potentially videotaped with your consent.

2) Review interview transcript: After the interview, a transcript of the conversation will be created and sent to you for your review. You will have about two weeks (or other agreed upon time) to review the transcript and make edits or omit sections as you see appropriate. Once we have received the returned transcript, we will honor your revisions by altering the original records.

3) Evaluate project report: After the all the interviews, a summary report will be composed including a summarization of the perspectives shared. You will be provided with a copy of this report, and a short evaluation form to assess your level of endorsement of the report. Your feedback on the report will be appreciated within two weeks of having received it.

Beyond these things, we welcome more engaged involvement if you have interest and see value in contributing more of your time and effort to advancing the ideas and insights that arise out of this project.

If you have questions, or would like to express your interest in participating, please contact me (David) directly. Also, feel free to direct questions to any of the individuals listed below who are advising the project. Thanks again for your consideration!

David Hall, Ph.D. Candidate, Portland State University 503-799-5922, deh@pdx.edu

Jan Haaken, Professor of Psychology at Portland State University, Committee Chair 503-725-3967, haaken@aracnet.com

The Honorable Elizabeth Furse, Director of the Institute for Tribal Governance 503-725-6426, fursee@pdx.edu

Craig Jacobson, Director of Native Programs, Ecotrust 503-467-0773, craig@ecotrust.org 
Appendix E: Letter of informed consent. Shared with prospective participants during recruitment phase and signed at time immediately before actual interviews took place.

\section{LETTER OF INFORMED CONSENT}

Dear Prospective Research Participant,

You are invited to participate in a study that seeks your perspective as a community leader on the subject of sustainability. Participation will entail an interview asking questions about the meaning of sustainability from your perspective and your vision of a sustainable future for your community. You will also be asked your thoughts on the actions needed for creating a sustainable future. The interviews will provide the information for my doctoral dissertation as a student of psychology and systems science at Portland State University.

The purpose of this research is to identify areas of shared thinking around the idea of sustainability among respected indigenous leaders throughout the bioregion defined by where the Pacific salmon run. To the extent that there is shared thinking, this can provide valuable guidance towards effective communications and collaborations towards sustainability. With your input, the project also seeks to articulate a positive vision for the future for both native and non-native audiences. In general, the perspectives offered in the interviews are anticipated to provide valuable contributions to the regional and global conversations about sustainability.

It is important to note that the interview will be audio recorded for accurate documentation and possible inclusion in public education efforts. As a precaution, you may not want to discuss sensitive community or tribal information. As a safeguard, you will be given an opportunity to review a transcript of the interview and you may exclude content from the project if you so desire. Portions of the transcript you request for exclusion will be deleted from both the written and taped records. Interview content you do not exclude may become part of communication efforts to educate and positively influence thinking on the subject of sustainability. Communications may be in print, public presentations, on-line, or other media productions. You will be informed of any such productions, and any materials developed will be shared with you.

Your participation is entirely voluntary. If you decide not to participate in this study it will not adversely affect your relationship with anyone in the Portland State University community or elsewhere. If you choose to participate, you may withdraw your consent by exiting the interview at any time. If you choose to participate, the interview will be a one-time event that should take roughly 2-3 hours to complete. During the interview we will take breaks as frequently as needed. The interview may cover topics of personal significance. If you become emotionally uncomfortable you are encouraged to request a break at any time or exercise your right to discontinue the interview.

Please feel free to ask me if you have any questions about the study and what your participation will entail. If you have concerns or problems regarding your participation in this study, or your rights as a research participant, please contact the Human Subjects Research Review Committee, Office of Research and Sponsored Projects, 111 Cramer 
Hall, Portland State University, 1-877-480-4400. You may also directly contact my advisor, Jan Haaken, Ph.D. (503-725-3967, haakenj@pdx.edu). You will receive a copy of this letter for your records.

Thank you for giving consideration to participating in this project.

Sincerely,

David E. Hall, M.S.

Department of Psychology

Portland State University

P.O. Box 751

Portland, OR 97207

deh@pdx.edu

503-799-5922

I, (please print name), have read the above letter of informed consent and hereby volunteer my participation.

Signature Date

Please check one:

I am okay with quotes from the interview having my name associated with them.

$\square$ I prefer to have any quotes from the interview be presented confidentially (by checking this box I am also ensuring that the audio-recordings will be used exclusively for accuracy of the research). 
Appendix F: Videotaping letter of informed consent. Shared with prospective participants during recruitment phase and signed at time immediately before actual interviews took place.

\section{LETTER OF INFORMED CONSENT FOR VIDEOTAPING THE INTERVIEW}

I, , have already read and signed the letter of informed

consent. As such, I am well informed about the nature of the study, the role of my participation and possible risks and benefits of participation. The researcher has offered to answer any questions I have about the study.

I understand that there is an option of having my interview videotaped. The videotapes will serve as a back-up recording to the audio-tapes to ensure accuracy in the documentation of my comments shared in the interview; and be used for inclusion in public education efforts through a website and possible documentary productions.

I understand that I will be given an opportunity to censure any of my comments or segments of the videotape that I prefer to not be made publicly available. I realize that the portions I do not censure may be viewed for the purposes of working towards completion of his dissertation project, public presentation of the research results, and public education through the internet and potentially other media outlets.

I have read the above information, and I hereby authorize the researcher to videotape the interview and use my words, image and/or voice in his study and for public education.

Signature Date

If you have questions about this study, please contact David Hall (503-799-5922 or deh@pdx.edu), his advisor, Jan Haaken, Ph.D. (503-725-3967, haakenj@pdx.edu). If you have questions or concerns regarding your participation in the study, please contact the Chair of the Human Subjects Committee at Portland State University, 111 Cramer Hall (1-877-480-4400). 
Appendix G: “I am” statements

Below is the complete list of "I am" statements generated by participants in response to the following question: "Imagining yourself as an average person living in the time and place of your vision of a sustainable future. Ask yourself, 'who am I?' In doing so, complete some 'I am___ statements on this sheet.” Due to time constraints, not all participants were asked this question, and due to the physical set-up of a given interview, participants may not have been requested to write their responses on a sheet of paper.

The "I am" statements are organized here in accordance with the codes ascribed to them via the framework and process outlined in the Methods chapter. Numerous statements proved difficult to categorize, and the researcher appreciates the sentiment that such categorization reduces and distorts the meaning. To minimize this effect, it is recognized that these statements provide a classic example of the whole being greater than the sum of the parts. One's identity can never be fully expressed through words, let alone singular statements, but in viewing the gestalt of these statements some appreciation can be gained for the general nature of identity collectively envisioned by the participants.

Definitions of code categories:

- Universal / Transcendent

- Statements that speak to identity as characterized by a state of being that is indefinable, beyond categorization

- Individual attributes

- Statements that emphasize identity in terms of individual-level attributes

- Individual states of mind

- $\quad$ Statements that speak to individual attitudes and states of mind, or subjective experience

- Individual/Social affiliation

- Statements that speak to an individual's interactions with others

- Social affiliation

- Statements that emphasize identity as connected with a social group

- Social/Ecological affiliation

- Statements that emphasize identity as connected with a social group and imply a particular place or geographic region

- Ecological affiliation

- Statements that emphasize identity as connected with ecology and the land

Universal / transcendent:

- I am that I am...I'm defining myself in an un-definable way that has no limit. (LM).

Individual attributes:

- I am a person like anybody else and I have something to say (AM).

- I am smart (AM, JH). 
- I am a strong person (AM, CC).

- $\quad$ I am persistent (AM).

- I am eloquent (AM).

- $\quad$ I am brave (AM).

- I am beautiful (JH).

- I am fruitful (JH).

- I am it. I get it (JH).

- I am a woman (NM).

- I am growing old. Some people think that's a bad thing (SY).

- I am long-lived. I want to be here for a while (SY).

Individual states of mind:

- I am thankful. Thankful for the gifts and opportunities that were given to me, and for the ones that got me here (SY).

- I am respectful. I am respectful for those gifts that were given not to be used or taken advantage of (SY).

- I am happy (KM, SY).

- I am proud of who I am (CC).

- I hopeful for tomorrow (JH).

- I am still hopeful that we will have a sustainable future (CC).

- I am hoping for the best for the future generations (CC).

Individual/Social:

- I am considerate (JH).

- I am a compassionate woman (CC).

- I am fluent in my language (NJ).

- $\quad$ I am speaking my language (KM).

- I am an advocate for my people (NM).

- I am a supporter of other communities (NM).

- I am respected (KM).

- I am liked (JH).

- I am giving more than I am taking (NM).

- I am willing to share (SY).

- I am sharing all I know and understand about who I am, and who we are as Yakama people (CC).

- I am committed to educating people about us, and who we are so they will understand (CC).

Social affiliation:

- I am a member of a community (NM).

- I am responsible for people (BC).

- I am responsible for young lives and old lives (BC).

- I am an aunt (BC).

- I am a sister (BC).

- I am a daughter (BC).

- $\quad$ I am a provider (KM). 
- I am a teacher (KM).

- $\quad$ I am a student (KM).

- $\quad$ I am an elder (KM).

- $\quad$ I am a wife (KM).

- I am a mother (KM).

- I am part of the seventh generation since the signing of the 1855 treaty (CC).

- I am Irish too! (NM).

- I am multicultural (NM).

Social/Ecological affiliation:

- I am Native (KM).

- I am Indian. I am Athabascan, from Alaska, Mentasta area (JH).

- I am Yakama (CC).

- I am Alaskan Native (NJ).

- I am a Native American (NM).

- I am well knowledgeable in my traditional and cultural ways (NJ).

- I am living my traditional culture (KM).

Ecological affiliation:

- I am responsible for a landscape that sustains me (BC).

- I am treading lightly on my path (NM).

- I am a consumer (BC).

- I am from this land, I belong to this land, I will never be from anywhere else. I am here forever $(\mathrm{BC})$.

- $\quad$ I am home (JH).

- $\quad$ I am dependent on the fish and moose and so forth (JH).

- I am eating my traditional food (KM).

- I'm healthy (JH, KM, NJ, SY). **

** "I am healthy" was a particularly difficult item to code using this coding scheme. Ecological affiliation was chosen based on several other statements that spoke to health as being inherently linked with a healthy environment. For example: "You have to have a healthy landscape to have healthy food in order for us to be healthy" (CC, P 196). 
Appendix H: Field notebook sample entry. Entry composed at end of June 2007 Alaska visit after having conducted the first five interviews of the project.

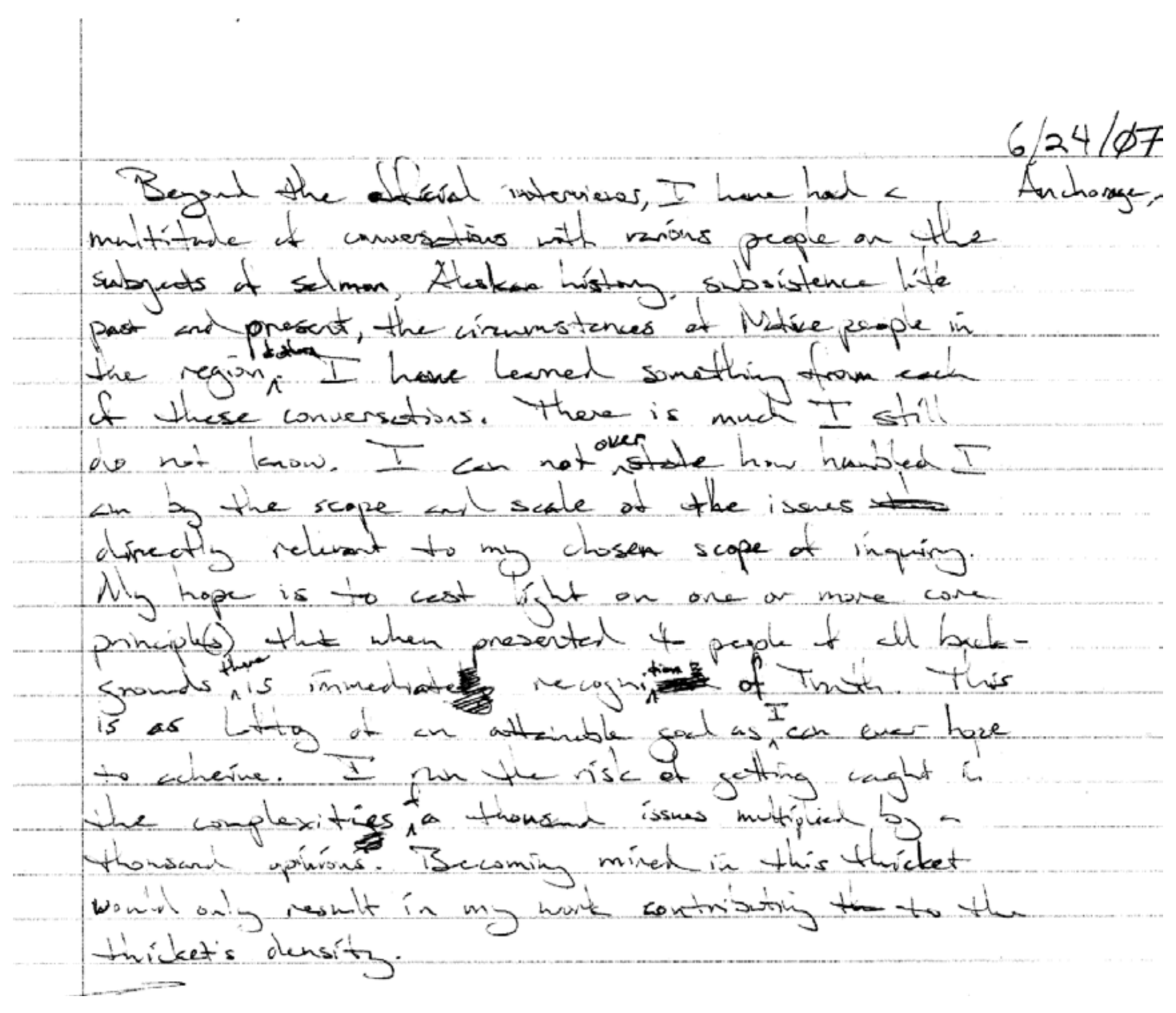


Appendix I: Testimonial validity survey data

Survey administered on-line via Vovivi EFM Continuum.

1) Responses Received: 10

\begin{tabular}{|l|l|}
\hline Antone Minthorn & Kathryn Martin \\
\hline Bobbie Conner & Larry Merculieff \\
\hline Carol Craig & Nichole Maher \\
\hline Dennis Martinez & Shawn Yanity \\
\hline J oeneal Hicks & Wilson J ustin \\
\hline
\end{tabular}

2) Which of the following statements best reflects your position?

\begin{tabular}{|c|c|}
\hline Name & $\begin{array}{l}\text { Response option selected to question: } \\
\text { "Which of the following statements best reflects your position?" ** }\end{array}$ \\
\hline Antone Minthorn & "I am comfortable with my name being associated with this narrative as it is." \\
\hline Bobbie Conner & "I am comfortable with my name being associated with this narrative as it is." \\
\hline Carol Craig & "I am comfortable with my name being associated with this narrative as it is." \\
\hline Dennis Martinez & "I am comfortable with my name being associated with this narrative as it is." \\
\hline Jeannette Armstrong & $\begin{array}{l}\text { E-mail correspondence: "I was fine in general with the } \\
\text { statements. Good work." (personal communication, April 30, 2008) }\end{array}$ \\
\hline Joeneal Hicks & "I am comfortable with my name being associated with this narrative as it is." \\
\hline Kathryn Martin & "I am comfortable with my name being associated with this narrative as it is." \\
\hline Larry Merculieff & $\begin{array}{l}\text { "It would require significant changes for me to be comfortable with my name } \\
\text { being associated with this narrative." }\end{array}$ \\
\hline Nichole Maher & "I am comfortable with my name being associated with this narrative as it is." \\
\hline Shawn Yanity & "I am comfortable with my name being associated with this narrative as it is." \\
\hline Wilson J ustin & $\begin{array}{l}\text { "With a few minor changes, I would be comfortable with my name being } \\
\text { associated with this narrative." }\end{array}$ \\
\hline
\end{tabular}

** Response options not selected by any participants:

a) "I am not comfortable with my name being associated with this narrative."

b) "Other (please specify)" 
3) The final thought in the narrative reads: "Gifted with this potential, our purpose is to continuously maintain ourselves and all our relations in harmony and balance with natural law. The extent to which our cultures serve this purpose is the extent to which they are ultimately valid and worthy of being passed along to future generations." Please indicate the you level of agreement with this statement by selecting one of the following:

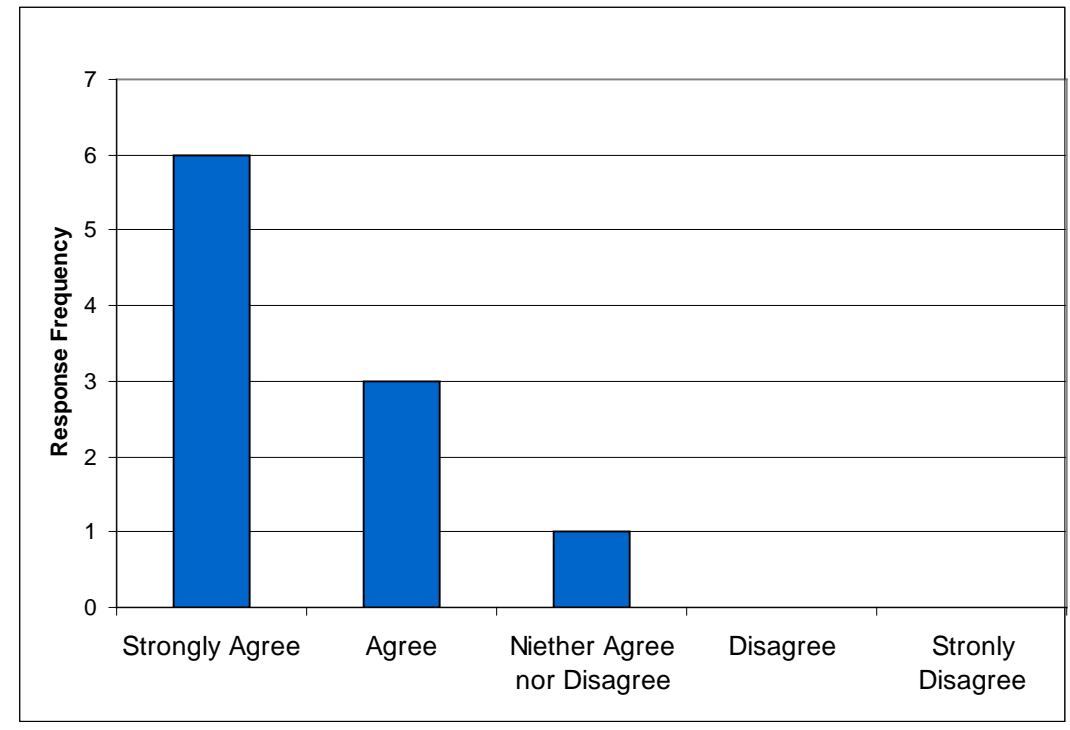

\section{Comment Responses:}

Recognizing God for giving us this gift. 
4) Please rate your level of agreement with the following statements:

4.1) My perspective is represented in this narrative. (Strongly agree $=0$; Strongly disagree $=5$ )

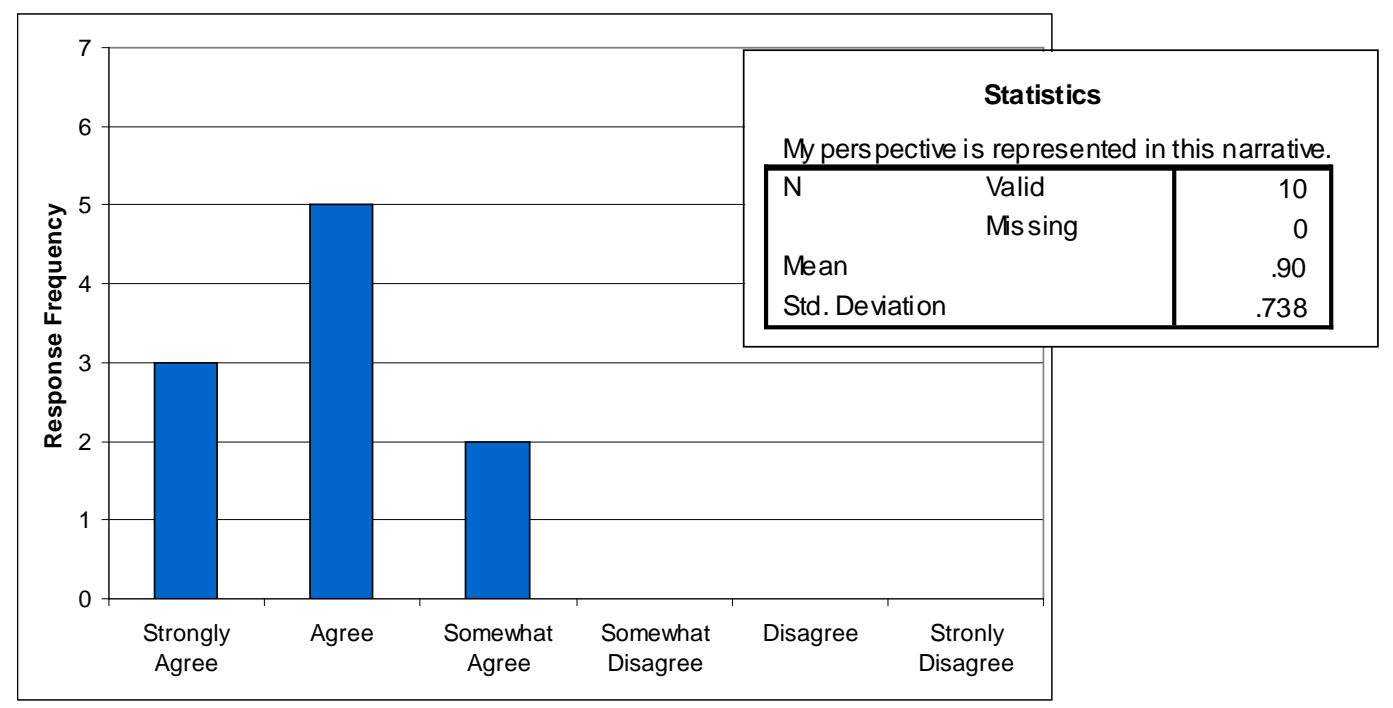

4.2) The key aspects of my thinking on sustainability are included in this narrative. (Strongly agree $=0$; Strongly disagree $=5$ )

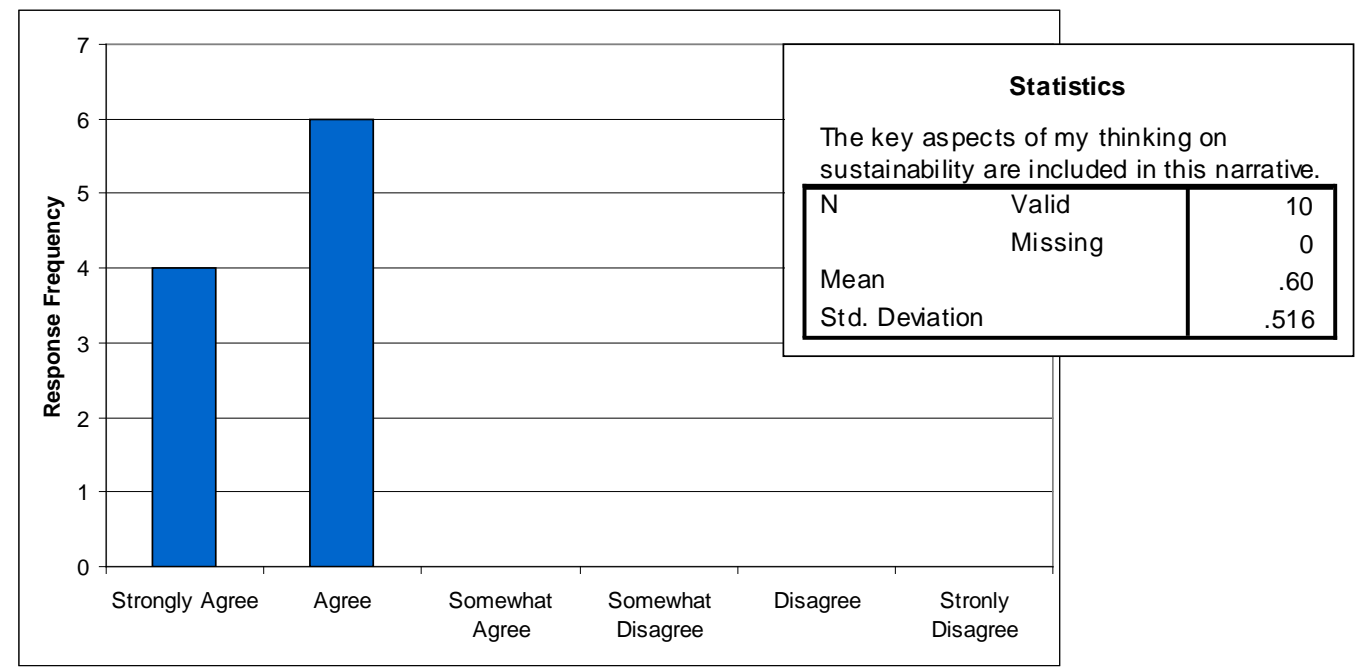


4.3) As a whole, this narrative reflects my thoughts on the subject of sustainability. (Strongly agree $=0$; Strongly disagree $=5$ )

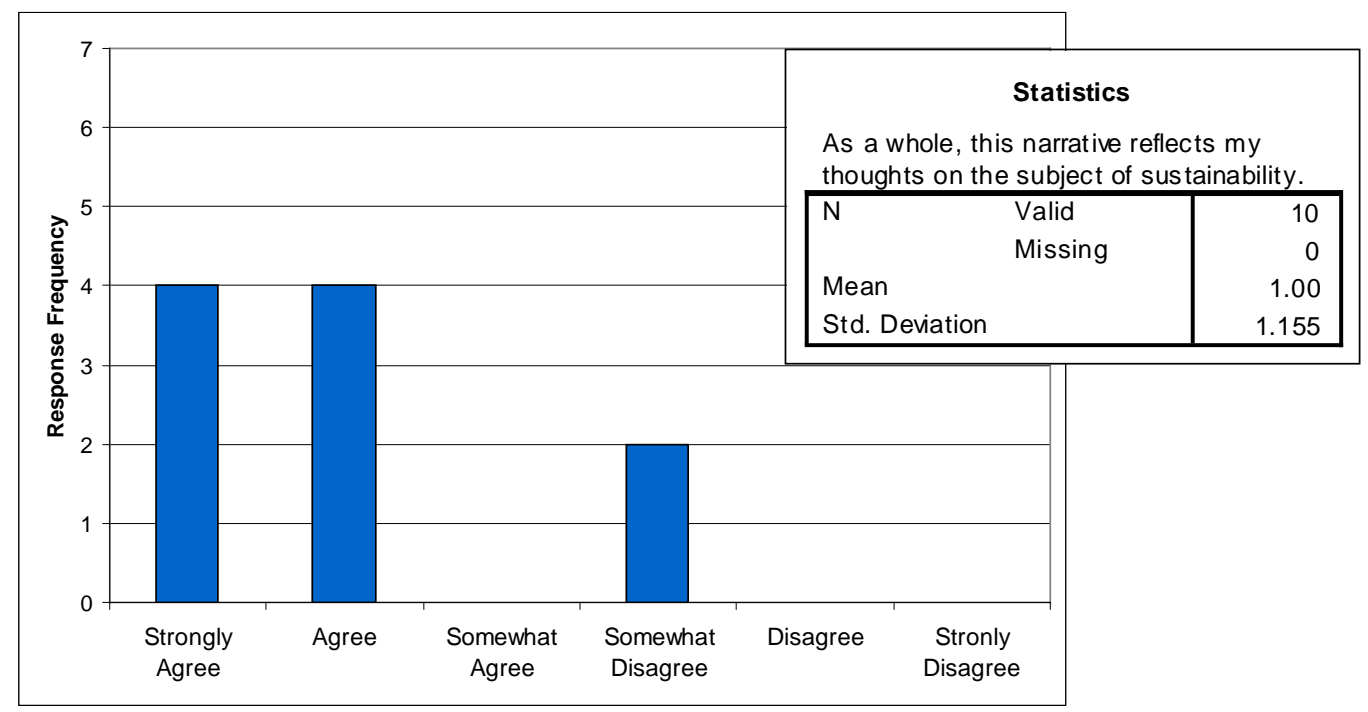

4.4) I would feel comfortable presenting this narrative to my peers as reflective of my perspective. (Strongly agree $=0$; Strongly disagree $=5$ )

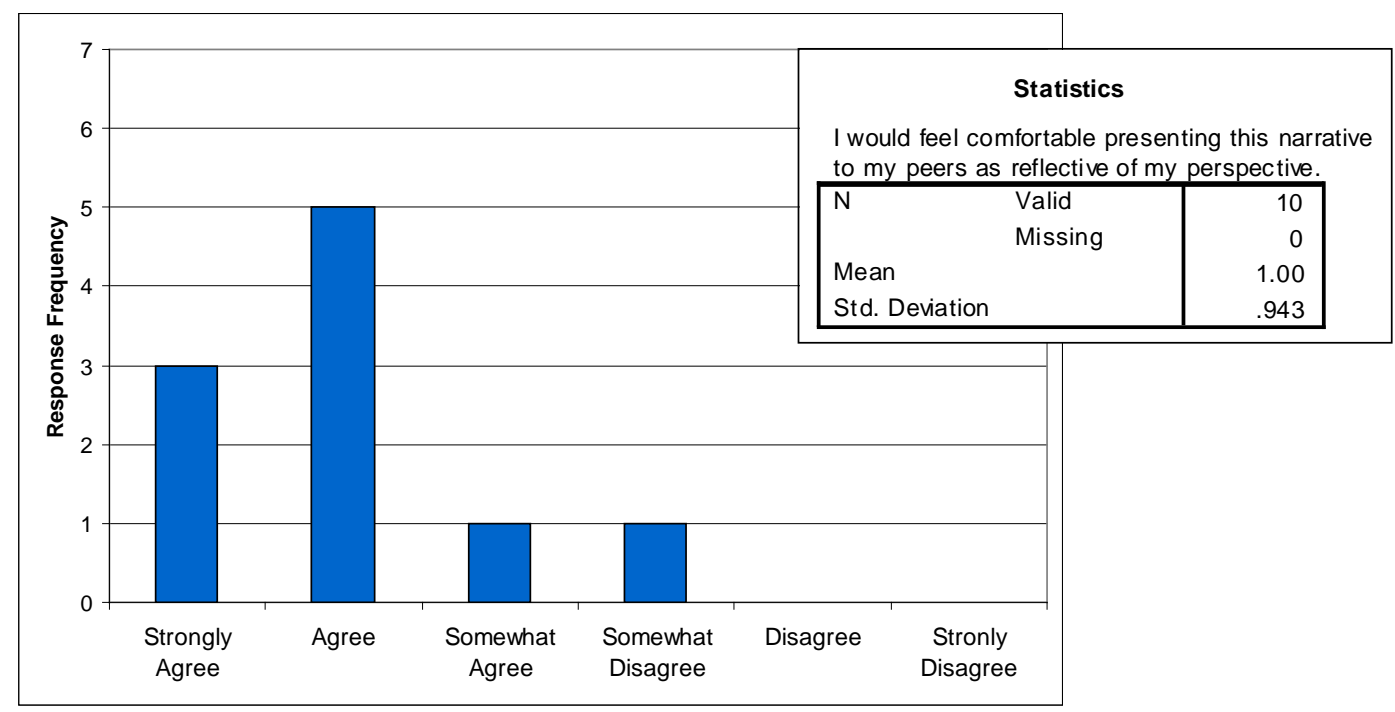

\section{Comment Responses:}

Leadership as a key element in Sustainability seems to be lacking as a subject matter 
5) Did your interview number appear in association with any thoughts that do not accurately represent your perspective?

No: 9

Yes, at least one statement: 0

Yes, multiple statements: 1 (Larry Merculieff)

6) If "yes" to the previous question, please indicate which statements are at fault. If "no," please skip to the next question.

I won't go into it, but basically I have difficulty in my thoughts being part of a collective, even if the collective statement is somewhat accurate or nearly accurate. Indigenous peoples'

statements are frequently taken out of context and the context is important.

7) Were there any statements in the narrative where your interview number was NOT cited, but that you believe you clearly spoke to in your interview and/or are thoughts that you strongly identify with?

No: 8

Yes, at least one statement: 0

Yes, multiple statements: 2

8) If "yes" to the previous question, please indicate which statements you would like to be cited as having spoken about. If "no," please skip to the next question.

Both participants shared via personal communication, and additional citations were added to narrative.

9) Were there any thoughts expressed in the narrative that you strongly disagree with? If yes, please note those thoughts (brief explanations are welcomed but not required).

No, it was well written and with lots of thought given it.

Cultural Sustainability/Humility. "People aren't the most important thing in the universe." My people and my Indian culture are important enough for me to fight you if you threaten me. I suggest no changes. The statement just rankles me.

i don't have the narrative in front of me as I am traveling. There were some I strongly disagreed with....not in the intent expressed, but how it was expressed. For example, I speak to indigenous peoples about not using Western terms like sustainability and natural resources

10) What changes, if any, would you like to make to the narrative?

change all references to natural resources to fish, wildlife, habitat. instead of sustainability, use harmonious relationships or harmony

Troubled by the use of the word "valid" in the final sentence. Would suggest removing that as it is a loaded term, and the meaning of the sentence holds true without it. Need to introduce what 
TEK stands for earlier.

11) What do you like best or find most valuable about this narrative?

it was well written and with lots of thought given it.

It hits home and brings about awareness that even I tend to forget or take for granted. We need to come to the realization that sustainability is about our culture, identity, and tradition. If we want to keep these traits alive, we need to come forward and not just talk the talk, but walk the walk also.

Coherence and accuracy

the diversity of people interviewed with a close perspective on all these ideas.

How this document can be used to educate people on sustainability.

It is a good effort. i understand that the researcher has to develop numerically based data...but in the indigenous worldviews, that is taking information out of context. I think the narrative shows the commonality of thought of the interviewees...but the color of what they say, the flavor if you will, should be kept. Inside the way someone conveys something are jewels of information.

The different views and how they are inter-linked!

The following statements are really important and right on: "We possess greater knowledge than many give us credit for, and with empowerment to design our own economic systems, much can be done to provide for culture and economy and environment." "Considering natural law, the true meaning of sustainability is not theoretical, it is practical." Also, the piece about bridging TEK and Science is really important.

Appreciate the choice to use a collective voice in narrative.

12) Please share any additional thoughts you may have:

Great J ob!

The narrative did not look long enough to the future. Most of what i saw was values as seen, I was hoping a leadership discussion would take place that would synthesize the type of leadership needed to obtain sustainability on a Community basis.

The CTUIR has seen the loss and devastation of their homeland territories. The tribes descended into poverty. They know what that experience is like. What's more, the tribes are not going to leave their reservation homeland but are going to make it stronger to help its people. Therefore, sustainability is a core tribal value. The purpose of our tribal government is to achieve a maximum degree of self governance in all affairs. We walk the talk.

What is the value attributed to the term "Elder?" Is it that an elder is somehow wise and trusted to give advice and information to the community?

Appendix J: Project and narrative critique from Larry Merculieff.

The following e-mail is one of several between Larry Merculieff and David Hall about Mr. Merculieff's concerns with the narrative results as composed. This e-mail speaks further to those concerns documented in the testimonial validity results section and 
Appendix I. This e-mail was composed after an advanced draft of the results section was shared with Mr. Merculieff to inform him of the decisions that had been made in response to his feedback. The e-mail also responds to questions posed that sought to better understand his perspective. It is included here with permission.

Date: $\quad$ Mon, 12 May 2008 11:56:53 -0400 [05/12/2008 08:56:53 AM PDT]

From: "Larry Merculieff"

To: "David E Hall”

Subject: Re: Sustainability project: Narrative results

Hi David. I do appreciate your efforts to open a dialogue. However, it does appear, given your statements in the materials you sent me, that you already have set the way you are going to proceed. As I indicated, I do understand. I had misunderstood your methodology from the very beginning. Had I known you were going to reduce my statements to a consensus statement, I would not have agreed :-) I thought you were seeking different perspectives on the word "sustainability". I believe strongly that indigenous peoples have much to offer the world in different perspectives. It is the existing paradigms in science, economics, and politics that has resulted in the current state of affairs in the world, where all the earth's life support systems are being compromised. Reducing earth to inanimate parts of an organic whole has proven to be anathema in the world. Reducing life to numbers, statements of people to numbers, life to "things", and lumping perspectives of indigenous peoples into statements they never made (and as if they are all a single culture), are parts of systems created by a disconnected consciousness that has wreaked havoc throughout our history.

I am not interested in consensus. I am interested in different perspectives that may contribute to the health and wellbeing of this planet and all who live on it. That, I thought, was the intent of your project. The difference between what Justin and I say and how we say it, for example, may be subtle but profound as each comes a different culture that has something unique to offer the world. A casual observer or someone not versed in cross-cultural subtleties can miss the significance of the slight differences. It is difficult enough that we cannot communicate what and how we think in our own languages and have to use the very limited English language. To reduce what we say even further by creating statements the participant never made makes the exercise pointless in my opinion.

Like I said, I do understand what you have to deal with to make this project pass "muster" and hope you do not take my comments personally. I was a member of the Committee on the Bering Sea Ecosystem under the auspices of the National Research Council, I have presented before the American Assoc. for the Advancement of Science and was one of four Native Americans that presented at the White House Conference on the Oceans, and was a co-principal investigator with a team of world-class scientists from the Shirshov Institute of Oceanology in the Russian Academy of Sciences. I do understand :-)

I give permission for you to use my critique, but would like for you to withdraw references to me in the "consensus" statements you developed :-)

Have a great week.

Larry

Larry Merculieff

Seven Generations Consulting 
Appendix K: Project website. Front page and a participating leader page from www.nativeperspecives.net
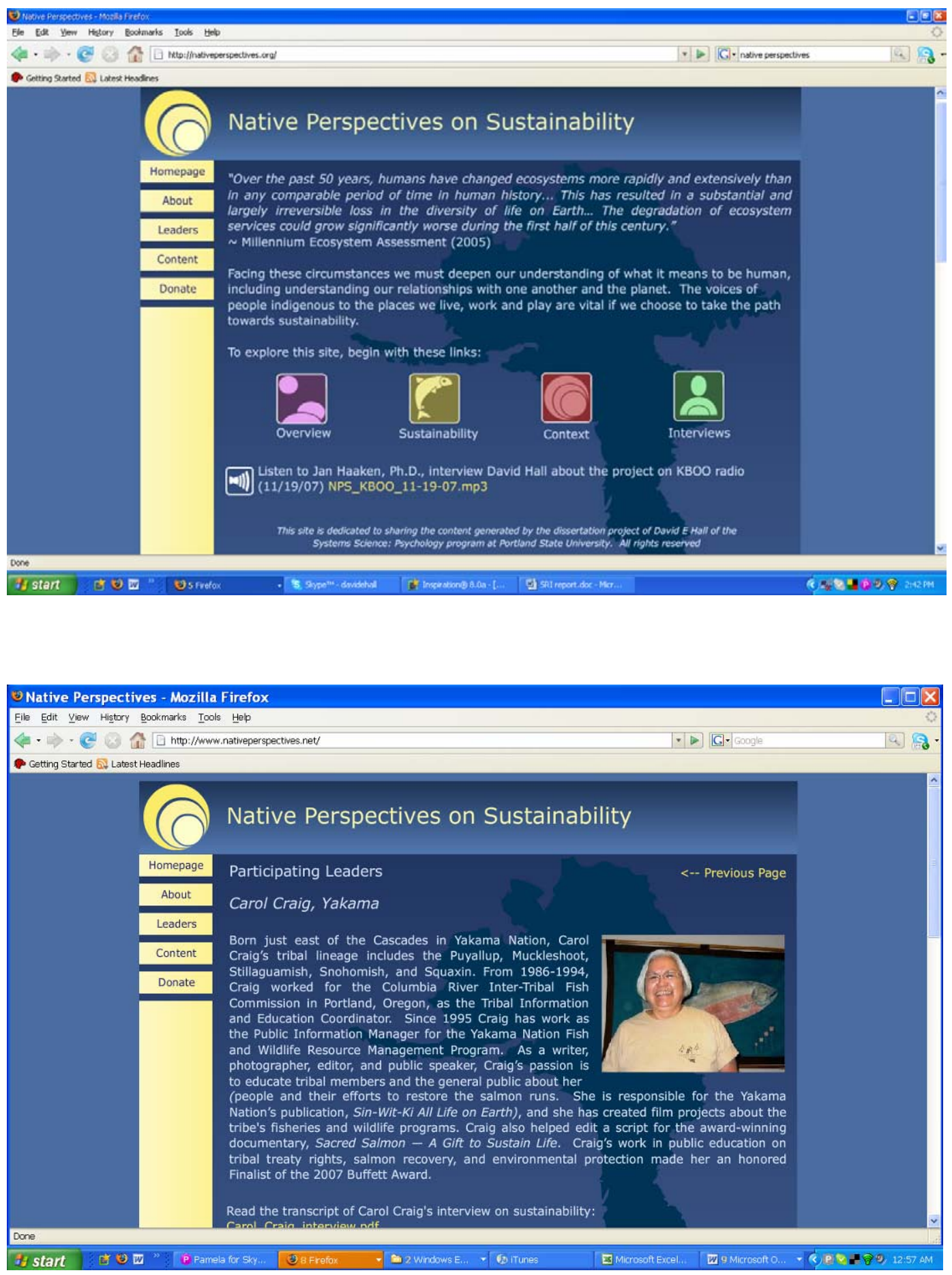
Appendix L: "Native Perspectives on Sustainability" video pieces produced and aired on public access television in the greater Portland area.

As part of the project's commitment to social action and dissemination of obtained information, two videos were produced in partnership with the Flying Focus Video Collective (FFVC) and aired on public access television in the greater Portland area. The pieces were produced by David Hall, with camera work by PC Peri, editing by Caleb Heymann, and airing facilitated by Dan Handelman. "Native Perspectives on Sustainability: Nichole Maher (Tlingit)" (see Figure L1) aired three times during the week of October 29, 2007. "Native Perspectives on Sustainability: Roberta Conner (Cayuse)" (see Figure L2) aired three times during the week of Jan 14, 2008. VHS and DVD copies were also created and made available to the general public through the FFVC catalogue and website. DVD copies were shared with the participants and their respective organizations, the Native American Youth and Family Center (NAYA) and the Tamastslikt Cultural Institute (TCI).

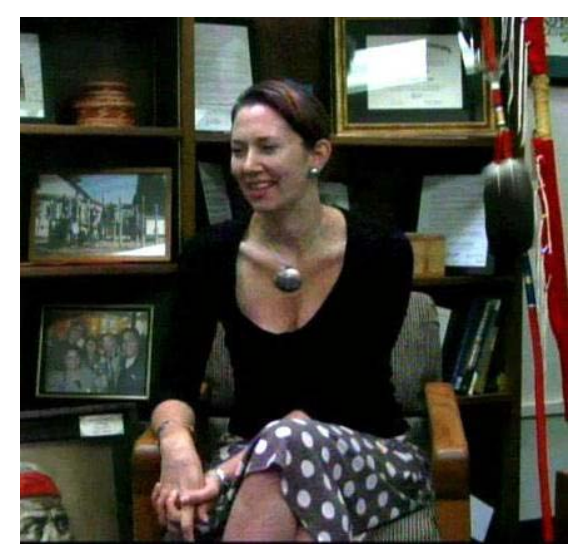

Figure L1. Screen shot from video "Native Perspectives on Sustainability: Nichole Maher (Tlingit)"

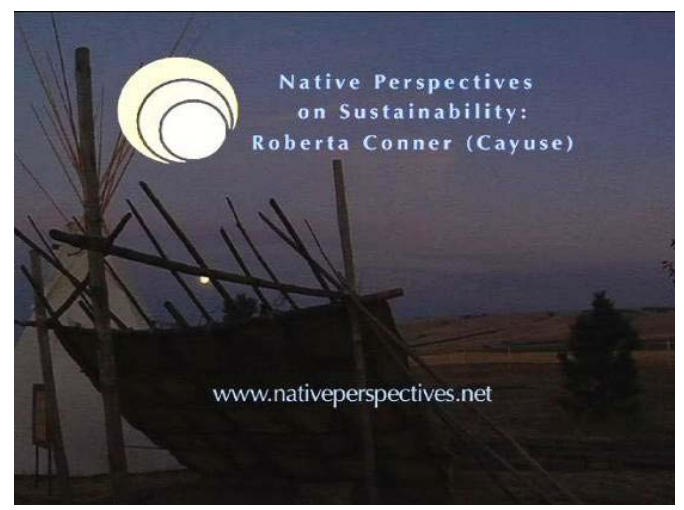

Figure L2. Screen shot from video, "Native Perspectives on Sustainability: Roberta Conner (Cayuse)" 
Appendix M: The naw'qinwixw process of the Syilx people. Quoted and paraphrased from interview with Jeannette Armstrong (pp 6-12).

Syilx, the traditional name the Okanagan people, roughly translates to mean the people with a continuous responsibility to bind with and twine together many strands into one strand. "It's speaking about the ethic of the people, the responsibility or the philosophy of the people, to continuously bind with everything that's around us: our family members, all of our relatives on the land, and continuously maintain one unit. In other words, to be unified, to be in balance, and if we can do that, we can move forward, into the next generation as a whole."

One of the tools used by the Syilx people to achieve that unity and balance is the naw'qinwixw process. It is considered a dialogue tool, a tool for conflict resolution, and a "methodology that can be used for finding out what the best solution to any question might be." The word 'naw'qinwixw' has attached to it a series of symbolic images. "The first part of the word, 'naw'qin,' - ' $a w$ ' has to do with water dripping... really slow, one drop at a time... And 'naw'qin,' the meaning of 'qin' always has to do with the top of the head, or the top of a mountain. So there is water dripping one drop at a time on the top of the head. The last part of the word, 'wixw,' means we do that for each other. I do it for you, you do it for me.” The dripping water is a metaphor, symbolic of knowledge being put into the mind. "If you were to take a drop of water and put it on say cotton, you'd see that the drop slowly permeates the cotton. That action, or that slow infusion into the whole system is what that abstract metaphor is speaking about. If you were to give knowledge in that way, then knowledge becomes integrated into the whole person: into their mind, and their spirit, and their emotions, every part of them. It becomes integrated into their family, and into the work that they do, they way that they live and think. It becomes a part of them.”

In sharing knowledge with one another, we must do it in a way that takes into consideration the feelings, knowledge and background of those we are speaking with (just as you would not speak to a teenager the same way you would speak to your grandmother). The dialogue process requires you to respect the diversity of the community. We can not assume that others know what we know because they have their own experiences. Within the dialogue, your role is to clarify in a way that others can understand what you know, how you feel, how it affects you or things you know about, while also requesting the same be shared with you by others. It is disrespectful and destructive to attempt to convince others of your perspective in an aggressive and dominant way. By approaching with the intention of sharing perspectives to better inform one another, we'll have a better chance at understanding what must be done. It is through diverse points of view that we attain as much clarity as possible, and naw'qinwixw requests that we seek the most diverse view.

"One of our stories teaches us that the most important view is the most minority view, not the majority. And that majority actually is a tyranny; it actually is a violence and a violation of that principal, a violation of us within our differences, within our diversity. In the story it was the smallest bee that was being left out-and I'm not going to go into the full story - but, in the story it's said that the circle couldn't be whole, and they couldn't bring life back to the entity, until the very smallest one, that everyone was excluding, no one wanted around, and no one thought was useful for anything-was just 
a pest. Of course, it was an insect (laughter), and everyone said, 'you don't have anything to offer. What are you going to offer us, you're just a pest?' But, finally, they ran out of options; there was no one there who could resolve the problem, and the little insect came back and said, 'will you give me a try?' And they said 'ok.' So once the insect sang its power song - in the story the insect sang and completed the whole circle. That's what was necessary. Without that small input from that being it wasn't complete. Once that input, the minority person's interests, in other words, were brought into the circle, and everyone included and embraced that minority person, and everything was whole, the last piece was in place. So nothing can be whole unless that last piece is in place, unless the minority voice is there."

When dealing with a problem for which no one person has the answer, we have to have all the factors, all persons in mind. "Because we care about each other, because we're connected: we have to be together, we have to live together, we have to work together, we have to see each other everyday, that has to be the best way to do things. Understanding that, it makes life easy. It makes life secure. It makes life in a community beautiful. It makes life, in a sense, something that you don't want to step out of... But besides the emotional side, the practical side, the practice of that, transforms the community from individuals to a whole healthy organism.”

Another function of the naw'qinwixw process is to achieve the balance between the four polarities that exist in all communities. These polarities include the elders, the innovators, the mothers and the fathers. Collectively, these four polarities "represent the ability for transformative change and/or destruction and/or whole health if they are brought together” (see Figure M1). 


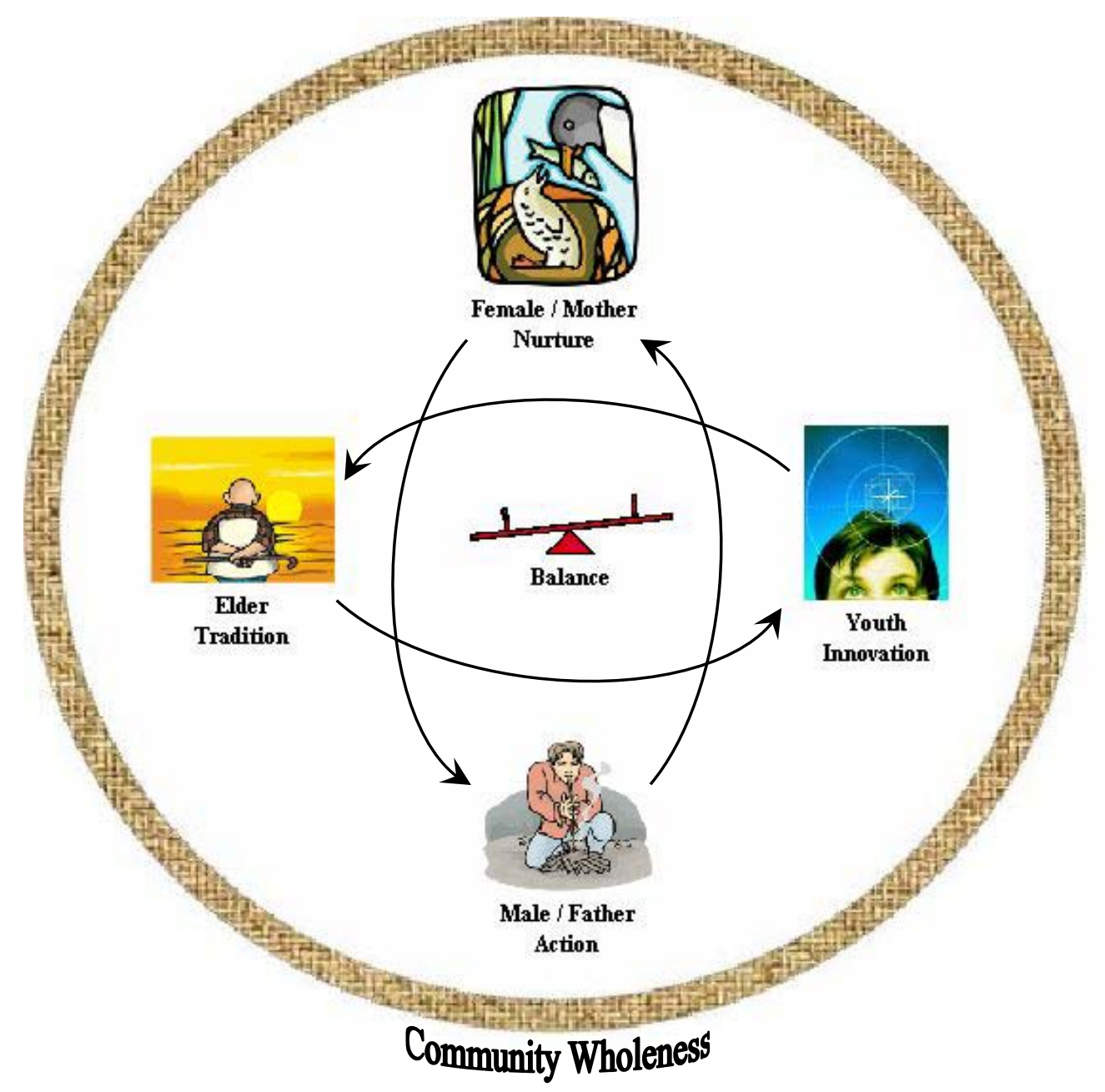

Figure M1. The balancing of polarities through the naw'qinwixw process of the Syilx people. Arrows from one polarity to another indicate the sharing of perspective and knowledge in a respectful manner. The outer circle embodies the weaving or binding together of the community's diversity into one whole, healthy, functioning organism.

The elders, or traditionalists, or conservationist, are those concerned about tradition and history, and hold knowledge about the reasons why things are done the way they are. Their polar opposite is the innovators, the creative people, those who say, 'there are other ways, alternative, new ways of doing things.' In all communities, these two sides will tend to conflict with one another. Without balance, if tradition only had its way, then nothing would change and the community would be unable to adapt to the new challenges it faced. "It would be so rigid it would become brittle and fall apart." On the other hand, if unchecked, innovation and change can go rampant and cause a lot of damage. This is largely what we see today: the greatest creativity and innovation without mediation by tradition and real understanding of the implications.

The other two other polarities are male, or "father," and female, or "mother." These polarities are not the same as gender or as sexuality, as both men and women may be in either category, especially in an egalitarian society. Those who embody the role and the concept of 'mother,' and 'mothering,' understand how to nurture, and how to care 
for others. They understand emotions and attend to the emotional bonding of family and community. While the emotional side of things in a community is extremely critical, if out of balance it can also wreak havoc. The opposite polarity, those who embody 'father,' are those of action and who employ the strengths of the community to build and get things physically done. They are the ones who are able to use logic and analysis and "can pick up a tool and use it" to do what needs to be done. As the opposite polarity of emotion, doers are bothered when people become emotional as it stands in the way of logic, and stands in the way of getting things done. Consequently, they tend to conflict with those who understand emotions and care about the feelings people are experiencing. On their own, the doers can create a lot of havoc, particularly to community relations. When paired with the innovators, the long-term integrity of the community can really be in trouble.

The naw'qinwixw process works to "empower the four to work together, to mediate their polarities and differences, and to bring together their thinking." If we do not balance these polarities, then there will be conflict, dissention, and no solutions that work for everyone. Elders, or innovators, or mothers, or fathers, can still participate within their own group, but everyone must "come back full circle to work with their opposite polarity." Through naw'qinwixw these polarities can converge, converse, and work together towards balance and community wholeness. "It's a governance process that works." 